NBER WORKING PAPER SERIES

\title{
OPTIMAL BANK REGULATION IN THE PRESENCE OF CREDIT AND RUN-RISK
}

\author{
Anil K. Kashyap \\ Dimitrios P. Tsomocos \\ Alexandros P. Vardoulakis \\ Working Paper 26689 \\ http://www.nber.org/papers/w26689
NATIONAL BUREAU OF ECONOMIC RESEARCH
1050 Massachusetts Avenue
Cambridge, MA 02138
January 2020, Revised February 2023

This paper supersedes "How does macroprudential regulation change bank credit supply?" NBER Working Paper No. 20165. We are grateful to Gadi Barlevy, Saki Bigio, Dong Beom Choi, Dean Corbae, John Geanakoplos, Emmanuel Farhi, Todd Keister, Stephen Morris, Enrico Perotti, JeanCharles Rochet, Hyun Shin, Frank Smets, Adi Sunderam, Sergio Vicente, Randy Wright, and especially Harald Uhlig. We also thank the seminar participants at numerous institutions and conferences. Kashyap has received research support from the Initiative on Global Markets at the University of Chicago Booth School of Business, the Houblon Norman George Fellowship Fund, a grant from the Alfred P. Sloan Foundation to the Macro Financial Modeling (MFM) project at the University of Chicago and the National Science Foundation for a grant administered by the National Bureau of Economic Research. Kashyap's disclosures of his outside compensated activities are available on his web page. All errors herein are ours. The views expressed in this paper are those of the authors and do not necessarily represent those of Federal Reserve Board of Governors, anyone in the Federal Reserve System, any of the institutions with which we are affiliated, or the National Bureau of Economic Research.

At least one co-author has disclosed additional relationships of potential relevance for this research. Further information is available online at http://www.nber.org/papers/w26689

NBER working papers are circulated for discussion and comment purposes. They have not been peer-reviewed or been subject to the review by the NBER Board of Directors that accompanies official NBER publications.

(C) 2020 by Anil K. Kashyap, Dimitrios P. Tsomocos, and Alexandros P. Vardoulakis. All rights reserved. Short sections of text, not to exceed two paragraphs, may be quoted without explicit permission provided that full credit, including $(\odot$ notice, is given to the source. 
Optimal Bank Regulation In the Presence of Credit and Run-Risk

Anil K. Kashyap, Dimitrios P. Tsomocos, and Alexandros P. Vardoulakis

NBER Working Paper No. 26689

January 2020, Revised February 2023

JEL No. E44,G01,G21,G28

\section{ABSTRACT}

We modify the Diamond and Dybvig (1983) model so that, besides offering liquidity services to depositors, banks also raise equity funding, make loans that are risky, and can invest in safe, liquid assets. The bank and its borrowers are subject to limited liability. When profitable, banks monitor borrowers to ensure that they repay loans. Depositors may choose to run based on conjectures about the available resources for people withdrawing early and beliefs about banks' monitoring. We model the run decision by solving a novel global game. We find that banks opt for a more deposit-intensive capital structure than a social planner would choose. The privately chosen asset portfolio can be more or less lending-intensive, while the level of lending can also be higher or lower depending on a planner's preferences between liquidity provision and credit extension. To correct these three distortions, a package of three regulations is warranted.

Anil K. Kashyap

Booth School of Business

University of Chicago

5807 S. Woodlawn Avenue

Chicago, IL 60637

and NBER

anil.kashyap@chicagobooth.edu

Dimitrios P. Tsomocos

Said Business School and St. Edmund Hall

University of Oxford

Dimitrios.Tsomocos@sbs.ox.ac.uk

\author{
Alexandros P. Vardoulakis \\ Board of Governors of the Federal Reserve System \\ Constitution Ave NW \&, 20th St NW, Wash 20551 \\ alexandros.vardoulakis@frb.gov
}




\section{Introduction}

It is well understood that financial intermediaries, hereafter banks, provide useful services through both the lending that they undertake and the deposits that they offer. Banks use both sides of their balance sheet to perform these services, which exposes them to credit risk and funding (run) risk. The efficiency of the resulting allocations, from a social point of view, depends jointly on the overall level of lending and the mix of bank assets and liabilities. Yet, most banking models tend to focus on only one service or type of risk, often neglecting how bank fragility endogenously affects the structure of bank balance sheets and vice versa. We propose a new model where banks create economic surplus for both borrowers and depositors but choose a balance sheet that results in excessive run-risk and an inefficient level of lending. We use the model to study alternative regulations that have been proposed since the Global Financial Crisis and derive the optimal regulatory mix.

We start with the setup developed by Diamond and Dybvig (1983), henceforth DD, that explains how a bank can provide liquidity services to depositors (savers) with uncertain consumption/funding needs. We modify DD so that the bank also raises equity, makes loans that are risky, and can invest in safe, liquid assets. We also assume that banks and borrowers are subject to limited liability so that each of them has incentives to take risks that they do not fully bear the costs of. We suppose, similar in spirit to Diamond (1984), that banks monitor the loans to guarantee that they are repaid. Monitoring is costly and, hence, must be profitable for banks to do so. Even if savers were able to monitor borrowers as efficiently as the bank, delegating this function to banks is optimal, because banks can combine monitoring and deposit-granting, which is itself valuable. However, because the loans are risky and have an uncertain liquidation value, depositors may opt to run on the bank depending on the conjectured ability of the bank to serve early withdrawals and the bank's incentives to continue monitoring borrowers. Finally, the bank is managed by a banker who maximizes the profits accruing to her rather than worrying about the utility of depositors or borrowers. The banker internalizes how her choices affect run-risk, deposit supply, and loan demand. Depositors and borrowers rationally expect the equilibrium level of run-risk when making these decisions but are atomistic and do not internalize their marginal impact on the bank's portfolio and run-risk.

We characterize the run decision by introducing a new specification of the type of global game developed by Goldstein and Pauzner (2005) to analyze DD style models. In our setup, depositors get signals about the (liquidation) value of loans that are called early to help pay depositors. Depositors use a threshold rule in deciding to run, such that, if their signal about the liquidation value is below the threshold, they decide to withdraw even if they do not need to consume immediately. Conversely, if the signal is above the threshold, only depositors with urgent consumption needs withdraw, while the rest keep their deposits in the bank. The threshold depends on the deposit contract, the balance sheet, and the profitability of the bank, because the deposit contract specifies the return from waiting instead of withdrawing early, the balance sheet determines the capacity of the bank to serve early withdrawals, and the profitability of the bank governs its incentives to monitor borrowers.

The proof to establish the uniqueness of the threshold is novel and may be of independent interest for other applications of global games. As in Goldstein and Pauzner (2005), there are no global 
strategic complementarities for depositors, but in our setup, the property of state monotonicity is also absent. This property relates to whether the incentive to take an action is monotonic with respect to the underlying fundamentals (state) of the economy-and is typically required in canonical global games (see Morris and Shin, 2003). In our model, state monotonicity would obtain if the relative incentive to run becomes stronger as the liquidation value of loans becomes smaller. If the bank has liquid resources to serve all depositors queuing to withdraw, then state monotonicity holds. But it fails to hold once the bank runs out of liquid resources and, thus, only a percentage of withdrawals can be met. Intuitively, once liquid assets are exhausted, the payoff from withdrawing is higher when the liquidation value of the bank's assets is higher, because the probability of being at a lucky spot in the queue is higher. Hence, state monotonicity is absent.

We employ a new argument to prove uniqueness, which does not require state monotonicity everywhere but only at threshold points. This property is easily satisfied in our framework and we provide intuition about generalizing it to other environments where such perverse state monotonicity obtains. Hence, the proof we propose could be adapted to other applications in which improvements in fundamentals reduce the incentives to act in some regions and increase them in others.

The modifications to DD that we propose are all necessary to assess the differences between the private and social optima. First, micro-founding the probability of a run is crucial to understand the determinants of run-risk. In partial equilibrium, one expects that raising capital requirements or liquidity requirements will reduce the probability of a run. We show this is true in our model. But, in general equilibrium, agents will adjust their behavior in response to regulation, and their new choices will feed back to alter run-risk via equilibrium interest rates.

Second, it is important to explicitly model the optimization behavior of bankers, depositors, and borrowers in order to understand how run-risk affects their choices and welfare. Instead of assuming ad hoc welfare criteria, such as the minimization of run-risk, we model the effect of the choices on agents' utilities. This allows us to compare privately and socially optimal choices. In general they differ, though we also describe a special case where the private equilibrium is constrained efficient.

Third, introducing costly monitoring highlights the importance of the bank's profitability for its stability (over and above the relative liquidity of its assets and the runability of its liabilities). Monitoring is unobservable and costly, so it only occurs if the banker finds it ex post profitable. Policy interventions that crush bank profits can backfire if the banker loses the incentive to monitor. In this case, depositors can opt to run because they realize bank credit risk has endogenously increased because of the lack of monitoring. Hence, we highlight a novel connection between moral hazard and run-risk, which is important for studying regulations that compress banking profits. ${ }^{1}$

Fourth, by modeling the utility impact of agents' choices, it becomes clear that borrowers and depositors care about the amount of lending and the level of deposits that are available. Thus, the overall size of the banking sector matters beyond just the composition of assets and liabilities. The Modigliani-Miller theorem is violated in various ways in our model, but allowing for endogenous

\footnotetext{
${ }^{1}$ This possibility may be of independent interest to researchers as it highlights that runs can occur even for financial institutions that are very liquid, if they lack the incentives to monitor.
} 
equity issuance helps us understand the implications of these violations for the size of the banking sector and how the banking sector converts deposits into loans.

We focus on the normative properties of the model and derive four main findings. First, there are three separate distortions in private banking choices. Both the private and social planner's equilibria can be characterized by the mix of loans versus liquid assets, the mix of deposits versus equity, and the level of lending that is funded by deposits (summarized by the loan-deposit spread). In the private equilibrium, the banker wants to maximize her profits from collecting deposits to extend loans. Because she understands how run-risk and how the profit margin between the loan and deposits rates are each determined, she chooses levels of liquidity and equity that are profit maximizing for her. Compared to the banker, the social planner cares about how these choices affect not only bank profits, but also the utility of depositors and borrowers. We show that the other two agents are disadvantaged in the private equilibrium and explain how the asset mix, liability mix, and the loan-deposit spread can be adjusted to improve the welfare of the savers or borrowers.

Second, each distorted choice by the banker has a component tied to the run and another component that reflects the surplus created by the bank that accrues to either the borrowers or savers. The run component arises because bankers are prone to take risks that raise the probability of a run without internalizing how run-risk directly matters for borrowers' and savers' welfare. This is because the banker is a monopolist and maximizes her own profits subject to limited liability, while borrowers and savers are atomistic, so that they do not internalize how their choices affect run-risk.

Third, how the planner corrects the distortions depends on the weights placed on borrowers versus savers, i.e., on the implicit preference between credit extension and liquidity provision. Runrisk arises from choices on both sides of the bank's balance sheet, and the planner would opt for both a less deposit-intensive capital structure and a less lending-intensive asset allocation to reduce run-risk. But, doing so may restrict credit extension and reduce the surplus to borrowers. Thus, the desire to reduce run-risk is balanced against the benefits from offering deposits and extending loans.

When savers are favored, the planner chooses a more equity-intensive capital structure, a more liquidity-intensive asset allocation, and a lower level of lending. Although more deposit-taking requires higher deposit rates that compress bank profit margins and discourage equity issuance, cutting lending allows the banker to raise loan rates. The higher loan rates offset the higher deposit costs and equity issuance is still possible. The deposits that are not channeled to lending are invested in the liquid assets, further reducing run-risk and offsetting some of the increase in deposit rates.

By contrast, when borrowers are favored, the planner shifts to a relatively more lending-intensive asset allocation and a higher level of lending, while choosing a more equity-intensive capital structure. Increasing lending by raising more deposits would compress profits and reduce the incentives to provide equity. So, instead, the planner reduces the liquid asset holdings and shifts funding away from deposits to release resources for loan extension and maintain the incentives for equity issuance.

Fourth, individual regulations can be used to move the private choices closer to those that a planner would choose. But no single regulation can deliver the planning outcomes. Capital and liquidity regulations reduce run-risk, but inefficiently restrict credit creation. Moreover, as banks 
take excessive risk on both sides of their balance sheet, controlling risk on one side may result in risk materializing on the other side. To replicate the planner's preferred allocations, a combination of three regulations are needed to correct the three distortions associated with the bank's asset allocation, capital structure, and level of lending that is deposit funded. The asset and liability composition distortions can be corrected using a capital and a liquidity requirement. Capital and liquidity requirements are jointly helpful and should be treated as complementary, since they operate on different sides of the balance sheet. Neither are very effective at boosting the level of lending, which the planner may favor in order to expand lending and help borrowers. To boost (or shrink) lending, other regulations, such as deposit or lending subsidies/taxes, would be needed.

Related literature. Our analysis of runs can be contrasted to several other approaches in the literature. For instance, a bank-run in our setup can occur because the information about fundamentals is very bad. Thus, our analysis can be compared with many prominent papers that analyze information-based runs such as Chari and Jagannathan (1988), Jacklin and Bhattacharya (1988), Allen and Gale (1998), and Uhlig (2010). According to this fundamental view of runs, policy interventions are needed to correct pecuniary externalities arising from missing markets; otherwise the private equilibrium is constrained efficient (Allen and Gale, 2004). Although we have abstracted from pecuniary externalities, the fundamental run probability in our model depends on the bank's liquidity and capital. Thus, regulation could be used to address not only the adverse consequences of any pecuniary externalities, but also the probability that they arise.

However, a bank-run can also occur because of a coordination problem among depositors even if the bank is solvent. This type of run can be interpreted as being panic-based, hence it is important to know what determines the panic. In the DD model, panics are a multiple equilibrium outcome. There are several ways to tackle the multiplicity of equilibria such that the model is suitable for policy analysis. Cooper and Ross (1998), Peck and Shell (2003), and Diamond and Kashyap (2016) consider that the probability of a bank-run is driven by sunspots. Ennis and Keister (2005) take an axiomatic approach to equilibrium selection and link the probability of a particular equilibrium being played to the appropriately defined incentives of agents. Despite the fact that these approaches may be, to varying degrees, adequate for certain types of positive analysis, the derivation of optimal policy requires micro-foundations for the incentives to run on the bank.

In order to address such concerns, we follow the global games approach, developed by Carlsson and van Damme (1993) and Morris and Shin (1998) and applied to bank runs by Goldstein and Pauzner (2005), to derive a unique probability of run. This approach ties run incentives to the fundamentals of the economy, the balance sheet structure of the bank, and the equilibrium payoffs to depositors withdrawing early and late. Rochet and Vives (2004) and Vives (2014) also take a global game approach but assume simpler payoffs for depositors that wait and depositors that withdraw, which are exogenous and do not depend on equilibrium allocations and interest rates. Despite the micro-foundation for run dynamics, these approaches are arguably not adequate to study optimal regulation, because deposit rates, which are important for welfare in general equilibrium, do not directly influence the incentives of depositors to run. 
Goldstein and Pauzner (2005) consider the impact of deposit contracts on run incentives but assume a fixed liquidation value for loans and an exogenously-set upper dominance region for the global game. We propose a new way to endogenize the liquidation value and the upper dominance region. Apart from the new type of global game we consider, we also differ from the aforementioned papers because we endogenize the supply of deposits, equity issuance, and loan demand. Hence, we allow the deposit and loan rates to be determined in equilibrium and respond to regulations. As we show, this is important in order to capture the welfare effects on different agents, assess the consequences of banks' private choices, and determine how a planner would alter these choices.

We should note that both in our model and the literature we have surveyed so far, deposit rates cannot be indexed by the realization of the state of the economy. Instead, if contracts could incorporate suspension clauses as in Ennis and Keister (2009) and Keister (2015), then run dynamics would differ. As noted in these papers, suspension of convertibility may not be ex post efficient, but the interaction between run dynamics, ex post intervention, and ex ante convertible deposit contracts is an interesting topic for future research.

Our analysis abstracts from policy interventions such as deposit insurance and bank resolution. Government guarantees may be useful policy interventions to reduce run-risk, but it is unclear whether they would unambiguously improve outcomes, because removing the disciplining role of deposits can encourage risk-taking (see Cooper and Ross, 2002). Allen et al. (2015) study the implications of deposit insurance in a global games framework, with a simpler banking sector than ours, while Davila and Goldstein (2022) compute the optimal deposit insurance under a sufficient statistics approach. Schilling (2022) studies the optimal bank resolution using a global games framework and finds that aggressive intervention may be counterproductive.

Finally, there is a growing literature studying the complementarity of capital and liquidity regulations, such as Walther (2016) and Kara and Ozsoy (2019) in the presence of fire-sale externalities, and Van den Heuvel (2022) in a real business cycle model with risk taking. Our conclusion, relevant to this literature, is that the number of optimal regulatory interventions is equal to the number of distorted margins rather than the number of externalities in the model. In other words, it does not make much difference if the same externality or multiple externalities distort multiple margins in distinct ways. What is important is whether different margins are distorted.

\section{Model}

The model consists of three periods, $t=\{1,2,3\}$, features a single consumption good, and includes three types of agents. In particular, there is a continuum of entrepreneurs, a continuum of savers, and one banker. We will be referring to individual entrepreneurs and savers as the entrepreneur (E) and the saver (S), and we will be also using the singular or plural for these agents depending on the context. But it should be clear that each of these agents is atomistic and representative of her type, taking equilibrium variables as given. By contrast, the banker (B) internalizes how her choices affect the equilibrium variables that matter for her profitability. 
Each entrepreneur has access to a productive, but illiquid, risky technology and chooses how much to borrow to invest in it. Funds invested at $t=1$ yield $A$ per unit of investment at $t=3$ with probability $\omega$ (which we call the "good" state of the world) and zero otherwise (the "bad" state). The project delivers no output at $t=2$ but it can be liquidated for an uncertain amount $\xi \sim U[\underline{\xi}, \bar{\xi}]$ with $0<\underline{\xi}<1<\bar{\xi}$, which is realized at $t=2$. Define $\Delta_{\xi} \equiv \bar{\xi}-\underline{\xi}$. All projects of individual entrepreneurs yield the same payoff at $t=3$ and have the same liquidation value at $t=2$. Hence, we are always referring to the representative entrepreneur and the representative project.

The banker manages an institution (bank) that stands between the entrepreneurs and savers. The bank is funded with equity from the banker and deposits from savers. The funds raised at $t=1$ are invested into either a liquid storage asset or in a loan to entrepreneurs. The loan contract with the entrepreneur specifies an uncontingent loan rate and it is backed by the entrepreneur's project and the cash flows it generates. If the entrepreneur fails to repay the loan, the bank can seize the project and any cash flows that have been generated. Loans are also callable, i.e., the bank does not need to wait for the project to mature, but can request full repayment at any point in time.

Moreover, the banker decides whether to monitor the entrepreneur's project at $t=3$ or not. Monitoring is essential because the payoff realization is private information to the entrepreneur. Without monitoring, the entrepreneur would report the bad state of the world and default. Because individual projects' payoffs are perfectly correlated, the banker needs to incur the monitoring cost once for the whole loan portfolio, which we assume is independent of how many loans are extended.

Savers are identical ex ante and each of them has a large endowment at $t=1$ that is used to fund initial consumption and savings. Each saver receives an idiosyncratic preference shock at $t=2$ to consume early or late as in DD. Given that the preference shocks are independent and identically distributed, some fraction of savers will need to consume at $t=2$ and the rest wait to consume at $t=3$. Thus, savers are ex ante identical, but ex post heterogeneous, which we will refer to as impatient and patient, respectively. The (ex ante) representative saver invests in bank deposits or holds a liquid storage asset at $t=1$. Deposits are demandable, which is important to provide incentives to the banker to monitor as we shall explain later.

A loan can be recalled to pay deposits. Upon being recalled it yields an immediate gross amount $\xi$. When a loan is called, the entrepreneur forfeits the portion of the project that is funded by the loan. Depending on the value of $\xi$, the bank may not have enough resources to fully pay any level of withdrawals, while monitoring incentives may be weak or strong. Aside from extremely high or low realizations of $\xi$, the bank is at risk for self-fulfilling runs: A patient saver will demand her deposits early if she believes that other patient savers will do the same. To address the coordination problem and obtain a unique equilibrium, we assume that savers receive noisy signals about the true realization of $\xi$ at $t=2$. These signals not only provide information about the fundamental $\xi$, but also about the beliefs of other savers, and so serve to coordinate the patient savers' decisions. We show that there is a threshold strategy that patient savers will follow such that when the signal is above a threshold value they do not withdraw early and when it is below the threshold they run.

Moreover, based on the signal the savers form posterior beliefs about $\xi$ and we show that there 
is a unique threshold $\xi^{*}$, such that all patient savers withdraw their deposits when they believe the true realization is below that value and they keep their deposits in the bank otherwise. We will refer to $\xi^{*}$ as the run threshold and denote the probability of a run by $q=\left(\xi^{*}-\underline{\xi}\right) / \Delta_{\xi}$.

The liquidation value may be understood in several ways. For instance, the incomplete project could have a secondary use in the interim period because it can be used in conjunction with an alternative short-term technology. Alternatively, we could assume that it can be sold to some outside investors as in Shleifer and Vishny (1992). In other words, $\xi$ does not strictly represent the salvage value, but rather the liquidation/resale value of long-term investment. The upper bound $\bar{\xi}$ has to be high enough that the bank can always withstand a panic for some realizations. While the lower bound $\underline{\xi}$ has to be low enough that the bank may run out of liquidity even if a panic does not occur. We describe the importance of these bounds in section 2.4.

We simplify the model in three ways by assuming that savers have quasi-linear preferences, entrepreneurs have linear preferences and no initial endowment, and the production technology is linear. The main cost of linearity is that it may leave agents at a point where they break even from their savings and borrowing choices. Hence, the bank creates no value for them, which does not distort the basic properties of the private equilibrium, but has powerful implications for regulation. We reintroduce curvature into the savers' and entrepreneurs' problems in other ways that make the results less extreme while keeping the model tractable. Hereafter, we explain the implications of each of these assumptions, but none of them are crucial for our main results.

Figure 1 presents the timeline of the model. Sections 2.1-2.4 describe the agents' optimization problems and the derivation of the run threshold. Section 2.5 characterizes the private equilibrium.

\begin{tabular}{|c|c|c|}
\hline$t=1$ & $t=2$ & $t=3$ \\
\hline $\mathrm{E}$ borrow and invest in risky projects & S learn their type & Investment payoff is determined \\
\hline $\mathrm{S}$ invest in demandable bank deposits & $S$ receive noisy signals about $\xi$ & E privately learn the payoff realization \\
\hline $\begin{array}{l}\text { and potentrally in the liquid asset } \\
\text { B raises equity and deposits and } \\
\text { invests in loans and liquid assets }\end{array}$ & $\begin{array}{l}\text { and decide whether to withdraw } \\
\text { B recalls loans and pays withdrawals } \\
\text { If } \xi<\xi^{*} \text {, a run occurs }\end{array}$ & $\begin{array}{l}\text { B decides whether to monitor } \\
\text { Loans and deposits are repaid if the } \\
\text { payoff is } A \text { and default otherwise }\end{array}$ \\
\hline
\end{tabular}

Figure 1: Timeline

\subsection{Savers}

Savers are endowed with $e_{S}$ at $t=1$ and decide how much to invest in bank deposits, $D$, and how much to hold in the liquid asset, $L_{S}$. At $t=2$, a portion of savers, $\delta$, receive a preference shock to consume immediately, while the rest, $1-\delta$, want to consume at $t=3$. These shocks are private information, are independent and identically distributed, and are not contractible ex ante.

Deposits are demandable, early withdrawals are serviced sequentially, and the $t=2$ and $t=$ 3 uncontingent gross interest rates are $r_{D}$ and $R_{D}$, respectively. We restrict all gross rates to be higher than one, an assumption that we relax in our extensions. This contract structure creates the 
possibility of a run, since patient savers may choose to demand their deposits early depending on their own information and their expectations about the actions of other patient savers.

If there is no run, i.e., $\xi \in\left[\xi^{*}, \bar{\xi}\right]$, only impatient depositors withdraw, and they receive $D r_{D}$. Patient depositors' repayments will depend on the state realization at $t=3$; they receive their promised payment, $D R_{D}$, with probability $\omega$ and zero otherwise, since $B$ defaults. In a run, all depositors attempt to withdraw, and there is probability $\theta(\xi)$, defined later, that any depositor gets $D r_{D}$.

Savers have quasi-linear preferences for consumption, such that they value consumption linearly at $t=2$ and $t=3$. This is our first assumption that greatly simplifies the patient savers' decision to join a run, because it means that all that must be computed is the expected payoff from deposits at $t=$ 2 versus $t=3$. By contrast, if savers were risk averse, finding the threshold that determines whether to run is much more complicated. The complication arises because a saver needs to compute her expected utility differential between waiting and withdrawing, accounting for all possible out-ofequilibrium beliefs about the actions of other savers. Computing the expected deposit payoffs is simpler than computing the expectation of a nonlinear function of the deposit payoffs. Moreover, quasi-linearity yields a very tractable deposit supply schedule, which substantially simplifies the normative analysis. The disadvantage of linearity is that it reduces the usefulness of a deposit to a saver. Essentially, the deposit becomes a pure financial instrument whose only value is that it pays more interest than the liquid asset. Put differently, the deposit would be equivalent to a bond.

To make deposits more useful, we assume that impatient savers enjoy transaction benefits from having deposits when there is no run. To do this, we suppose that this advantage is described by a concave function $\mathrm{V}$ that is increasing in the amount promised and repaid, $D r_{D}$, and it only accrues for $r_{D} \geq 1$, providing some justification for the assumption on gross returns. Peck and Shell (2010) also have a similar assumption about the utility from the transaction services of deposits. This additional benefit of deposits partially offsets the stark implications of quasi-linear preferences. Modeling things this way leads to no qualitative changes relative to a model with concave utility and no transactions services but makes it substantially easier and faster to solve the model.

The expected utility of an individual saver is given by

$$
\begin{aligned}
\mathbb{U}_{S} & =\overbrace{U\left(e_{S}-D-L_{S}\right)}^{\text {Utility at } t=1}+\overbrace{\int_{\underline{\xi}}^{\xi^{*}}\left[\beta \delta+\beta^{2}(1-\delta)\right]\left[\theta(\xi) D r_{D}+L_{S}\right] \frac{d \xi}{\Delta_{\xi}}}^{\text {run }} \\
& +\underbrace{\int_{\xi^{*}}^{\bar{\xi}} \beta \delta\left[D r_{D}+L_{S}\right] \frac{d \xi}{\Delta_{\xi}}}_{\text {no run,impatient }}+\underbrace{\int_{\xi^{*}}^{\bar{\xi}} \beta^{2}(1-\delta)\left[\omega D R_{D}+L_{S}\right] \frac{d \xi}{\Delta_{\xi}}}_{\text {no run,patient }}+\underbrace{\int_{\xi^{*}}^{\int_{\xi}} V\left(D r_{D}\right) \frac{d \xi}{\Delta_{\xi}}}_{\text {transaction services }} .
\end{aligned}
$$

$U$ is the utility function for $t=0$ consumption with $U^{\prime}>0$ and $U^{\prime \prime}<0$; $V$ captures the transaction services of deposits with $V(0)=0, V^{\prime}>0$ and $V^{\prime \prime}<0 ; \beta \leq 1$ is the time-discount factor. ${ }^{2}$

Savers choose the level of deposits and their holdings of the liquid asset to maximize (1). An individual saver takes the run threshold, $\xi^{*}$, and the probability of being repaid in a run, $\theta(\xi)$, as

\footnotetext{
${ }^{2}$ The transaction services only accrue to the impatient depositors, so $\delta$ is subsumed inside $V$.
} 
given. These objects depend on the aggregate bank portfolio, and we suppose that the individual saver is atomistic so as to not account for her impact on them. In contrast, a social planner would internalize the effect of the choices. Nevertheless, individual savers have rational expectations and correctly anticipate the equilibrium level of run-risk when making their decisions. Finally, shortselling of deposits and the liquid asset is not allowed, i.e., $D \geq 0$ and $L_{S} \geq 0$.

The optimal choice of deposits by $S$ yields the following deposit supply (DS) schedule:

$$
\begin{aligned}
U^{\prime}\left(e_{S}-D-L_{S}\right) & \geq\left[\beta \delta+\beta^{2}(1-\delta)\right] r_{D} \int_{\underline{\xi}}^{\xi^{*}} \theta(\xi) \frac{d \xi}{\Delta_{\xi}} \\
& +\left[\beta \delta r_{D}+\beta^{2}(1-\delta) \omega R_{D}+V^{\prime}\left(D r_{D}\right) r_{D}\right](1-q),
\end{aligned}
$$

which holds with strict equality if savers choose to hold deposits in equilibrium, i.e., $D>0$. Condition (2) says that savers equate the marginal utility of forgone consumption at $t=1$ to the expected marginal utility gain from holding deposits in the future. In a run, all savers withdraw; an individual saver will receive $r_{D}$ per unit of deposits with probability $\theta(\xi)$ for each realization of $\xi<\xi^{*}$. Otherwise, with probability $1-q$, a run does not occur, and only impatient savers withdraw. In this case, an individual saver is either impatient with probability $\delta$ and receives the period 2 deposit rate $r_{D}$ or is patient with probability $1-\delta$ and receives the period 3 deposit rate $R_{D}$ with probability $\omega$. Absent a run, $S$ also enjoys the marginal benefit of transaction services, $V^{\prime}\left(D r_{D}\right) r_{D}$.

Savers may also self-insure by holding the liquid asset. The optimal $L_{S}$ is given by:

$$
U^{\prime}\left(e_{S}-D-L_{S}\right) \geq \beta \delta+\beta^{2}(1-\delta) .
$$

This condition compares the marginal loss in consumption to the expected value of storage and holds with strict equality if $L_{S}>0$. Without loss of generality, we consider equilibria in which $L_{s}=0$ but $D>0$, i.e., (2) holds with equality, while (3) is slack. Savers will not use the storage technology whenever (i) their endowments are not excessive, (ii) the bank offers high enough deposit rates, or (iii) transaction services are sufficiently valuable.

Substituting the deposit schedule (2) into (1), we get the indirect utility function,

$$
\mathbb{U}_{S}^{*}=U\left(e_{s}-D\right)+U^{\prime}\left(e_{s}-D\right) D+(1-q)\left[V\left(D r_{D}\right)-V^{\prime}\left(D r_{D}\right) D r_{D}\right]
$$

for the benchmark case in which $L_{S}=0 .{ }^{3}$ Given our assumptions about $V()$, it is easy to show that the third term in (4) is strictly positive. ${ }^{4}$ Moreover, the first two terms in (4) must be higher than $\mathbb{U}_{S}^{\alpha}$ (the utility level from saving only using liquid assets), otherwise savers could choose $L_{S}>0$ and attain that level of utility. Hence, savers are always better off using the bank compared to autarky.

\footnotetext{
${ }^{3}$ For completeness, if $L_{S}>0$ then the savers' indirect utility is $\mathbb{U}_{S}^{\alpha}+(1-q)\left[V\left(D r_{D}\right)-V^{\prime}\left(D r_{D}\right) D r_{D}\right]$, where $\mathbb{U}_{S}^{\alpha}$ is the utility in autarky given by $\mathbb{U}_{S}^{\alpha}=U\left(U^{\prime-1}\left(\beta \delta+\beta^{2}(1-\delta)\right)\right)+\left[\beta \delta+\beta^{2}(1-\delta)\right]\left[e_{S}-U^{\prime-1}\left(\beta \delta+\beta^{2}(1-\delta)\right)\right]$.

${ }^{4}$ Since $D>0$, the term can be written as $(1-q) D\left[V(D) / D-V^{\prime}(D)\right]$. Because $V(0)=0, V(D) / D$ is the slope of the straight line emanating from zero and passing through $D$. Since $V$ is strictly concave, its image is always above the image of the straight line for any point $x \in(0, D)$. Because $V^{\prime}$ is strictly decreasing, $V$ will necessarily cross the straight line connecting zero and $D$ from above. Hence, the derivative of $V$ at $D$ is strictly smaller than the slope of the straight line.
} 
Even with our simplifying assumptions, this framework produces an equilibrium and a set of decision rules for savers that possess, intuitive properties. In particular, savers use the bank because it offers a better way to save for the future and facilitates transactions. The former is captured by the benefit in terms of period 1 consumption (the first two terms in (4)). The latter is captured by the benefit from the transaction services (the last term in (4)).

\subsection{Entrepreneurs}

Entrepreneurs have the rights to operate real projects that are in perfectly elastic supply, require a unit of funding at $t=1$, are infinitely divisible when liquidated, and mature at $t=3$. For simplicity, $E$ does not have an endowment but borrows $I$ from the bank at gross rate $R_{I}$ to invest in the risky technology. Moreover, $E$ is risk neutral and derives utility only from consumption at $t=3$. Finally, $E$ is protected by limited liability when projects mature and loans are due.

The risk neutrality of entrepreneurs together with the absence of initial endowment is our second technical assumption. Risk neutrality means that the entrepreneur cares only about the expected profits from operating the technology. The absence of an endowment means that the decision to default will depend only on the productivity of the technology, not on entrepreneurs' leverage. Otherwise, the savers' run decision becomes more intertwined with how much the entrepreneur wants to borrow and the relative risk-aversion of the two parties will matter for how risks are shared.

The linearity of the production function is our third technical assumption, which simplifies the derivation of the loan demand. Yet, the linearity also means that $R_{I}$ depends only on $A$. If the production function had curvature, it would substantially complicate the calculations. The complication arises because the realized production depends on the bank's decision to recall loans, which occurs after the realization of $\xi$ is known. The loan demand trades off the expected revenue from borrowing to produce against the cost of repaying the loan. And it is easier to compute the expected profits to entrepreneurs over $\xi \geq \xi^{*}$ when production is linear rather than when it is a nonlinear function of the number of projects that are not recalled.

In order to make loan demand less mechanical, while maintaining analytical tractability, we introduce a cost of effort that the entrepreneur incurs upon investing. We suppose that this cost is incurred before the payoff from the investment is known; we could also call this cost an adjustment cost. We assume that this cost is convex and it pertains to total investment.

The expected utility of an individual entrepreneur can be written as:

$$
\mathbb{U}_{E}=\int_{\xi^{*}}^{\bar{\xi}}\{\omega[\underbrace{A(1-y(\xi, \delta)) I}_{\text {realized output }}-\underbrace{(1-y(\xi, \delta)) I R_{I}}_{\text {loan obligation }}]-\underbrace{c(I)}_{\text {effort }}\} \frac{d \xi}{\Delta_{\xi}},
$$

where $y(\xi, \delta)$ is the portion of loans recalled to serve withdrawals from impatient savers (and will be derived below). If a run does not occur, $E$ repays the outstanding loans in the good state, (1 $y(\xi, \delta)) I$, as long as the per unit payoff, $A$, is higher than the promised gross loan rate $R_{I}$. Naturally, $E$ defaults in the bad state when the project pays zero. In a run, all projects funded by bank loans are 
liquidated and no production takes place. Finally, $c(I)$ is the effort/adjustment cost with $c(0)=0$, $c^{\prime}>0$ and $c^{\prime \prime}>0$. E needs to incur this cost in order to produce before she learns the realization of the state of the world and after run uncertainty has been resolved. Hence, $E$ will choose not to exert effort and thereby avoid the cost in a run. Absent a run, given that the net payoff to $E$ is increasing in $\xi$, she will choose to produce if the following incentive compatibility constraint holds:

$$
\omega\left[A-R_{I}\right]\left(1-y\left(\xi^{*}, \delta\right)\right) I-c(I)>0 .
$$

Entrepreneurs choose the level of investment and, thus, borrow, $I$, to maximize (5). An individual entrepreneur takes the run threshold, $\xi^{*}$, and the aggregate portion of loans recalled, $y(\xi, \delta)$, as given. These objects depend on the aggregate bank portfolio, and we suppose that individual entrepreneurs are atomistic so that they do not account for their impact on them. A social planner would internalize the effect of the choices. However, individual entrepreneurs have rational expectations and correctly anticipate the equilibrium level of run-risk when making their decisions.

The optimal choice of $I$ by $E$ yields the following loan demand (LD) schedule:

$$
\int_{\xi^{*}}^{\bar{\xi}}\left\{\omega\left[A-R_{I}\right](1-y(\xi, \delta))-c^{\prime}(I)\right\} d \xi / \Delta_{\xi}=0 .
$$

Condition (7) says that $E$ equates the expected profit margin on the remaining projects-given by the difference between the marginal product of investment and the gross loan rate-to the marginal effort cost over the realizations of $\xi$, where a run does not materialize.

Substituting the loan demand schedule (7) in (5), we get the following indirect utility function

$$
\mathbb{U}_{E}^{*}=(1-q)\left[c^{\prime}(I) I-c(I)\right]
$$

Given our assumptions about $c$, it is easy to show that (8) is always strictly positive, i.e., $E$ is strictly better off than in autarky, where $E^{\prime} s$ utility is zero. ${ }^{5}$ If effort is costless, then $\mathbb{U}_{E}^{*}=0$, and the loan rate is equated to the marginal product of the project, i.e., $R_{I}=A$ from (7).

Despite our simplifying assumptions about entrepreneurs' preferences and shape of the production function, entrepreneurs' choices are very conventional. Their welfare in equilibrium, given by (8), is increasing in $I$ and decreasing in $q$. Our setup captures these relationships in a tractable way and succinctly identifies the distortions that any policies will aim to correct.

\subsection{Banker and Bank}

The banker $(B)$ makes all investment and funding decisions to maximize her own utility. At $t=1$, she is endowed with $e_{B}$ and decides how much equity, $E$, to put into the bank. In addition, she

\footnotetext{
${ }^{5}$ To show that (8) is strictly positive, it suffices to show that $c^{\prime}(I)-c(I) / I>0$ given that $I>0$; otherwise, $E$ is in autarky (short-selling of projects is not allowed). Because $c(0)=0, c(I) / I$ is the slope of the straight line starting at zero and passing through $I$. Given that $c$ is strictly convex, its image is always below the image of the straight line for any point $x \in(0, I)$. Because $c^{\prime}$ is strictly increasing, $c$ will necessarily cross the straight line connecting zero and $I$ from below and, hence, the derivative of $c$ at $I$ is strictly higher than the slope of the straight line.
} 
decides how many deposits to raise, $\mathrm{D}$, and how much to invest in the liquid assets, $L$, and illiquid loans, $I$, subject to the following balance sheet $(B S)$ constraint:

$$
I+L=D+E
$$

If a bank-run occurs at $t=2$, i.e., $\xi<\xi^{*}$, the bank is liquidated and the proceeds are distributed on a first-come, first- served basis. The maximum level of withdrawals that can be served in full is

$$
\theta(\xi)=\frac{\xi I+L}{D r_{D}}
$$

which is also equal to the probability of being served if all depositors withdraw.

If there is not a run, i.e., $\xi \geq \xi^{*}$, the balance sheet and profits after $t=2$ depend on the realization of $\xi$ and the level of withdrawals, $\lambda$. The bank will recall and liquidate a portion $y(\xi, \lambda)$ of its loan portfolio to serve the early withdrawals. ${ }^{6}$ The amount recalled is given by

$$
y(\xi, \lambda)=\max \left[\frac{\lambda D r_{D}-L}{\xi_{I}}, 0\right] .
$$

The profits that $B$ receives in the good state at $t=3$ are equal to the repayment on the remaining loans minus the payment on the remaining deposits. In the bad state, $B$ defaults, so profits are zero. $B$ needs to incur a monitoring cost, $X$, to learn the true state of the world. Alternatively, $B$ can forgo the monitoring, in which case the entrepreneur will report that productivity was zero and default, independent of state realization. Hence, there is a moral hazard problem, akin to Holmström and Tirole (1997), such that $B$ will choose to monitor only if expected profits exceed $X$.

Given a level for the liquidation value $\xi$ and the level of withdrawals $\lambda, B$ will choose to monitor if the following incentive compatibility constraint is satisfied:

$$
\omega\left[(1-y(\xi, \lambda)) I R_{I}-(1-\lambda) D R_{D}\right]-X \geq 0 .
$$

The monitoring decision is taken before $B$ learns the true state of the world, so incentive compatibility requires that the expected payoff to $B$ is higher than $X$. In an equilibrium where the bank is taking deposits to make loans, (12) must be satisfied when a run does not occur, i.e., for $\xi \geq \xi^{*}$ (and $\lambda=\delta$ ); otherwise monitoring would not take place, depositors would always run, and there is no gain to operating the bank. In Section 2.4, we prove that (12) is always slack for $\xi \geq \xi^{*}$.

The banker's expected utility is given by:

$$
\mathbb{U}_{B}=W\left(e_{B}-E\right)+\int_{\xi^{*}}^{\bar{\xi}}\{\omega[\underbrace{(1-y(\xi, \delta)) I}_{\begin{array}{c}
\text { outstanding } \\
\text { loans }
\end{array}} \underbrace{R_{I}}_{\begin{array}{c}
\text { loan } \\
\text { rate }
\end{array}}-\underbrace{(1-\delta) D}_{\begin{array}{c}
\text { patient } \\
\text { deposits }
\end{array}} \underbrace{R_{D}}_{\begin{array}{c}
\text { deposit } \\
\text { rate }
\end{array}}]-\underbrace{X}_{\begin{array}{c}
\text { monit. } \\
\text { cost }
\end{array}}\} \frac{d \xi}{\Delta_{\xi}},
$$

\footnotetext{
${ }^{6}$ The bank might also want to liquidate loans beyond the need to serve early withdrawals and carry the proceeds forward using the storage technology. But this would only occur if the realization of $\xi$ is higher than the expected return from holding the loan to maturity, which we rule out by assumption.
} 
where $W()$ is $B^{\prime} s$ utility function for $t=1$, with $W^{\prime}()>0$ and $W^{\prime \prime}()<0$. The banker has also quasi-linear preferences, but, unlike the saver, never needs to consume in the interim period.

\subsection{Global Game and Bank-Run Threshold}

Having described the choices of all the agents, we can now determine when individual patient savers will want to withdraw their deposits at $t=2$. The source of fundamental uncertainty in our model is the liquidation value $\xi$ for which individual patient savers receive a private noisy signal. The decision depends not only on each saver's belief about the bank's financial health, but also on their beliefs about how other savers will behave given their individual signals. We show that a unique threshold signal exists such the individual savers withdraw only if their own signal is lower than that threshold. As the noise in private signals goes to zero, this threshold converges to a unique threshold for fundamentals $\xi^{*}$, which we have used in the analysis so far.

We prove this result in six steps using a series of Lemmas and Propositions. First, we derive the regions of $\xi$ where savers act unilaterally, i.e., without considering others actions. Lemmas 1 and 2 establish these regions, known as the lower and upper dominance regions. In the former, an individual patient saver decides to withdraw even if no other patient savers withdraw, while in the latter she does not withdraw even if all savers withdraw. Second, we show that for intermediate values of $\xi$ individual actions depend on beliefs about aggregate withdrawals, as they govern the bank's incentive to monitor and whether the bank has adequate liquidity to meet withdrawals (Lemma 3). Third, we show that if a threshold strategy is followed by all savers, then individuals can form beliefs about aggregate withdrawals and compute unique cutoffs for the regions of $\xi$ where the bank is expected to monitor and have enough liquidity to meet withdrawal requests (Lemma 4). Equipped with these beliefs and cutoffs, individuals can compute the expected payoff from withdrawing given their private signals and posteriors about $\xi$. Fourth, we show that following a strategy that supposes a common threshold signal is an equilibrium. Fifth, we establish the existence and uniqueness of the threshold signal, and present the logic and mathematical characterization for our novel proof, leading to Proposition 1 that establishes the uniqueness of $\xi^{*}$. Finally, Proposition 2 summarizes the results about the existence and uniqueness of a threshold-strategy equilibrium.

Step 1. A bank is immune to runs if it has both enough liquidity to serve all potential withdrawals and maintains incentives to monitor irrespective of the level of withdrawals. The maximum level of withdrawals that the bank can sustain for given $\xi$ is equal to $\theta(\xi)$ in (10), which is increasing in $\xi$. Thus, there is a cutoff $\hat{\xi}$ such that the bank does not have enough liquidity to even serve early withdrawals for $\xi<\hat{\xi}$, and another value $\hat{\xi}$ such that it has enough liquidity to serve all potential withdrawals for $\xi \geq \hat{\hat{\xi}}$. These cutoffs are the solutions to $\theta(\hat{\xi})=\delta$ and $\theta(\hat{\xi})=1$, which yields

$$
\hat{\xi}=\frac{\delta D r_{D}-L}{I} \quad \text { and } \quad \hat{\xi}=\frac{D r_{D}-L}{I} .
$$

Turning to monitoring incentives, note that (12) is increasing in $\xi$ and for now suppose that it is decreasing in $\lambda$; we establish this below. Then, the banker will not monitor for $\xi<\xi_{l d}$ even if only 
impatient savers withdraw, while she will monitor for $\xi \geq \xi_{\text {ud }}$ even if all savers withdraw. These cutoffs are obtained by setting (12) to zero and solving for $\xi$ when $\lambda=\delta$ and $\lambda=1$, which yields

$$
\xi_{l d}=\frac{\delta D r_{D}-L}{I-(1-\delta) D R_{D} / R_{I}-X /\left(\omega R_{I}\right)} \quad \text { and } \quad \xi_{u d}=\frac{D r_{D}-L}{I-X /\left(\omega R_{I}\right)}
$$

Note that $\xi_{l d}>\hat{\xi}$ and $\xi_{u d} \geq \hat{\xi}$, with strict inequality for $X>0$. This implies that the cutoffs related to monitoring $\xi_{l d}$ and $\xi_{u d}$, rather than $\hat{\xi}$ and $\hat{\xi}$, are the candidate ones to define the lower and upper dominance regions. Consider for example a $\xi \in\left(\hat{\xi}, \xi_{l d}\right)$ and $\lambda=\delta$. The banker would not monitor even though the bank has enough liquidity to serve withdrawals, because expected profits are not high enough to cover the monitoring cost. Similarly, for $\xi \in\left(\hat{\xi}, \xi_{u d}\right)$ and $\lambda=1$, the banker would not monitor even though the bank has enough liquidity to serve withdrawals from all savers, because the bank has to liquidate so many loans that the expected profits from the remaining ones are not enough to cover the monitoring cost. To establish the lower and upper dominance regions we need to show that $\xi_{l d}, \xi_{u d} \in(\underline{\xi}, \bar{\xi})$ and that (12) is indeed decreasing in $\lambda$. The next two Lemmas provide the conditions for these properties to hold.

Lemma 1. Existence of the lower dominance region. For sufficiently high $\bar{\xi}$ and $A$, the bank has incentives to choose $\delta D>L$.

Proof. Define $z \equiv \delta D-L \in[0, \delta D]$. Using (9), we obtain $I(z)=D+E-L=z+(1-\delta) D+E$, i.e., $z$ are the loans not funded by long-term deposits and equity. We can also solve for $R_{D}$ and $R_{I}$ as functions of $z$, denoted by $R_{D}(z)$ and $R_{I}(z)$, by substituting $I(z)$ into (2) and (7). Substituting all expressions in (13) and taking the first-order condition with respect to $z$ yields

$$
\begin{gathered}
\omega R_{I}(z) \int_{\xi^{*}}^{\bar{\xi}}(1-1 / \xi) d \xi+\omega R_{I}^{\prime}(z) \int_{\xi^{*}}^{\bar{\xi}}(1-y(\xi, \delta)) I(z) d \xi-\omega R_{D}^{\prime}(z) \int_{\xi^{*}}^{\bar{\xi}}(1-\delta) D d \xi \\
+\frac{d \xi^{*}}{d z}\left(-\Pi\left(\xi^{*}\right)+\omega \frac{d R_{I}}{d \xi^{*}} \int_{\xi^{*}}^{\bar{\xi}}(1-y(\xi, \delta)) I(z) d \xi-\omega \frac{d R_{D}}{d \xi^{*}} \int_{\xi^{*}}^{\bar{\xi}}(1-\delta) D d \xi\right)+\psi_{z} \Delta_{\xi}=0,
\end{gathered}
$$

where $\psi_{z}$ is the Lagrange multiplier on $z \geq 0$ and $\Pi\left(\xi^{*}\right)=\omega\left(1-y\left(\xi^{*}, \delta\right)\right) I(z) R_{I}(z)-(1-\delta) D R_{D}(z)-$ $X$ are bank's expected profits for $\xi=\xi^{*}$. The Kuhn-Tucker condition requires that $\psi_{z} \geq 0$ for $z=0$ to be a solution, i.e., the sum of the first four terms must be negative or zero. We prove by contradiction that $z=0$ cannot be an equilibrium. To do this we sign each of the four individual terms. We then explain how by invoking the conditions in the Lemma, we can make their sum positive at the prevailing equilibrium allocations, ruling out the $z=0$ equilibrium. Finally, we guess that there is a perturbation so that there is an equilibrium with $z>0$ and verify that in our subsequent analysis.

The first term in (16) captures the direct payoff from increasing $z$ and is positive if $\int_{\xi^{*}}^{\bar{\xi}}(1-$ $1 / \xi) d \xi>0$, or $\bar{\xi}-\log \bar{\xi}>\xi^{*}-\log \xi^{*}$. Since $\bar{\xi}>1$, the left-hand side is increasing in the level of $\bar{\xi}$ and, thus given a $\xi^{*}$, the first term can be made positive for sufficiently high $\bar{\xi}$. The second and third term capture the direct effect of higher $z$ on $R_{I}$ and $R_{D}$ and are both negative. From (7), we can obtain $R_{I}^{\prime}(z)=-\left[\alpha_{1} c^{\prime}(z+(1-\delta) D+E)+\alpha_{2} c^{\prime \prime}(z+(1-\delta) D+E)\right]<0$, because $\alpha_{1}$ and $\alpha_{2}$ are 
positive. ${ }^{7}$ From (2), we get $R_{D}^{\prime}(z)=\left(\beta \delta+\beta^{2}(1-\delta) /\left((1-q) \omega \beta^{2}(1-\delta)\right) \int_{\xi}^{\xi^{*}}(1-\xi) / D d \xi / \Delta_{\xi}>0\right.$.

The fourth term captures the effect of higher $z$ on the run threshold $\xi^{*}$ and operates through the three sub-terms in parenthesis multiplying $d \xi^{*} / d z$ : For a marginal increase in $\xi^{*}$, (i) the bank forgoes profits $\Pi\left(\xi^{*}\right)$ (first sub-term), (ii) $d R_{I} / d \xi^{*}<0$ from (7), meaning loan rates fall which reduces profitability (second sub-term), and (iii) $d R_{D} / d \xi^{*}>0$ from (2), implying deposit rates rise which also reduces profitability (third sub-term). In any (reasonable) equilibrium where $z$ is higher, run-risk is higher, meaning that $d \xi^{*} / d z>0$, so the whole fourth term is negative.

To find an equilibrium with $z>0$, begin with the $z=0$ solution to (16) and start increasing $A$, allowing $R_{I}$ to adjust, while keeping all other variables at the prevailing equilibrium values. Then, the first term in (16) can be increased enough so that the sum of all four terms is strictly positive. We now guess we can perturb the $z=0$ allocations by making the second, third, and fourth terms more negative and the first one less positive so that (16) holds with equality for $\psi_{z}=0$ and $z>0$. While performing this calculation, we kept other variables at their $z=0$ values. Thus, we need to verify that when all the other variables are optimally chosen and $z>0$, the Lemma is still true. For now we guess that this is the case and verify it in Section 3.2.

The intuition underlying the Lemma is simple. $\delta D-L$ are the loans that are not funded by longterm deposits and equity, so that they represent loans that are liquidated to serve early withdrawals. If lending is profitable enough and loans can be potentially liquidated at high enough values, then planning to liquidate some loans to meet early withdrawals is desirable. Given that $r_{D} \geq 1$, the Lemma implies that $\xi_{l d}>0$ and, thus, establishes the existence of a lower dominance region because we can choose a $\underline{\xi}$ sufficiently close to zero such that $\underline{\xi}<\xi_{l d}{ }^{8}$

Lemma 2. Existence of the upper dominance region. For sufficiently low $V^{\prime}$ and high $e_{S}$, there exists $\xi_{\text {ud }}<R_{I} r_{D} / R_{D}$ and (12) is decreasing in $\lambda$.

Proof. Using the definition of $\xi_{\text {ud }}$ from (15), the claim we want to establish, becomes

$$
I R_{I} r_{D}-D R_{D} r_{D}+L R_{D}-X / \omega r_{D}>0 .
$$

For $r_{D}=1$, using the balance sheet constraint (9) and Lemma 1, (17) becomes $(D-L)\left(R_{I}-R_{D}\right)+$ $E R_{I}-X / \omega>(1-\delta) D\left(R_{I}-R_{D}\right)+E R_{I}-X / \omega>0$, provided $R_{I}-R_{D}>0$. This condition is intuitive: It says that $B$ 's profits from offering late deposits plus investing her own equity are positive after monitoring costs; otherwise, the bank would not operate. If $I R_{I}-D R_{D}-X / \omega>0$, (17) is also increasing in $r_{D}$ so no further assumptions are needed. If this is not true, (17) would, by continuity,

$$
{ }^{7} \alpha_{1}=\left(\omega \int_{\xi^{*}}^{\bar{\xi}}\left(1-y\left(\xi^{*}, \delta\right)\right) d \xi\right)^{-1}<0 \text { and } \alpha_{2}=-\omega \int_{\xi^{*}}^{\bar{\xi}} \frac{\xi\left(E+(1-\delta) D+\delta D\left(r_{D}-1\right)\right.}{(\xi(z+(1-\delta) D+E))^{2}} d \xi\left(\omega \int_{\xi^{*}}^{\bar{\xi}}\left(1-y\left(\xi^{*}, \delta\right)\right) d \xi\right)^{-2}<0 .
$$

${ }^{8}$ The liquidity regulations that have been implemented since the global financial crisis, such as the Liquidity Coverage Ratio (LCR), still allow for banks to choose $\delta D>L$, so that Lemma 1 can continue to hold. This is because these regulations pertain to banks' expected net liquidity needs. In particular, regulatory liquidity is calculated by summing the liquid assets that banks hold ( $L$ in our model) plus some less liquid assets (such corporate bonds or payments coming due from all other banking activities) and subtracting expected fund outflows ( $\delta D$ in our model). If the realized level of inflows is unexpectedly small, then $L$ will be far below the level of expected deposit outflows. 
hold for $r_{D}>1$ for $r_{D}$ sufficiently close to one. The latter will hold if the marginal benefits from payments services, captured by $V^{\prime}$ in the $D S$ schedule (2), are sufficiently small with $e_{S}$ sufficiently high. We verify that in the equilibria we study that these conditions hold.

Lemma 2 implies that there is $\bar{\xi}$ higher than $\xi_{u d}$, such that the upper dominance cutoff exists, but with $\bar{\xi}$ less than $R_{I} r_{D} / R_{D}$. The upper bound on $\bar{\xi}$ ensures that liquidating a loan and storing the proceeds is less attractive than letting the loan mature. Hence, the bank's profits given by (12), are decreasing in $\lambda$ for all $\xi$ (because the derivative of (12) with respect to $\lambda$ is $-R_{I} r_{D} / \xi+R_{D}<0$ ).

Taken together, Lemmas 1 and 2 guarantee that $\xi_{l d}$ and $\xi_{u d}$ are indeed the appropriate lower and upper dominance cutoffs. Figure 2 shows graphically the lower and upper dominance regions.

\begin{tabular}{l|l|l}
$\xi=\underline{\xi}$ & $\xi=\xi_{l d}$ & \multicolumn{2}{c}{$\xi=\xi_{\text {ud }}$} \\
\hline Banker never monitors & Monitoring depends on level of withdrawals & Banker always monitors \\
Lower dominance region & Strategic complementarities \& multiple equilibria & Upper dominance region
\end{tabular}

Figure 2: Lower and upper dominance regions

Step 2. For $\xi \in\left[\xi_{l d}, \xi_{u d}\right)$, the incentives to monitor depend on the level of withdrawals, and a patient saver may decide to withdraw early if she believes that other patient savers will withdraw as well. The strategic complementarity in the withdrawal decision creates multiple equilibria. The withdrawal decision depends on the perceived payoff differential between waiting until $t=3$ and withdrawing at $t=2$. We denote this differential by $v(\xi, \lambda)$ and report in (18) the value it takes for different beliefs about the level of withdrawals $\lambda$ when the realization of fundamentals is equal to $\xi$ :

$$
v(\xi, \lambda)=\left\{\begin{array}{lll}
\omega D R_{D}-D r_{D} & \text { if } & \hat{\lambda}(\xi) \geq \lambda \geq \delta \\
-D r_{D} & \text { if } & \min (\theta(\xi), 1) \geq \lambda>\hat{\lambda}(\xi) \\
-(\xi I+L) / \lambda & \text { if } & 1 \geq \lambda>\min (\theta(\xi), 1)
\end{array}\right.
$$

We discuss the three regions in (18) in turn. The first case concerns beliefs for $\lambda$ under which the banker has an incentive to monitor, so that full repayment at $t=3$ is possible. The maximum level for which this is true is given by

$$
\hat{\lambda}(\xi)=\frac{(\xi \cdot I+L) R_{I}-\xi\left(D R_{D}+X / \omega\right)}{D\left(R_{I} r_{D}-\xi R_{D}\right)} .
$$

with $d \hat{\lambda}(\xi) / d \xi>0$ using the chain rule and Lemma 2. The payoff differential in this region is $\omega D R_{D}-D r_{D}$, which is strictly positive, otherwise no patient savers would ever wish to wait. The remaining regions have no monitoring. The second one is when $\lambda>\hat{\lambda}(\xi)$ but less than $\min (\theta(\xi), 1)$. Here the banker has enough liquidity to serve all $\lambda$ withdrawals. Recall that $\theta(\xi)=(L+\xi I) /\left(D r_{D}\right)$ from (10). Lemma 3 shows that $\hat{\lambda}(\xi)<\theta(\xi)$. So a patient saver's payoff differential is $-D r_{D}$. . 
Finally, if $\min (\theta(\xi), 1)<\lambda \leq 1$, the bank cannot fully repay all savers that withdraw, and is liquidated. A saver gets zero if she waits, while she is repaid with probability $\theta(\xi) / \lambda$ if she withdraws and the expected payoff is $\theta(\xi) / \lambda D r_{D}=(L+\xi I) / \lambda$. Hence, the expected payoff differential is equal to $-(L+\xi I) / \lambda$. Below we refer to this region of $\lambda^{\prime} s$ as the full-run region. ${ }^{9}$

Lemma 3. Strategic complementarities and monitoring. $\delta \leq \hat{\lambda}(\xi)<\theta(\xi)$ for $\xi \in\left[\xi_{l d}, \xi_{u d}\right)$.

Proof. $\theta(\xi)$ and $\hat{\lambda}(\xi)$ are increasing in $\xi$, the latter from Lemma 2. Thus, $\hat{\lambda}(\xi) \geq \delta$ with strict inequality for $\xi>\xi_{l d}$. Moreover, from the definitions of $\theta(\xi)$ and $\hat{\lambda}(\xi)$ in (10) and (19), $\theta(\xi) \geq 1$ and $\hat{\lambda}(\xi)<1$ for $\xi \in\left[\hat{\xi}, \xi_{u d}\right)$. For $\xi \in\left[\xi_{l d}, \hat{\xi}\right), \hat{\lambda}(\xi)<\theta(\xi)$ can be re-written as

$$
\theta(\xi) \frac{\xi R_{D}}{R_{I} r_{D}-\xi R_{D}}<\frac{\xi\left(R_{D}+X /(\omega D)\right)}{R_{I} r_{D}-\xi R_{D}}
$$

which is always true because $\theta(\xi)<1$ for $\xi \in\left[\xi_{l d}, \hat{\xi}\right)$, while $R_{I} r_{D}-\xi R_{D}>0$ from Lemma 2 .

Lemma 3 says that an individual saver may still want to withdraw, even if the bank has enough liquidity to serve all withdrawals, should she believe that the banker will not engage in monitoring.

Step 3. Because the sign of $v(\xi, \lambda)$ in (18) depends on beliefs about $\lambda$, a coordination problem among patient savers arises leading to multiple equilibria. To resolve the multiplicity, we model a global game whereby each patient saver $i$ receives a private noisy signal $x_{i}=\xi+\varepsilon_{i}$ at $t=2 ; \varepsilon_{i}$ are small error terms that are independent and uniformly distributed over $[-\varepsilon, \varepsilon]$. These signals not only provide information about $\xi$, but also about other savers' signals, so that inference about their actions is possible. We seek a symmetric equilibrium characterized by a threshold signal $x^{*}$ such that an individual patient saver will withdraw her deposits if $x_{i}<x^{*}$. Under such a threshold strategy, the number of savers that withdraw at a given level of fundamentals $\xi$ is

$$
\lambda\left(\xi, x^{*}\right)=\left\{\begin{array}{cl}
1 & \text { if } \xi<x^{*}-\varepsilon \\
\delta+(1-\delta) \operatorname{Prob}\left(x_{i} \leq x^{*}\right) & \text { if } x^{*}-\varepsilon \leq \xi \leq x^{*}+\varepsilon . \\
\delta & \text { if } \xi>x^{*}+\varepsilon
\end{array}\right.
$$

If $\xi$ is lower than $x^{*}-\varepsilon$, then all patient savers receive signals $x_{i}<x^{*}$ and withdraw, i.e., $\lambda\left(\xi, x^{*}\right)=1$. The opposite is true for $\xi>x^{*}+\varepsilon$. In this case, all patient savers receive signals $x_{i}>x^{*}$ and keep their deposits in the bank, while only the impatient ones withdraw, i.e., $\lambda\left(\xi, x^{*}\right)=\delta$. Finally, if $\xi \in\left[x^{*}-\varepsilon, x^{*}+\varepsilon\right]$, some patient savers will receive signals that are lower than $x^{*}$ and will withdraw their deposits; others will receive a signal higher than $x^{*}$ and will keep their deposits in the bank. Because $\varepsilon_{i}$ is independently and identically distributed, the law of large numbers obtains and the number of savers withdrawing for a given level of $\xi$ is $\lambda\left(\xi, x^{*}\right)=\delta+(1-\delta) \operatorname{Prob}\left(x_{i} \leq x^{*}\right)=$ $\delta+(1-\delta)\left(x^{*}-\xi+\varepsilon\right) / 2 \varepsilon$, which is increasing in $x^{*}$ and decreasing in $\xi$.

\footnotetext{
${ }^{9}$ For completeness, note that this region will not exist if $\xi \in\left[\hat{\xi}, \xi_{u d}\right)$.
} 
Given $x^{*}$, there are two cutoffs for fundamentals $\xi_{\theta}\left(x^{*}\right)$ and $\xi_{\hat{\lambda}}\left(x^{*}\right)$ such that the bank can serve all withdrawals for realizations $\xi \geq \xi_{\theta}\left(x^{*}\right)$ and the banker has incentives to monitor for realizations $\xi \geq \xi_{\hat{\lambda}}\left(x^{*}\right)$. These two cutoffs are, respectively, the solutions to $\lambda\left(\xi_{\theta}\left(x^{*}\right), x^{*}\right)=\theta\left(\xi_{\theta}\left(x^{*}\right)\right)$ and $\lambda\left(\xi_{\hat{\lambda}}\left(x^{*}\right), x^{*}\right)=\hat{\lambda}\left(\xi_{\hat{\lambda}}\left(x^{*}\right)\right)$, the properties of which are established in Lemma 4 .

Lemma 4. Regions of $\xi$ with illiquidity and no monitoring. Consider signal threshold $x^{*}$. Then, there exist unique cutoffs $\xi_{\theta}\left(x^{*}\right)$ and $\xi_{\hat{\lambda}}\left(x^{*}\right)$, with $\xi_{\theta}\left(x^{*}\right) \leq \xi_{\hat{\lambda}}\left(x^{*}\right)$.

Proof. First for $x^{*} \in\left[\xi_{l d}+\varepsilon, \xi_{u d}-\varepsilon\right)$, all $\xi$ lie in $\left[\xi_{l d}, \xi_{u d}\right)$. If $\xi \in\left[\xi_{l d}, \hat{\xi}\right)$, then using (10) and (21), $\lambda\left(\xi_{\theta}\left(x^{*}\right), x^{*}\right)=\theta\left(\xi_{\theta}\left(x^{*}\right)\right)$ yields

$$
\xi_{\theta}\left(x^{*}\right)=\frac{\varepsilon\left[(1+\delta) D r_{D}-2 \cdot L\right]+x^{*}(1-\delta) D r_{D}}{2 \varepsilon I+(1-\delta) D r_{D}},
$$

which lies in $\left(x^{*}-\varepsilon, x^{*}+\varepsilon\right)$ because: (i) $\lambda\left(\xi, x^{*}\right)$ is continuous and decreasing in $\xi$, (ii) $\theta(\xi)$ is continuous and increasing in $\xi$, (iii) $\lambda\left(x^{*}-\varepsilon, x^{*}\right)=1>\theta\left(x^{*}-\varepsilon\right)$ for $x^{*}<\hat{\xi}-\varepsilon$, and (iv) $\lambda\left(x^{*}+\right.$ $\left.\varepsilon, x^{*}\right)=\delta<\theta\left(x^{*}+\varepsilon\right)$ for $x^{*}>\xi_{l d}+\varepsilon$. For $\xi \in\left[\hat{\xi}, \xi_{u d}\right), \theta(\xi) \geq 1$, so we set $\xi_{\theta}\left(x^{*}\right)=x^{*}-\varepsilon$, i.e., the full-run region is the null set. Similarly, $\xi_{\hat{\lambda}}\left(x^{*}\right)$ is given by $\lambda\left(\xi_{\hat{\lambda}}\left(x^{*}\right), x^{*}\right)=\hat{\lambda}\left(\xi_{\hat{\lambda}}\left(x^{*}\right)\right)$, which yields

$$
\delta+(1-\delta) \frac{x^{*}+\varepsilon-\xi_{\hat{\lambda}}\left(x^{*}\right)}{2 \varepsilon}=\frac{\left(\xi_{\hat{\lambda}}\left(x^{*}\right) I+L\right) R_{I}-\xi_{\hat{\lambda}}\left(x^{*}\right)\left(D R_{D}+X / \omega\right)}{D\left(R_{I} r_{D}-\xi_{\hat{\lambda}}\left(x^{*}\right) R_{D}\right)}
$$

Though (23) is quadratic, there exists a unique $\xi_{\hat{\lambda}}\left(x^{*}\right)$ lying in $\left(x^{*}-\varepsilon, x^{*}+\varepsilon\right)$. First, $\lambda\left(\xi, x^{*}\right)$ is continuous and decreasing in $\xi$, while $\hat{\lambda}(\xi)$ is continuous and increasing in $\xi$ due to Lemma 2 . Second, $\lambda\left(x^{*}-\varepsilon, x^{*}\right)=1>\hat{\lambda}\left(x^{*}-\varepsilon\right)$ for $x^{*}<\xi_{u d}-\varepsilon$, and third, $\lambda\left(x^{*}+\varepsilon, x^{*}\right)=\delta<\hat{\lambda}\left(x^{*}+\varepsilon\right)$ for $x^{*}>\xi_{l d}+\varepsilon$. So, the two functions cross once in $\left(x^{*}-\varepsilon, x^{*}+\varepsilon\right)$ and there exists a unique $\xi_{\hat{\lambda}}\left(x^{*}\right)$. Because $\hat{\lambda}(\xi)<\theta(\xi)$ for any $\xi \in\left[\xi_{l d}, \xi_{u d}\right)$ from Lemma 3, we get that $\xi_{\theta}\left(x^{*}\right)<\xi_{\hat{\lambda}}\left(x^{*}\right)$.

To conclude, for $x^{*}$ such that $\xi<\xi_{l d}$, we have $\lambda\left(\xi, x^{*}\right)=1$ and, thus, set $\xi_{\theta}\left(x^{*}\right)=\xi_{\hat{\lambda}}\left(x^{*}\right)=$ $x^{*}+\varepsilon$. For $x^{*}$ such that $\xi>\xi_{u d}$, we have $\lambda\left(\xi, x^{*}\right)=\delta$ and set $\xi_{\theta}\left(x^{*}\right)=\xi_{\hat{\lambda}}\left(x^{*}\right)=x^{*}-\varepsilon$.

Under threshold strategy $x^{*}$, the beliefs of an individual saver about aggregate withdrawals are well-defined, allowing her to compute the expected payoff differential between waiting and withdrawing given her private signal. The saver uses the signal to update her beliefs about the realization of $\xi$. Given that both $\xi$ and $\varepsilon_{i}$ are uniformly distributed, the posterior distribution of $\xi$ given $x_{i}$ is $\xi \mid x_{i} \sim U\left[x_{i}-\varepsilon, x_{i}+\varepsilon\right]$. Thus, the expected payoff differential is

$$
\Delta\left(x_{i}, x^{*}\right)=\frac{1}{2 \varepsilon} \int_{x_{i}-\varepsilon}^{x_{i}+\varepsilon} v\left(\xi, \lambda\left(\xi, x^{*}\right)\right) d \xi .
$$

A patient saver prefers to withdraw if $\Delta\left(x_{i}, x^{*}\right)<0$, for all $x_{i}<x^{*}$, and prefers to wait if $\Delta\left(x_{i}, x^{*}\right)>$ 0 , for all $x_{i}>x^{*} . \Delta\left(x_{i}, x^{*}\right)$ is continuous in $x_{i}$, because a change in the signal only changes the limits of integration $\left[x_{i}-\varepsilon, x_{i}+\varepsilon\right]$ and the integrand is bounded. Hence, a patient saver who receives signal 
$x_{i}=x^{*}$ is indifferent between waiting and withdrawing if

$$
\bar{\Delta}\left(x^{*}\right) \equiv \Delta\left(x^{*}, x^{*}\right)=\frac{1}{2 \varepsilon} \int_{x^{*}-\varepsilon}^{x^{*}+\varepsilon} v\left(\xi, \lambda\left(\xi, x^{*}\right)\right) d \xi=0 .
$$

We need to establish two results. First, that a threshold strategy is an equilibrium, i.e., $\Delta\left(x_{i}, x^{*}\right)<$ 0 for $x_{i}<x^{*}$ and $\Delta\left(x_{i}, x^{*}\right)>0$ for $x_{i}>x^{*}$. Second, and more importantly, that there exists a unique $x^{*}$ satisfying $\bar{\Delta}\left(x^{*}\right)=0$. The proof of the first result is as follows.

Step 4. For signal $x_{i}<x^{*}$, decompose the intervals $\left[x_{i}-\varepsilon, x_{i}+\varepsilon\right]$ and $\left[x^{*}-\varepsilon, x^{*}+\varepsilon\right]$ into a common part $c=\left[x_{i}-\varepsilon, x_{i}+\varepsilon\right] \cap\left[x^{*}-\varepsilon, x^{*}+\varepsilon\right]$, and two disjoint parts $d^{i}=\left[x_{i}-\varepsilon, x_{i}+\varepsilon\right] \backslash c$ and $d^{*}=\left[x^{*}-\varepsilon, x^{*}+\varepsilon\right] \backslash c$. Under a common threshold strategy, all savers form beliefs according to (21). Individual signals matter for savers' posterior beliefs about $\xi$ and the possible realizations of $\lambda$. Hence, (24) and (25) can be also decomposed to common and disjoint parts, respectively, as $\Delta\left(x_{i}, x^{*}\right)=\Delta_{\xi \in c}+\Delta_{\xi \in d^{i}}$ and $\bar{\Delta}\left(x^{*}\right)=\Delta_{\xi \in c}+\Delta_{\xi \in d^{*}}$. From (21), $\lambda$ is always one over $d^{i}$, thus $\Delta_{\xi \in d^{i}}=\int_{\xi \in d^{i}} v(\xi, 1) d \xi=-\int_{\xi \in d^{i}}(\xi I+L) d \xi<0$ from (18). Hence, it suffices to show that $\Delta_{\xi \in c}<0$. We will use the facts that $\bar{\Delta}\left(x^{*}\right)=0$, and that $v$ changes sign ("crosses zero") only once, while being positive and negative for higher and lower values of $\xi \in\left[x^{*}-\varepsilon, x^{*}+\varepsilon\right]$. Hence, $\Delta_{\xi \in d^{*}}>0$ and $\Delta_{\xi \in c}<0$, since the fundamentals are higher over $d^{*}$ than $c$. The fact that $v$ may be increasing in $\xi$ in the lower segment of $c$ does not matter, because $v$ is still negative in that segment. Essentially, observing a signal $x_{i}$ below $x^{*}$ shifts probability from positive values of $v$ to negative values of $v$ -recall that noise is uniformly distributed- and $\Delta\left(x_{i}, x^{*}\right)<\bar{\Delta}\left(x^{*}\right)=0$. The argument holds trivially if the interval $c$ is empty. The proof for $x_{i}>x^{*}$ is similar. As in Goldstein and Pauzner (2005), single crossing of $v$ at zero suffices for a threshold strategy, should it exist, to be an equilibrium.

Step 5. To prove existence, note that $\bar{\Delta}\left(x^{*}\right)<0$ for $x^{*} \leq \xi_{l d}-\varepsilon$, because this level of signal $x^{*}$ implies that $\xi \leq \xi_{l d}$ and, thus, $v\left(\xi, \lambda\left(\xi, x^{*}\right)\right)<0$ always. Similarly, $\bar{\Delta}\left(x^{*}\right)>0$ for $x^{*} \geq \xi_{\text {ud }}+\varepsilon$. Moreover, all the integrands and the limits of integration in $\bar{\Delta}\left(x^{*}\right)$ are bounded and continuous in $x^{*}$. Hence, $\bar{\Delta}\left(x^{*}\right)$ is continuous in $x^{*}$, which implies there are solutions to $\bar{\Delta}\left(x^{*}\right)=0$ given the existence of $\xi_{l d}$ and $\xi_{u d}$ for Lemmas 1 and 2.

To prove that $x^{*}$ is unique, it is typical in the global games literature to consider environments in which state monotonicity holds (Morris and Shin, 2003, Rochet and Vives, 2004, Goldstein and Pauzner, 2005). This property requires that $\bar{\Delta}\left(x^{*}\right)$ is always increasing in $x^{*}$, which implies that the solution to $\bar{\Delta}\left(x^{*}\right)=0$ is unique since $\bar{\Delta}$ can only cross zero once. However, this property does not necessarily hold in our model, because as we show in detail below, $v(\xi, \lambda)$ is decreasing in $\xi$ in the full-run region. This means we cannot ensure that $\bar{\Delta}$ is always increasing. The ambiguity arises because savers have higher incentives to run on a stronger bank than a weaker bank, conditional on a run occurring, because their chances of getting paid are higher. We call this property perverse state monotonicity. To see the issue, use Lemma (4) to write $\bar{\Delta}\left(x^{*}\right)$ as

$$
\bar{\Delta}\left(x^{*}\right)=-\int_{x^{*}-\varepsilon}^{\xi_{\theta}\left(x^{*}\right)} \frac{\xi I+L}{\lambda\left(\xi, x^{*}\right)} \frac{d \xi}{2 \varepsilon}-\int_{\xi_{\theta}\left(x^{*}\right)}^{\xi_{\hat{\lambda}}\left(x^{*}\right)} \operatorname{Dr}_{D} \frac{d \xi}{2 \varepsilon}+\int_{\xi_{\hat{\lambda}}\left(x^{*}\right)}^{x^{*}+\varepsilon}\left(\omega D R_{D}-D r_{D}\right) \frac{d \xi}{2 \varepsilon} .
$$


Varying $x^{*}$ changes both the threshold strategy and the signal that the threshold saver receives, changing both the limits of integration and $\lambda\left(\xi, x^{*}\right)$ in (26). It is convenient to set $x^{*}=\tilde{x}+\Delta x$ and vary $\Delta x$, while keeping $\tilde{x}$ fixed. Defining $\tilde{\xi}=\xi-\Delta x$, we can express $\bar{\Delta}\left(x^{*}\right)$ as $\bar{\Delta}(\tilde{x}+\Delta x)$ equal to

$$
-\int_{\tilde{x}-\varepsilon}^{\xi_{\theta}(\tilde{x}+\Delta x)-\Delta x} \frac{(\tilde{\xi}+\Delta x) I+L}{\lambda(\tilde{\xi}, \tilde{x})} \frac{d \tilde{\xi}}{2 \varepsilon}-\int_{\xi_{\theta}(\tilde{x}+\Delta x)-\Delta x}^{\xi_{\hat{\lambda}}(\tilde{x}+\Delta x)-\Delta x} D r_{D} \frac{d \tilde{\xi}}{2 \varepsilon}+\int_{\xi_{\hat{\lambda}}(\tilde{x}+\Delta x)-\Delta x}^{\tilde{x}+\varepsilon}\left(\omega D R_{D}-D r_{D}\right) \frac{d \tilde{\xi}}{2 \varepsilon},
$$

where we have used the fact that $\lambda(\tilde{\xi}+\Delta x, \tilde{x}+\Delta x)=\lambda(\tilde{\xi}, \tilde{x})$, i.e., perceived withdrawals are unaffected by varying $\Delta x$, as a higher threshold means that the threshold saver also has higher posterior over $\xi$. The transformation in (27) shows that increasing $\Delta x$ is equivalent to having the threshold saver think that the liquidation value is higher by an amount $\Delta x$ for all values $\xi$ in the interval $[\tilde{x}-\varepsilon, \tilde{x}+\varepsilon]$. This enables the easy comparison of $\bar{\Delta}(\tilde{x}+\Delta x)$ for liquidation values in the same interval $[\tilde{x}-\varepsilon, \tilde{x}+\varepsilon]$ but for different values of $\Delta x$ given by

$$
\frac{d \bar{\Delta}(\tilde{x}+\Delta x)}{d \Delta x}=-\int_{\tilde{x}-\varepsilon}^{\xi_{\hat{\theta}}(\tilde{x}+\Delta x)-\Delta x} \frac{I}{\lambda(\tilde{\xi}, \tilde{x})} \frac{d \tilde{\xi}}{2 \varepsilon}+\frac{1}{2 \varepsilon}\left[1-\frac{d \xi_{\hat{\lambda}}(\tilde{x}+\Delta x)}{d \Delta x}\right] \omega D R_{D} .
$$

Note that the limit of integration, $\xi_{\theta}(\tilde{x}+\Delta x)-\Delta x$, also changes with $\Delta x$, but it does not affect $\bar{\Delta}(\tilde{x}+\Delta x)$ because $((\tilde{\xi}+\Delta x) I+L) / \lambda(\tilde{\xi}, \tilde{x})$ evaluated at $\tilde{\xi}=\xi_{\theta}(\tilde{x}+\Delta x)-\Delta x$ is equal to $D r_{D}$ using $\lambda\left(\xi_{\theta}(\tilde{x}+\Delta x)-\Delta x, \tilde{x}\right)=\lambda\left(\xi_{\theta}(\tilde{x}+\Delta x), \tilde{x}+\Delta x\right)=\theta\left(\xi_{\theta}(\tilde{x}+\Delta x)\right)=\left(\tilde{\xi}_{\hat{\theta}}(\tilde{x}+\Delta x) I+L\right) /\left(D r_{D}\right)$.

Thus, the overall effect on $\bar{\Delta}$ is ambiguous. A higher threshold signal pushes down the payoff differential in the full-run region (first term in (28)), but it increases region where monitoring occurs (second term in (28)). To see that the latter term is positive, totally differentiate $\lambda\left(\xi_{\hat{\lambda}}(\tilde{x}+\right.$ $\Delta x), \tilde{x}+\Delta x)=\hat{\lambda}\left(\xi_{\hat{\lambda}}(\tilde{x}+\Delta x)\right)$, which is the condition that gives $\xi_{\hat{\lambda}}(\tilde{x}+\Delta x)$. Doing so yields $d \xi_{\hat{\lambda}}(\tilde{x}+$ $\Delta x) / d \Delta x=\partial \lambda / \partial \Delta x \cdot(d \hat{\lambda} / d \xi-\partial \lambda / \partial d \xi)^{-1}$, which is less that one because $\partial \lambda / \partial \Delta x=\partial \lambda / \partial x^{*}=$ $-\partial \lambda / \partial d \xi>0$ from (21) and $d \hat{\lambda} / d \xi>0$ from Lemma 2 .

Because of the perverse state monotonicity, we need to use a new proof that covers three possible cases that are depicted in Figure 3. A first possibility is similar to what is typically studied in the literature where $v(\xi, \lambda)$ is always increasing in the state. If this happens to be true, the threshold solving $\bar{\Delta}\left(x^{*}\right)=0$ will be unique as depicted in the left panel in Figure 3. However, $v(\xi, \lambda)$ may not be increasing in the state. Instead, it could be a situation, as in the middle panel, where there are multiple solutions, or a situation, as in the right panel, where there is a unique solution, despite the fact that $\bar{\Delta}\left(x^{*}\right)$ is not increasing in $x^{*}$ everywhere in the domain.

To establish uniqueness under perverse state monotonicity, notice that, to rule out the problematic middle case, $\bar{\Delta}\left(x^{*}\right)$ does not need to be increasing for any $x^{*}$ (as is typically required) but instead only needs to be strictly increasing at candidate solutions that solve $\bar{\Delta}\left(x^{*}\right)=0$, which we know exist. This weaker requirement is graphically depicted in Figure 4. In the left panel, where there are multiple solutions, $\bar{\Delta}$ will necessarily cross the $\mathrm{x}$-axis both from below and from above. This means that the derivative of $\bar{\Delta}$ at the candidate solutions, which are depicted by the dots on the x-axis, can be either positive or negative. On the contrary, in the unique solution in the right 


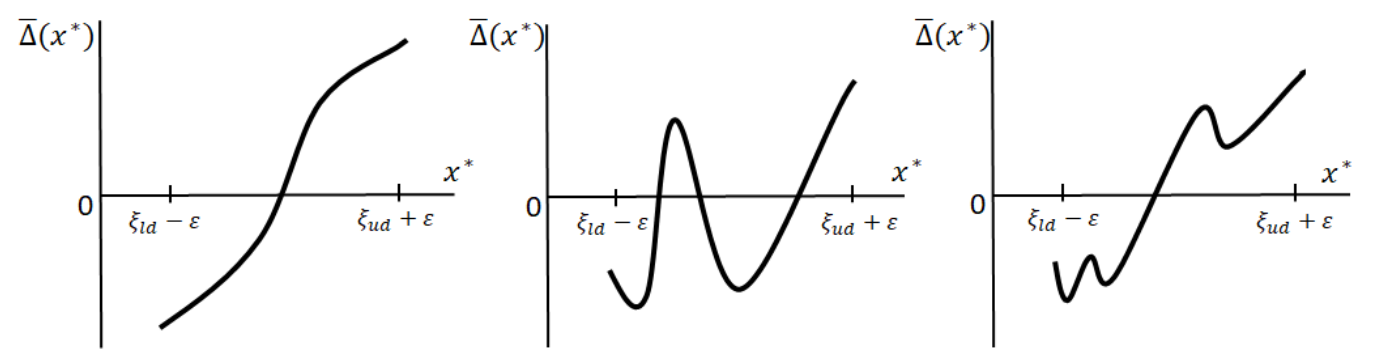

Figure 3: Perverse State Monotonicity

panel, the derivative at the candidate solution is strictly positive. Hence, the strategy to establish uniqueness requires showing that the derivative of $\bar{\Delta}$ at a solution is always strictly positive.
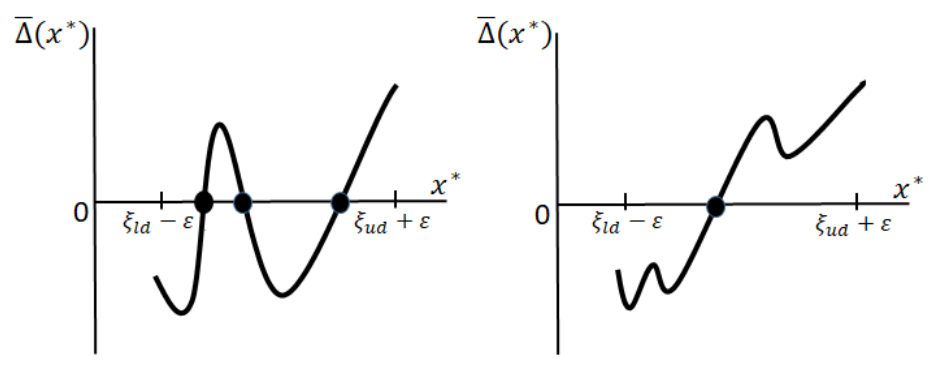

Figure 4: Graphical Representation of Uniqueness Argument

We analyze the equilibrium, as the noise shrinks to zero, $\varepsilon \rightarrow 0$. Note that taking the limit $\varepsilon \rightarrow 0$ and using the expressions in Lemma 4 , the thresholds for fundamentals $\xi_{\theta}\left(x^{*}\right)$ and $\xi_{\hat{\lambda}}\left(x^{*}\right)$ converge to the same threshold $\xi^{*}$ equal to $x^{*}$. When working with $\varepsilon \rightarrow 0$, it is convenient to change the variable of integration in $\bar{\Delta}\left(x^{*}\right)$ from $\xi$ to $\lambda$. Recall that a saver who receives signal $x^{*}$ believes that $\lambda$ is given by $\lambda\left(\xi, x^{*}\right)=\delta+(1-\delta)\left(x^{*}-\xi+\varepsilon\right) / 2 \varepsilon$ and that $\xi \sim U\left[x^{*}-\varepsilon, x^{*}+\varepsilon\right]$. Thus, as $\xi$ increases uniformly from $x^{*}-\varepsilon$ to $x^{*}+\varepsilon, \lambda$ decreases uniformly from 1 to $\delta$. Given $\xi$, define

$$
\begin{aligned}
\Delta_{w t}(\xi) & =\int_{\delta}^{\hat{\lambda}(\xi)} \omega D R_{D} \frac{d \lambda}{1-\delta} \\
\Delta_{w d}(\xi) & =\int_{\delta}^{\theta(\xi)} D r_{D} \frac{d \lambda}{1-\delta}+\int_{\theta(\xi)}^{1} \frac{\xi I+L}{\lambda} \frac{d \lambda}{1-\delta}
\end{aligned}
$$

where, for completeness, we recall equations (10) and (19),

$$
\theta(\xi)=\frac{\xi I+L}{D r_{D}} \quad \text { and } \quad \hat{\lambda}(\xi)=\frac{(\xi I+L) R_{I}-\xi\left(D R_{D}+X / \omega\right)}{D\left(R_{I} r_{D}-D\right)}
$$

Rewrite $\xi=x^{*}+\varepsilon-2 \varepsilon(\lambda-\delta) /(1-\delta)$ as a function of $\lambda \sim U[\delta, 1]$. Per change of variable of integration in (25) from $\xi$ to $\lambda$ and examination of $v(\xi, \lambda)$ in (18), one can see that $\Delta_{w t}\left(\xi^{*}\right)$ is the payoff from waiting, while $\Delta_{w d}\left(\xi^{*}\right)$ is the payoff from withdrawing for a patient saver when $\xi=\xi^{*}$. 
The indifference condition (25) at the equilibrium $\xi^{*}$ can be written, for $\varepsilon \rightarrow 0$, as

$$
\Delta_{w t}\left(\xi^{*}\right)=\Delta_{w d}\left(\xi^{*}\right)
$$

The following Proposition establishes the key result.

Proposition 1. Threshold uniqueness. The solution $\xi^{*}$ to (29) is unique.

To prove the proposition, we compare the elasticies of $\Delta_{w t}$ and $\Delta_{w d}$ at $\xi=\xi^{*}$. Define and calculate the elasticities as

$$
\begin{aligned}
\eta_{w t}(\xi) & =\Delta_{w t}^{\prime}(\xi) \frac{\xi}{\Delta_{w t}(\xi)}=\hat{\lambda}^{\prime}(\xi) \frac{\xi}{\hat{\lambda}(\xi)-\delta} \\
\eta_{w d}(\xi) & =\Delta_{w d}^{\prime}(\xi) \frac{\xi}{\Delta_{w d}(\xi)}=\frac{\int_{\theta(\xi)}^{1}\left(\frac{\xi I}{\lambda} \frac{d \lambda}{1-\delta}\right.}{\Delta_{w d}(\xi)}
\end{aligned}
$$

where we have used that $D r_{D}=(\xi I+L) / \theta(\xi)$ from the definition of $\theta(\xi)$ and therefore the derivatives with respect to the integral bounds cancel. We shall show that

$$
\eta_{w t}\left(\xi^{*}\right)>1>\eta_{w d}\left(\xi^{*}\right)
$$

With that and (29), $\Delta_{w t}^{\prime}\left(\xi^{*}\right)>\Delta_{w d}^{\prime}\left(\xi^{*}\right)$, i.e., the difference $\Delta_{w t}(\xi)-\Delta_{w d}(\xi)$ has a positive slope at $\xi=\xi^{*}$ and can cross zero only from below, establishing the proposition.

Equation (30) will be established using Lemmas 1 and 2 as well as the next two Lemmas.

Lemma 5. Elasticity of payoff from waiting. Given Lemmas 1 and $2, \hat{\lambda}^{\prime}(\xi)>(\hat{\lambda}(\xi)-\delta) / \xi$.

Proof. Using the definition of $\hat{\lambda}(\xi)$ and after some algebra, we get

$$
\hat{\lambda}^{\prime}(\xi)=\frac{\hat{\lambda}(\xi)-\delta}{\xi}\left(1+\frac{\xi R_{D}}{R_{I} r_{D}-\xi R_{D}}\right)+\frac{\left(\delta D r_{D}-L\right) R_{I}}{\xi D\left(R_{I} r_{D}-\xi R_{D}\right)}>\frac{\hat{\lambda}(\xi)-\delta}{\xi},
$$

because $\delta D r_{D}-L>0$ from Lemma 1 , and $R_{I} r_{D}-\xi R_{D}>0$ for $\xi \leq \bar{\xi}$ from Lemma 2.

The lemma establishes that $\eta_{w t}\left(\xi^{*}\right)>1$, the left part of (30). This is an intuitive property, because it implies that a saver believes that the probability of monitoring, given by $(\hat{\lambda}(\xi)-\delta) /(1-$ $\delta$ ), would increase by more than one percent if she received a one percent higher signal about $\xi$. This is because a better $\xi$ reduces the need to recall loans to pay impatient savers -the second term of $\hat{\lambda}^{\prime}(\xi)$ in (31)- and the loans that are left in place can serve even more withdrawals by patient savers if needed-the first term of $\hat{\lambda}^{\prime}(\xi)$ in (31).

\section{Lemma 6. Elasticity of payoff from withdrawing.}

$$
\int_{\theta(\xi)}^{1} \frac{\xi I}{\lambda} \frac{d \lambda}{1-\delta}<\Delta_{w d}(\xi)
$$


Proof. This follows, since the term on the left is one of the summands defining $\Delta_{w d}(\xi)$, and since all the summands are nonnegative, with at least one strictly positive.

This lemma establishes that $1>\eta_{w d}\left(\xi^{*}\right)$, the right part of (30). This is intuitive, because a part of $\Delta_{w d}(\xi)$ is linearly increasing in $\xi$ (in the full run region) and another part is positive but independent of $\xi$ (in the region where deposit withdrawals are paid in full). So raising $\xi$ increases the payoff from withdrawing by less than one for one. Hence, Proposition 1 is now established.

Step 6. Consequently, we have the following Proposition.

Proposition 2. Threshold-strategy equilibrium. Assume that the model parameters are such that Lemmas 1 and 2 obtain. For $\varepsilon \rightarrow 0$, there exists unique $\xi^{*}$ such that a patient depositor does not withdraw at $t=2$ if $x_{i}>\xi^{*}$, and withdraws if $x_{i}<\xi^{*}$. Therefore, a run does not occur if $\xi \geq \xi^{*}$, and the bank is fully liquidated if $\xi<\xi^{*}$.

Proof. Given lower and upper dominance regions from Lemmas 1 and 2, Proposition 1 shows that there exists a unique $\xi^{*}$ that the threshold signal $x^{*}$ and threshold for fundamentals, $\xi_{\theta}\left(x^{*}\right)$ and $\xi_{\hat{\lambda}}\left(x^{*}\right)$, converge to for $\varepsilon \rightarrow 0$. Moreover, we showed earlier that a threshold strategy, whereby an individual patient saver withdraws only if $x_{i}$ is below a common $x^{*}$, is an equilibrium.

In the rest of paper, we use the following notation for the condition that yields $\xi^{*}$, as a function of all other, pre-determined, endogenous variables:

$$
G G \equiv \lim _{\varepsilon \rightarrow 0} \bar{\Delta}\left(x^{*}\right)=\Delta_{w t}\left(\xi^{*}\right)-\Delta_{w d}\left(\xi^{*}\right)=0 .
$$

The Online Appendix shows that Proposition 2 is also true for positive, but sufficiently small, $\varepsilon$.

\subsection{Private Equilibrium}

The banker will choose both sides of the balance sheet to maximize her own profits as shown in (13). The choices of loans, liquidity, deposits, and equity need to satisfy the global game constraint (32), which determines the run threshold, the deposit supply schedule (2), which determines the deposit rates, and the loan demand schedule (7), which determines the loan rate. The balance sheet identity also holds so only three of the four variables can be freely chosen. The banker will internalize the effect of her actions on the run threshold, on deposit supply, and on loan demand. As a result, conditions (32), (2), and (7) not only need to be satisfied in equilibrium, but will also be explicit constraints in the banker's optimization problem; the solution to which defines the private equilibrium (PE). Essentially, this means that the banker also chooses directly the level of the run threshold as well as the deposit and loan rates, rather than letting them be determined competitively in equilibrium. Finally, the banker will never choose allocations that result in lower welfare than her outside option. The bank can either invest in the storage technology or lend to entrepreneurs without accepting savers' deposits. Thus, the banker's outside option is $\overline{\mathbb{U}}_{B}=\max \left[\mathbb{U}_{B}^{\alpha}, \mathbb{U}_{B}^{n}\right]$, where 
$\mathbb{U}_{B}^{\alpha}$ is the utility in autarky (where the bank holds only liquid assets) and $\mathbb{U}_{B}^{n}$ is the utility when the banker does not take deposits and lends to $E$ using only her own capital. ${ }^{10}$

Definition 1. The private equilibrium is defined as the set of bank assets, $\{I, L\}$, bank liabilities, $\{D, E\}$, the run threshold, $\xi^{*}$, deposit rates, $\left\{r_{D}, R_{D}\right\}$, and the loan rate, $R_{I}$, that maximize banker's utility, $\mathbb{U}_{B}$, defined in (13) subject to the balance sheet (BS) constraint (9), the global game (GG) constraint (32), the deposit supply (DS) schedule (2), and the loan demand (LD) schedule (7).

Each first-order condition in the private equilibrium takes the form

$$
\frac{\partial \mathbb{U}_{B}}{\partial C}+\sum_{Y} \psi_{Y} \frac{\partial \mathcal{Y}}{\partial C}=0
$$

where $\mathcal{C} \in\left\{I, L, D, E, \xi^{*}, r_{D}, R_{D}, R_{I}\right\}, \psi_{Y}$ are the shadow values on constraints $\mathcal{Y} \in\{B S, G G, D S, L D\}$ and $\partial \mathcal{Y} / \partial \mathcal{C}$ the partial derivatives capturing the effect of choice $C$ on these constraints. Take for example the lending choice, i.e., $C=I$, then (33) says that the optimal level of lending is determined by having the banker trade off the marginal return accruing to her, $\partial \mathbb{U}_{B} / \partial I$, against the shadow cost of funds, $\psi_{B S}$, and the way it affects the run threshold determination, the deposit supply, and the loan demand. The optimality condition for the other variables can be similarly interpreted, and we report the detailed expressions for the partial derivatives in the Online Appendix.

The optimality condition with respect to $E$, which is $\psi_{B S}=W^{\prime}\left(e_{B}-E\right)$, deserves special attention because it highlights another way in which our model differs from others in the literature. This condition says that injecting more equity requires the banker to give up consumption in the initial period in exchange for increasing the funds of the bank. ${ }^{11}$ Thus, the shadow cost of funds, $\psi_{B S}$, is inversely related to the amount of equity the banker puts in the bank. In banking models without endogenous credit and run-risk, the higher funding costs of injecting more equity would feed in higher loan rates and lower investment. In our model, this is not necessarily true: Higher equity can improve the safety of deposits, which can be compatible with lower loan rates and more investment.

While the banker is free to choose any three of the quantities on her balance sheet, to explain how the model works, it is helpful to summarize the choices in terms of three different combinations. In particular, we will summarize the private equilibrium in terms of the asset allocation choice, the capital structure choice, and the level of lending (that is deposit funded). The three margins can be easily interpreted (see the Online Appendix for detailed expressions).

\footnotetext{
${ }^{10}$ The utility in autarky is $\mathbb{U}_{B}^{\alpha}=W\left(e_{B}-\alpha\right)+\alpha$, where $\alpha>0$ is the storage investment satisfying $W^{\prime}\left(e_{B}-\alpha\right)=1$. Note that, if $W^{\prime}\left(e_{B}\right)>1$, then $\alpha=0$. If the banker lends to entrepreneurs using only her own capital, her utility is $\mathbb{U}_{B}^{n}=W\left(e_{B}-n\right)+\omega\left(1+r_{n}\right) n-X$, where $r_{n}$ is the interest rate determined by the loan demand $1+r_{n}=A-c^{\prime}(n) / \omega$. Because $B$ internalizes how the choice of $n$ affects the loan rate that entrepreneurs are willing to accept, the optimal $n>0$ is the solution to $-W^{\prime}\left(e_{B}-n\right)+\omega A-c^{\prime}(n)-c^{\prime \prime}(n) n=0$.

${ }^{11}$ Note that the condition does not include term for the effect of additional equity on constraints $G G, D S$, and $L D$. This is true because, E does not appear directly in (32), (2), or (7), but this does not mean that equity is irrelevant for their determination. On the contrary, equity issuance can affect the run probability, the deposit supply, and the loan demand through its joint determination with other equilibrium variables.
} 
The asset allocation margin is

$$
A A M_{P E}=\left(\frac{d \mathbb{U}_{B}}{d L}-\frac{d \mathbb{U}_{B}}{d I}\right)+\frac{d \mathbb{U}_{B}}{d \xi^{*}}\left(\frac{d \xi^{*}}{d L}-\frac{d \xi^{*}}{d I}\right)
$$

$A A M_{P E}$ captures the decision to shift a unit of loans into liquid assets, consisting of the effect on $B$ 's utility via bank profitability (the first term) and the effect via the run probability (the second term).

Similarly, the capital structure margin is

$$
C S M_{P E}=\left(\frac{d \mathbb{U}_{B}}{d E}-\frac{d \mathbb{U}_{B}}{d D}\right)-\frac{d \mathbb{U}_{B}}{d \xi^{*}} \frac{d \xi^{*}}{d D}
$$

and captures the decision to replace a unit of deposits with equity.

Lastly, the deposit lending margin is

$$
D I M_{P E}=\left(\frac{d \mathbb{U}_{B}}{d I}+\frac{d \mathbb{U}_{B}}{d D}\right)+\frac{d \mathbb{U}_{B}}{d \xi^{*}}\left(\frac{d \xi^{*}}{d I}+\frac{d \xi^{*}}{d D}\right)
$$

and captures the decision to raise a unit of deposits in order to expand lending. This decision will raise run-risk because both sides of the balance sheet become riskier, and the only reason to do it will be to make more credit available to the entrepreneur. So this margin is one way to describe how the banker's private choices affect the entrepreneur relative to the saver.

To understand how these margins determine the structure of the bank's balance sheet, start in reverse order. Given a level of $D$ and $L, D I M_{P E}$ determines the level of lending by fixing the spread between loans and deposits; higher $I$ requires a lower spread, all else being equal, and vice versa. Then, $A A M_{P E}$ and $C S M_{P E}$ simultaneously fix $L$ and $D$, or the liquidity ratio $\ell=L /(I+L)$ and the leverage ratio $k=E /(E+D)$, given that $E=I+L-D$ from the balance sheet identity.

Corollary 1. The liquidity ratio, $\ell$, the leverage ratio, $k$, and the loan-deposit spread, $R_{I}-R_{D}$, characterize the banker's optimal choices. ${ }^{12}$

Before we compare the private equilibrium and the social one, we briefly discuss the assumptions about the banker's behavior. First, the possibility of a run disciplines the banker, who internalizes how her lending and funding choices affect $\xi^{*}$ and hence the probability that she will make profits (see, also, Calomiris and Kahn, 1991; Diamond and Rajan, 2000). These considerations are captured by the terms multiplied by the shadow value on the global game constraint, $\psi_{G G}$, in (33).

Second, the banker internalizes how her choices affect $r_{D}$ and $R_{D}$. This is possible because the debt is demandable and savers can fully observe the bank's balance sheet. At the moment of entering into a deposit contract, savers observe the balance sheet and can rationally compute the underlying risks. Hence, if the banker alters the balance sheet, savers can request new contract terms, so that their optimal supply of deposits reflects the new risks. The banker anticipates this behavior and

\footnotetext{
${ }^{12}$ The statistics in Corollary 1 are not exclusive. For example, one could combine (33) with respect to $L$ and $D$, on the one hand, and with respect to $E$ and $I$, on the other, to obtain alternative margins to $A A M$ and $C S M$. These margins would be proxied by a reserve ratio, $L / D$, and a risk-weighted capital ratio, $C R=E / I$, respectively.
} 
internalizes the impact of all of her choices on deposit supply. These considerations are captured by the terms multiplied by the shadow value on the deposit supply schedule, $\psi_{D S}$, in (33).

Third, the banker internalizes how all of her choices affect $R_{I}$. These considerations are captured by the terms multiplied by the shadow value on the loan demand schedule, $\psi_{L D}$, in (33).

We relax these assumptions in the extensions in Section 5.

\section{Efficient Allocations}

Our bank offers socially useful services but faces the risk of a run. Run-risk is harmful for all agents in the economy, but the effects on their respective welfare differ. $B$ would benefit from low run-risk, but reducing the risk lowers profits. $S$ would prefer lower run-risk and more deposit services, while $E$ would benefit from higher lending accompanied by lower run-risk.

The private banking choices are socially inefficient. $B$ fully internalizes how her lending and capital decisions change the probability of a run, the deposit rates, and the loan rate. Yet, $B$ maximizes her own utility, disregarding the direct effect of her actions on the welfare of $S$ and $E$. In turn, $S$ and $E$ are atomistic and do not internalize how their own decisions affect the bank (and the probability of a run). To examine how private choices distort outcomes, we consider a social planner who internalizes the effects of banking choices on all agents but is constrained by the market structure of the economy. We show that all three margins derived in section 2.5 are distorted from the planner's perspective. Section 3.1 sets up the planner's problem and identifies the sources of differences between the private and social optimization margins. Section 3.2 presents a numerical solution to the model and describes how the private and social planner's allocations differ.

\subsection{Social Planner}

The social planner maximizes the following social welfare function:

$$
\mathbb{U}_{S P}=\mathbb{U}_{B}+w_{S} \mathbb{U}_{S}+w_{E} \mathbb{U}_{E} .
$$

$w_{S} \geq 0, w_{E} \geq 0$ are the weights assigned to $S$ and $E$, while we have normalized the weight on $B$ to one to facilitate the comparison with the private optimality conditions (other normalizations are also possible). Agents' utilities are given by (1), (5), and (13). The planner is constrained by the market structure, i.e., she cannot use lump-sum transfers to allocate resources across agents or complete any missing markets. ${ }^{13}$ Moreover, the planner respects the run determination given by (32) as well as the deposit supply and loan demand schedules (2) and (7). Given that the latter two hold with equality in the planner's problem, we can substitute the indirect utilities (4) and (8) in (37) to get:

$$
\mathbb{U}_{S P}^{*}=\mathbb{U}_{B}+w_{S} \mathbb{U}_{S}^{*}+w_{E} \mathbb{U}_{E}^{*} .
$$

\footnotetext{
${ }^{13}$ Due to market incompleteness, we cannot unambiguously obtain a welfare criterion to maximize total surplus. Thus, we assign weights for different agents in a social welfare function and study different constellations of these weights.
} 
Using (38) instead of (37) adds a lot of tractability in the optimizing conditions and, as we show below, allows us to pinpoint the sources of distortions in the private equilibrium that a planner would like to address. In particular, $\mathbb{U}_{S}^{*}$ only depends on $D$ and $\xi^{*}$ with

$$
\begin{gathered}
\frac{\partial \mathbb{U}_{S}^{*}}{\partial D}=-U^{\prime \prime}\left(e_{S}-D\right)-(1-q) V^{\prime \prime}\left(D r_{D}\right) D r_{D}^{2}>0, \\
\frac{\partial \mathbb{U}_{S}^{*}}{\partial \xi^{*}}=-\left[V\left(D r_{D}\right)-V^{\prime}\left(D r_{D}\right) D r_{D}\right] \frac{1}{\Delta_{\xi}}<0 .
\end{gathered}
$$

Therefore, a planner who cares primarily about $S$ would like to increase $D$ and reduce $\xi^{*}$.

Similarly, $\mathbb{U}_{E}^{*}$ only depends on $I$ and $\xi^{*}$ with

$$
\begin{gathered}
\frac{\partial \mathbb{U}_{E}^{*}}{\partial I}=(1-q) c^{\prime \prime}(I) I>0, \\
\frac{\partial \mathbb{U}_{E}^{*}}{\partial \xi^{*}}=-\left[c^{\prime}(I) I-c(I)\right] \frac{1}{\Delta_{\xi}}<0 .
\end{gathered}
$$

Hence, a planner who cares primarily about $E$ would like to increase $I$ and reduce $\xi^{*}$.

Definition 2. The social planner's equilibrium is defined as the set of bank assets, $\{I, L\}$, bank liabilities, $\{D, E\}$, the run threshold, $\xi^{*}$, deposit rates, $\left\{r_{D}, R_{D}\right\}$, and the loan rate, $R_{I}$, that maximize social welfare, $\mathbb{U}_{S P}^{*}$, defined in (38) subject to the balance sheet (BS) constraint (9), the global game (GG) constraint (32), the deposit supply (DS) schedule (2), and the loan demand (LD) schedule (7).

Each first-order condition in the planner's equilibrium takes the form

$$
\frac{\partial \mathbb{U}_{B}}{\partial \mathcal{C}}+w_{S} \frac{\partial \mathbb{U}_{S}^{*}}{\partial \mathcal{C}}+w_{E} \frac{\partial \mathbb{U}_{E}^{*}}{\partial \mathcal{C}}+\sum_{Y} \zeta_{Y} \frac{\partial \mathcal{Y}}{\partial \mathcal{C}}=0
$$

where $\zeta_{y}$ are the shadow values on the constraints $\mathcal{Y} \in\{B S, G G, D S, L D\}$ (analogous to $\psi_{y}$ in the private equilibrium). The planner's optimality conditions differ from the private ones, because the planner accounts for how her choices affect $S$ and $E$, and thus assigns different shadow values on the same constraints $\mathcal{Y}$. Following the same steps as for the private equilibrium, we can derive the same three margins for the planner (see the Online Appendix for detailed expressions).

The asset allocation margin for the planner can be written as $A A M_{S P}=A A M_{P E}+A A M_{W D}$, where $A A M_{P E}$ is given by (34) and $A A M_{W D}$ is a wedge, which captures the additional distortions that the planner takes into account and will try to correct, given by:

$$
A A M_{W D}=\underbrace{\left(w_{S} \frac{\partial \mathbb{U}_{S}^{*}}{\partial \xi^{*}}+w_{E} \frac{\partial \mathbb{U}_{E}^{*}}{\partial \xi^{*}}\right)\left(\frac{\partial \xi^{*}}{\partial L}-\frac{\partial \xi^{*}}{\partial I}\right)}_{\text {Run consequences from asset allocation }}-\underbrace{w_{E}(1-q) c^{\prime \prime}(I) I}_{\text {Surplus to } E \text { from additional } I} .
$$

The first term in (44) captures the impact on run-risk due to the choice of the asset allocation between liquid assets and loans. If shifting a unit of loans to liquid assets reduces the run probability, i.e., 
$d \xi^{*} / d L-d \xi^{*} / d I$ shown in (C.8) is negative, then because the planner cares about $S$ and $E$, she would want a more liquid asset mix than in the private equilibrium. The last term in (44) captures the surplus created for the entrepreneur from an additional unit of investment, and this consideration leads the planner to increase the level of investment to favor $E$. Note that, in principle, a more liquid asset mix and more investment can be consistent if they are accompanied by a bigger balance sheet.

Similarly, the capital structure margin for the planner is $C_{S M_{S P}}=C S M_{P E}+C S M_{W D}$, where $C S M_{P E}$ is given by (35) and the wedge $C S M_{W D}$ is given by:

$$
C S M_{W D}=-\underbrace{\left(w_{S} \frac{\partial \mathbb{U}_{S}^{*}}{\partial \xi^{*}}+w_{E} \frac{\partial \mathbb{U}_{E}^{*}}{\partial \xi^{*}}\right) \frac{\partial \xi^{*}}{\partial D}}_{\text {Run consequences from liability mix }}+\underbrace{w_{S}\left[U^{\prime \prime}\left(e_{S}-D\right) D+(1-q) V^{\prime \prime}\left(D r_{D}\right) D r_{D}^{2}\right]}_{\text {Surplus to } S \text { from additional } D} .
$$

Shifting funding from deposits to equity reduces run-risk and helps $S$ and $E$ but reduces the surplus to $S$, because the last term is negative (due to concavity of $U$ and $V$ ).

Finally, the deposit loan margin for the planner is $D I M_{S P}=D I M_{P E}+D I M_{W D}$, where DIM $M_{P E}$ is given by (36) and the wedge $D I M_{W D}$ is given by:

$$
\begin{aligned}
D I M_{W D} & =\overbrace{\left(w_{S} \frac{\partial \mathbb{U}_{S}^{*}}{\partial \xi^{*}}+w_{E} \frac{\partial \mathbb{U}_{E}^{*}}{\partial \xi^{*}}\right)\left(\frac{\partial \xi^{*}}{\partial I}+\frac{\partial \xi^{*}}{\partial D}\right)}^{\text {Run consequences of boosting loans and deposits }} \\
& \underbrace{-w_{S}\left[U^{\prime \prime}\left(e_{S}-D\right) D+(1-q) V^{\prime \prime}\left(D r_{D}\right) D r_{D}^{2}\right]+w_{E}(1-q) c^{\prime \prime}(I) I}_{\text {Surplus to } S \text { and } E \text { from boosting loans and deposits }} .
\end{aligned}
$$

If an additional unit of deposits to fund loans increases the probability of a run, i.e., $\partial \xi^{*} / \partial I+$ $\partial \xi^{*} / \partial D>0$, the planner would like to do less of that. Yet, the run considerations may be offset by the second term, because taking more deposits to make more loans increases the surplus to $S$ and $E$.

Having described the various wedges, we have established three of our four main results. First, we have seen that there are three distorted margins that differ between the private and social allocations. Second, all three wedges feature a component linking the structure of the balance sheet to run-risk, and a component that captures the surplus created for either $S$ and/or $E$. The planner trades off reducing run-risk against improving the surplus accruing to $S$ and/or $E$ when the run does not occur. In doing so, she chooses a different asset allocation, capital structure, and level of lending funded by deposits, which have a direct impact on the surplus and an indirect impact on run-risk.

Our third main result is that correcting these distortions involves tradeoffs as it skews allocations toward either favoring $E$ over $S$ or vice versa. For instance, holding more safe assets and fewer loans helps $S$ because it makes their deposits safer. Conversely, holding more loans and fewer safe assets creates more opportunities for $E$ but reduces the buffer that helps mitigate the riskiness of deposits.

In section 4, we show how to implement (decentralize) the planner's solution with regulation. This will take us to our fourth main result that the three distortions are distinct, and three independent tools are generally needed to replicate the planner's allocations.

For completeness, the following Corollary shows the conditions for constrained efficiency. 
Corollary 2. The private and social planner's equilibria coincide if all of the following conditions hold: $\left(\right.$ i) $c^{\prime \prime}()=0$; $\left(\right.$ ii) $V^{\prime \prime}()=0$; and (iii) $U^{\prime \prime}()=0$ or $e_{S}>\bar{e}_{S}$ such that $L_{S}>0$.

Proof. Set $c(x)=a_{c} x$ and $V(x)=a_{v} x$, with $a_{c}, a_{v} \geq 0$ (recall that $\left.c(0)=V(0)=0\right)$. Then, $\partial \mathbb{U}_{E}^{*} / \partial \xi^{*}=$ $-\left[c^{\prime}(I) I-c(I)\right] / \Delta_{\xi}=0$ and $\partial \mathbb{U}_{S}^{*} / \partial \xi^{*}=-\left[V\left(D r_{D}\right)-V^{\prime}\left(D r_{D}\right) D r_{D}\right] / \Delta_{\xi}=0$. Moreover, $(1-q) c^{\prime \prime}(I) I=$ 0 and $(1-q) V^{\prime \prime}\left(D r_{D}\right) D r_{D}^{2}=0$. Given $U^{\prime \prime}=0$, all wedges $A A M_{W D}=C S M_{W D}=D I M_{W D}=0$. Note that $U^{\prime \prime}=0$ occurs endogenously if $L_{s}>0$, since $U^{\prime}=\beta \delta+\beta^{2}(1-\delta)$ from (3).

Corollary 2 says that, if the bank is creating no welfare gains for $S$ and $E$, there is nothing a planner can do to improve outcomes under the assumed market structure. This is not surprising because the private and social allocations differ only because $B$ disregards the direct effects of her choices on $S$ and $E$. If the bank cannot create any value for $S$ and $E$, there is no scope for an intervention by the planner to improve outcomes. In Section 5, we explore extensions of the model under which there are additional reasons why private and social allocations differ. In those cases, a planner might make different choices than private agents even under the conditions in Corollary 2.

Finally, it is also possible that changing the nature of competition in the deposit or loan markets could overturn Corollary 2. For instance, if there were multiple banks competing to raise deposits, it is possible that they would be led to offering contracts that implicitly maximize saver's welfare subject to the banks' participation constraints. In that case, the private choices might match the ones made by the planner when the weights are $w_{S}=1$ and $w_{E}=0$, while banks break-even. A similar argument could be made for the case of severe competition in the loan market. More work investigating the role of competition would be a promising direction for future research.

\subsection{Numerical Example}

This section presents our benchmark numerical example and compares the private equilibrium with the planner's solution under different $w_{E}$ and $w_{S}$, focusing on the distortions in the three margins.

\subsubsection{Parametrization}

The full set of parameters we used to solve the model is shown in Table 1. The parametrization should be taken more as an illustrative example to highlight the mechanisms in the model, rather than as a realistic calibration of the economy that would be suitable for making quantitative statements about the absolute optimal level of banking regulations. We have experimented with various other parameter choices, and the findings that we emphasize are quite robust.

Our model would require further modifications to use it for quantitative policy analysis. For example, all liabilities are unsecured, while, in practice, certain types of deposits are insured. Deposit insurance, even if partial, would reduce the reliance on market discipline exerted by depositors. Hence, deposit rates would be much lower, as we see in advanced economies. It is also not clear whether the various regulations in practice (Basel requirements, stress tests) are binding and whether one should be calibrating to match a regulated economy rather than an unregulated private equilibrium. Another potential concern is that our model consists of three periods, so one would need to 
decide how to interpret the length of each period. Finally, the quasi-linearity of preferences as well as the finite horizon of the model, make depositors willing to accept a higher probability of a run than if they were risk averse or if there was a continuation value for the bank. One could add convex bankruptcy costs to mimic a higher degree of risk-aversion as well as model the continuation value, but doing so is not important to make our fundamental analytic points.

With these caveats in mind, let us call attention to some of the considerations we took into account while choosing the model parameters. Our objective in picking them was to obtain a private equilibrium that is rich enough to describe the mechanisms we want to highlight, rather than to match observable variables. First, the bank is profitable enough, and the initial equity of the banker and her preference for current consumption are such that she voluntarily uses some of her endowment to buy more equity in the bank. So the banker finds operating the bank to be profitable. Second, the deposit services provided by the bank lead savers to forgo self insuring by directly holding the liquid asset. If savers were opting to self insure, then the banking sector is underperforming as a provider of liquidity and, hence, regulations that makes the bank more stable would have an additional positive effect. Third, we have chosen the parameters so that the bank makes loans and invests in liquid assets, but also plans to liquidate some loans to serve early withdrawals. The key parameters responsible for this outcome are the size of the liquidity preference shock, the distribution of the liquidation values for recalled loans, and the riskiness of investment. Fourth, we have chosen $\log$ utility for $t=1$ consumption for both the savers and the banker, while the banker values future consumption more than the savers do. In particular, $U(x)=\log (x)$ and $W(x)=\gamma \log (x)$, where $\gamma<1 .{ }^{14}$ Finally, we set $V(x)=c_{D} \log (1+x)$ and $c(x)=c_{I} x^{\phi_{I}}$, with $\phi_{I}>1$, which satisfy the general properties that were assumed for the transaction services' and effort cost functions.

\subsubsection{Comparing the Private and Social Equilibria}

Tables 2 shows the $P E$ and $S P$ outcomes for small perturbation of the welfare weights away from the private equilibrium. ${ }^{15}$ We focus the analysis around the three margins derived in section 3.1.

Before turning to the details, it is helpful to recall three things about the nature of the distortions that the planner is trying to correct. First, the banker is already internalizing everything that matters for her own welfare. The problem is that she is ignoring the consequences of her choices on the saver and the borrower. Therefore, anything the planner does to take this into account will make the banker worse off. So the planner will be constrained on this front by the need to make sure that the banker will still find it profitable enough to monitor loans. The saver generally wants safer deposits. This can be accomplished by reducing the riskiness of the asset mix or by raising more equity from the banker. The banker will only contribute more equity if the expected profit yield is high enough.

\footnotetext{
${ }^{14}$ Assigning to the banker the same utility function requires high enough $e^{B}$ or low enough $\gamma$ such that she invests enough of her wealth in equity to provide benefits to savers. We do the second because we want the banker endowment to represent only a small part of the total endowment in the economy, with the vast majority accruing to the savers. For $\gamma=1 / \beta^{2}$, such that savers and the banker discount the future the same way, and for log utility, we can obtain the same equilibrium for banker's wealth $\breve{e}^{B}=E+\left(e^{B}-E\right) /\left(\beta^{2} \gamma\right)$, where $E$ is the equilibrium value of contributed equity.

${ }^{15}$ Given $t=1$ allocations, $\xi^{*}$ is unique, but there may be other private equilibria each with a different unique $\xi^{*}$.
} 
Finally, the borrower would like to get more loans, but since the demand curve is downward sloping, more lending requires lower interest rates.

Initially, consider the case where the planner favors $E$ and $S$ equally-for example, $w_{E}=w_{S}=0.1$ in Table 2. To address run-risk, the planner would like to increase liquid holdings in the asset mix, because $\partial \xi^{*} / \partial L-\partial \xi^{*} / \partial I<0$ in (44), and increase the amount of equity in the liabilities mix, because $\partial \xi^{*} / \partial D>0$ in (45). However, to benefit $E$ and $S$, the planner needs to pay attention to the level of loans and deposits such that they do not drop. To achieve these various goals the overall size of the bank's balance sheet should grow, while making assets more liquidity intensive and liabilities more equity intensive. The reduction in run-risk mitigates the upward pressure on the deposit rate, and the volume of lending has to be high enough so that, even though the loan rate will be lower, expected profits grow by enough to induce the banker to supply more equity.

One way to think about what is happening in this experiment is to recognize that, in the private equilibrium, the banker is restricting lending to prop up loan rates and limiting deposits to suppress the cost of deposits. The planning allocations correct these problems. In doing so, the banker is made slightly worse off, but the other two agents are much better off. Overall, social welfare rises.

Next, consider the case that the planner wants to favor $S$, but cares little about $E$; for example $w_{E}=0$ and $w_{S}=0.2$. Similar to the first case, the planner would like to improve the liquidity of the asset mix to address run-risk. But, the planner is now less concerned about the surplus accruing to $E$ and can more easily shift some of the investment towards the liquid asset. At the same time, the planner would like to increase equity in the liabilities mix in order to address the run-risk but without cutting deposits, which is what matters for the surplus accruing to $S$. The planner can increase both liquidity and deposits and, at the same time, guarantee that $B$ will inject more equity in the bank by cutting the level of lending. This set of changes is enough to make equity funding attractive for the banker because the loan-deposit spread rises boosting bank profitability. The overall size of the balance sheet goes up, but the amount of lending that is deposit funded falls.

Finally, consider the case where the planner wants to favor $E$ but cares little about $S$; for example, $w_{E}=0.2$ and $w_{S}=0$. The planner would like to increase lending and the surplus accruing to $E$. Thus, the planner shifts liquidity to loans, which makes deposits more expensive. The planner could then substitute away from deposits to equity to fund the higher investment, but this would only be possible if such a shift is accomplished by maintaining enough profitability so that equity injection by the banker remains attractive. Given the optimality of private allocations from $B$ 's perspective, such a shift from deposits to equity -with a shift from liquidity to loans at the same time- would not be profitable for her. However, because the planner is not concerned with helping $S$, it is possible to reduce deposit-taking, which lowers the deposit rate and also the necessary amount of liquidity that is needed to be carried for early withdrawals. The planner shifts allocations so that more deposits are being used to support lending, even though the bank operates with a smaller balance sheet. 


\subsubsection{Lending, Run-Risk and Welfare in Private and Social Equilibria}

The private equilibrium may exhibit over- or under-investment (or lending) compared with the planner's outcomes depending on $w_{E}$ and $w_{S}$. Lending falls and liquid asset holdings rise with higher $w_{S}$. Both factors contribute to a higher liquidity ratio $\ell \equiv L /(L+I)$. Lower lending is accompanied by a higher loan rate, and this induces the banker to provide more equity. The leverage ratio $k \equiv E /(E+D)$ improves as a result. Even though this also helps deposits increase somewhat, the amount of deposits that support lending is falling, so that the loan-deposit spread rises. The opposite is true when $w_{E}$ is higher. In this case, investment jumps, but deposits fall.

Naturally, the run probability is lower when more weight is placed on $S$. But, lower run-risk accompanied by higher $\ell$ and $k$ can still be consistent with higher lending. This can happen when the bank has a bigger balance sheet and uses more deposit funding to support lending. Notice that this is what happens when the planner cares equally about entrepreneurs and savers.

The enhanced stability of both the asset portfolio and the capital structure of the bank is beneficial to $S$ especially because, as discussed above, it can be accompanied by a higher level of deposit services. Lower run-risk is also beneficial to $E$ but may come at the cost of lower investment and, hence, lower surplus from production. Indeed, when investment falls below its level in the private equilibrium, the entrepreneur will be worse off. As more weight is placed on $E$, the level of investment increases, pushing up the run probability and reducing the surplus from deposit services. $S$ 's welfare goes down, and, after a point, she is worse off compared with the private equilibrium. In this example, both $S$ and $E$ can be made better off when the planner cares about them equally. $B$ is always worse off, because she already internalized what mattered for her and any deviation from the private equilibrium reduces her welfare. Note that the planner not only increases social welfare $\mathbb{U}_{s p}=\mathbb{U}_{B}+w_{S} \mathbb{U}_{S}^{*}+w_{E} \mathbb{U}_{E}^{*}$, which depends on the weights, but also the overall surplus in the economy, $\mathbb{S}_{s p}=\mathbb{U}_{B}+\mathbb{U}_{S}^{*}+\mathbb{U}_{E}^{*}$. Thus, the planner could improve the welfare of all agents if she were able to make lump-sum transfers across agents.

\section{Regulation}

We now explore how the planner's solution can be decentralized via various regulatory interventions. We group tools into two categories. The first category includes tools that target the capital structure in order to get more equity in the bank. The second category includes tools that aim to make the asset mix more liquid. Sections 4.1 and 4.2 discuss the effects when the tools are used in isolation. Section 4.3 discusses how the regulations can be optimally combined.

Recall that the leverage and liquidity ratios are $k \equiv E /(I+L)$ and $\ell \equiv L /(I+L)$. Also, denote the risk-weighted capital ratio by $C R \equiv E / I$ (with zero weight on $L$ and weight of one on $I$ ), the liquidity coverage ratio by $L C R \equiv(L+\xi I) /\left(D r_{D}\right)$, and the net stable funding ratio by $N S F R \equiv$ $E+(1-\delta) D) / I$. On top of these regulatory ratios, consider a tax (subsidy) $\tau_{D}$ on $D$, a tax (subsidy) $\tau_{I}$ on $I$, a tax (subsidy) $\tau_{E}$ on $E$, and a tax (subsidy) $\tau_{L}$ on $L$. 


\subsection{Capital Regulation}

We examine higher requirements for $k$ and $C R$. The direct effect of both tools would be to increase the level of equity in the bank's liabilities. Both $k$ and $C R$ will affect the probability of a run directly by changing allocations in (32), and indirectly by influencing loan and deposit rates. It is helpful to keep in mind that the banker will only invest in additional equity if the bank profits rise. Profitability depends on the spread between the $R_{I}$ and $R_{D}$, so rising profitability requires either higher loan rates or lower deposit rates. Hence, either lending will have to fall so that the loan rate can rise, or deposits have to be made safer so that the deposit rate can fall. We, first, show that the direct effect (i.e., keeping rates and other allocations constant) of higher $k$ or $C R$ is to reduce run-risk.

Proposition 3. For $X$ low enough, the partial equilibrium effect of higher $k$ or $C R$ on $\xi^{*}$ is negative.

Proof. Set $X=0$ to make the determination of $\xi^{*}$ via (32) scale invariant assuming constant lending and deposit rates. Dividing by $I+L,(32)$ can be expressed in terms of $k$ and $\ell$, yielding the following derivative with respect to $k$, keeping $\ell$ and lending/deposit rates constant:

$$
\frac{\partial G G}{\partial k}=\left(\frac{\xi^{*} R_{D}}{R_{I} r_{D}-\xi^{*} R_{D}}+\delta\right) \omega R_{D}+\left(\theta\left(\xi^{*}\right)-\delta\right) r_{D}>0 .
$$

Thus, $\partial \xi^{*} / \partial k=-(\partial G G / \partial k) /\left(\partial G G /\left.\partial \xi\right|_{\xi=\xi^{*}}\right)<0$, since $\partial G G /\left.\partial \xi\right|_{\xi=\xi^{*}}>0$ from Proposition 1. Also, $C R=k(1-\ell)$. So higher $k$ is equivalent to higher $C R$, all else being equal, and, thus, higher $C R$ also reduces $\xi^{*}$. By continuity, the Proposition holds for positive but low enough $X$.

Hence, in partial equilibrium, tightening $k$ or $C R$ is the same as long as $\Delta k=\Delta C R(1-\ell)$, where $\ell$ is the liquidity ratio in the private equilibrium.

However, the general equilibrium effects of the two regulations on the incentive of bankers to hold liquidity and on the deposit supply and loan demand will differ. These differences stem from the way that $k$ and $C R$ affect the three margins. Both affect $C S M$ and DIM in the same way by requiring more equity relative to deposits. Increasing $k$ does not affect the $A A M$ because the bank can costlessly switch from liquid assets to loans. This substitution is only possible if lending rates fall, implying that deposit rates must also fall to boost profitability. By contrast, increasing $C R$ directly affects the $A A M$ by lowering lending, which will lead to a higher loan rate. The higher loan rate means that the bank can take on more deposits than if lending had risen.

Table 3 reports the results for individually tightening $k$ and $C R$, with $\Delta k=\Delta C R(1-\ell)$. We show cases that both requirements increase by a little and by a lot. The beneficial effect on run-risk continues to hold with general equilibrium effects under both regulations. However, the magnitude of the decrease differs and so do the effects on other components of the bank's balance sheet.

First, consider the case where $k$ and $C R$ are marginally tightened. To compare the effects, notice how the $A A M$ is differentially impacted. Loans decrease much more under $C R$ than $k$, which improves bank's asset liquidity. Thus, run-risk goes down even further than what we would expect by just increasing capital. The lower run-risk makes deposits cheaper, and the bank increases its 
deposit-taking. $S$ gains, while both $E$ and $B$ lose. The results are similar for $k$, but the effects are less pronounced because the bank can shift some liquidity to lending and still satisfy the regulation. Because of the additional lending, the bank does not boost liquidity by as much, so the scope to increase deposits is reduced. The gains for $S$ are smaller and so are the losses for $E$.

Considering a much larger increase in capital, we obtain the same but stronger effects for $C R$. However, for $k$, the substitution towards lending becomes much more pronounced. The bank actually increases lending relative to the private equilibrium and substantially reduces its holding of liquid assets. ${ }^{16}$ Given those changes, the cost of deposits must fall in order to make the bank profitable enough to support the higher level of capital. This drop occurs because the total deposits fall, and $S$ are made worse off from this change. The higher level of lending make $E$ better off.

\subsection{Liquidity Regulation}

We examine higher regulatory requirements on $\ell, L C R$, and NSFR.

Proposition 4. For $X$ low enough, and $\delta>\exp ^{-1}$ or $\ell>\hat{\ell} \in R$, the partial equilibrium effect of higher $\ell, L C R$, or NSFR on $\xi^{*}$ is negative.

Proof. Setting $X=0$ and following the same steps as in the proof of Proposition 3, the derivative of $G G$ with respect to $\ell$, keeping $k$ (and lending/deposit rates) constant, is:

$$
\frac{\partial G G}{\partial \ell}=\left(1-\xi^{*}\right)\left[\frac{\omega R_{D} R_{I}}{R_{I} r_{D}-\xi^{*} R_{D}}+\log \theta\left(\xi^{*}\right)\right]
$$

which is positive if $\log \theta\left(\xi^{*}\right)>-1$ given that $\omega R_{D}>r_{D}$ and Lemma 2. In turn, this is satisfied under sufficient conditions $\delta>\exp ^{-1}$ since $\theta\left(\xi^{*}\right)>\delta$ or $\ell>\hat{\ell} \equiv\left[\exp ^{-1}(1-k) r_{D}-\xi^{*}\right] /\left(1-\xi^{*}\right)$, which is true for high enough $\xi^{*}$. Also, $L C R=((1-\underline{\xi}) \ell+\underline{\xi}) /\left(k r_{D}\right)$ and NSFR $=(k+(1-\delta)(1-k)) /(1-\ell)$. So increasing $\ell$ is equivalent to increasing $L C R$ or $N S F R$, all else being equal, and, thus, higher $L C R$ or NSFR reduce $\xi^{*}$. By continuity, the Proposition holds for positive but low enough $X$.

This proposition establishes that, if one fixes the leverage ratio, $k$, as well interest rates, then tightening $\ell, L C R$, or $N S F R$ reduces run-risk in exactly the same way, because $L C R=((1-\xi) \ell+$ $\xi) /\left((1-k) r_{D}\right)$ and $N S F R=(k+(1-\delta)(1-k)) /(1-\ell)$. Hence, in a partial equilibrium setting, one would conclude that these three regulations are equivalent.

These regulations have the unintended consequence that extra liquidity raises patient savers' incentives to join the full-run. We show that these perverse incentives do not dominate when the fraction of patient depositors is small enough or when the liquidity ratio in the private equilibrium is above a cutoff. In the private equilibrium we examine, $\hat{\ell}<0$ because the run-risk is big enough to limit the strength of this channel, so the partial equilibrium effect of higher $\ell$ on $\xi^{*}$ is negative.

As with capital regulations, the general equilibrium effects of liquidity regulations may differ because they alter the three critical margins differently. Table 4 reports the results for individually

\footnotetext{
${ }^{16}$ This is in contrast to models where the bank cannot raise additional equity, where stricter capital/leverage requirements (mechanically) result in a drop in credit extension (see, for example, Clerc et al., 2015; and the references therein).
} 
tightening $\ell, L C R$, or NSFR, with $\Delta L C R=\Delta \ell(1-\underline{\xi}) /\left((1-k) r_{D}\right)$ and $\Delta N S F R=\Delta \ell /(1-\ell) N S F R$, where $k, N S F R$ and $\ell$ are the leverage, net stable funding, and liquidity ratios in $P E$.

First, focus on $\ell$ and $L C R$, which are very similar. Mandating that the bank must hold more liquidity changes the trade-off between investing in risky loans and liquid assets. The higher liquid asset holdings allow the bank to raise more deposits without increasing run-risk. Although deposits increase, the portion that is channeled to loans falls. At the same time, equity goes up.

Why does requiring to hold more liquidity induces the bank to both raise more deposits and more equity? The bank can raise more deposits and invest them in the liquid asset to satisfy the regulation. This is preferable to raising equity in order to invest in the liquid asset, because equity is more expensive. Despite the fact that run-risk decreases, the increased demand for deposits pushes up the deposit rate and, thus, makes loans less profitable. The bank will reduce lending to secure higher loan rates to raise profitability. For a large enough fall in lending, the loan-deposit spread widens so much that it becomes desirable to increase the amount of equity.

The NSFR regulation operates via the same channels, but the effects are less pronounced. The reason is that this regulation also partially resembles a risk-weighted capital requirement. Notice that the $N S F R$ can be rewritten as $1-\delta+\delta C R+(1-\delta) \ell /(1-\ell)$ so that it operates through affecting both capital and liquidity. Relative to the other liquidity regulations, the NSFR has a milder effect on deposits. $S$ is better off, while $E$ and $B$ are worse off under all liquidity regulations.

\subsection{The Optimal Regulatory Mix}

We examine if and how regulation can be combined to implement the planner's solution as a private equilibrium. We consider the following set of tools $\mathbb{T}=\left\{k, C R, \ell, L C R, N S F R, \tau_{D}, \tau_{I}, \tau_{E}, \tau_{L}\right\}$. We expand the set of tools beyond the capital and liquidity regulations for two reasons. First, for managing the loan-deposit spread, these tools are inadequate. Second, as we will now see, it is simpler to describe how the distorted wedges are connected using the tax/subsidy tools. In part, this is because using the liquidity and capital tools requires checking if they are binding or not. ${ }^{17}$

The following Proposition establishes our fourth main result.

Proposition 5. For non-zero and linearly independent wedges (44), (45), and (46), three tools are needed to replicate the planner's preferred allocations.

(i) Any combination of three tools among $\tau_{D}, \tau_{I}, \tau_{E}$ and $\tau_{L}$ can be used to implement the planner's solution.

(ii) If $C R<A A M_{W D} /\left(A A M_{W D}+C S M_{W D}+D I M_{W D}\right)<1-\ell$, and $\delta>\exp ^{-1}$ or $\ell>\hat{\ell}$, the planner's solution can be implemented with a combination of $C R$, $\ell$, and $\tau_{D}$.

(iii) If $C R>A A M_{W D} /\left(A A M_{W D}+C S M_{W D}+D I M_{W D}\right)$, and $\delta>\exp ^{-1}$ or $\ell>\hat{\ell}$, the planner's solution can be implemented with a combination of $C R$, $\tau_{I}$, and $\tau_{D}$.

\footnotetext{
${ }^{17}$ Without loss of generality, the easiest way to model the additional tools is to tax (reward) the banker $\tau_{D}, \tau_{I}, \tau_{E}$ and $\tau_{L}$ per unit of $D, I, E$ and $L$, and then return to (extract from) her a lump-sum amount equal to $\tau_{D} D, \tau_{I} I, \tau_{E} E$ and $\tau_{L} L$.
} 
Proof. To replicate the $S P$ solution as a $P E$, the tools must alter the margins to equate the wedges in (44), (45), and (46); see the Online Appendix for why this is a necessary and sufficient condition. Implementation is simple using taxes/subsidies chosen independently. Given that there are three independent wedges, a combination of three taxes is needed, establishing (i).

The situation is less straightforward with capital and liquidity regulations as the Lagrange multipliers on them need to be positive so that regulations are binding. Take, for example, an implementation with $C R$, $\ell$, and $\tau_{D}$. Implementation requires that $\psi_{C R} C R+\psi_{\ell}=A A M_{W D}, \psi_{C R}+\tau_{D}=C S M_{W D}$, and $-\psi_{C R} C R-\psi_{\ell} \ell-\tau_{D}=D I M_{W D}$, where $\psi_{C R}$ and $\psi_{\ell}$ are the multipliers on capital and liquidity requirements $E \geq I \cdot C R$ and $(1-\ell) L \geq \ell I$. Solving for multipliers and the $\tau_{D}$, we get $\psi_{C R}=\left(A A M_{W D} \ell+C S M_{W D}+D I M_{W D}\right) /(1-C R(1-\ell)), \psi_{\ell}=\left(A A M_{W D}-\left(A A M_{W D}+C S M_{W D}+\right.\right.$ $\left.\left.D I M_{W D}\right) C R\right) /(1-C R(1-\ell))$, and $\tau_{D}=\left(-A A M_{W D} \ell-C S M_{W D} C R(1-\ell)-D I M_{W D}\right) /(1-C R(1-$ $\ell)$ ). Note that $A A M_{W D}+C S M_{W D}+D I M_{W D}=\left(w_{S} \partial \mathbb{U}_{S}^{*} / \partial \xi^{*}+w_{E} \partial \mathbb{U}_{E}^{*} / \partial \xi^{*}\right) \partial \xi^{*} / \partial L>0$ from Proposition 4 for $\delta>\exp ^{-1}$ or $\ell>\hat{\ell}$. Thus, $\psi_{C R}, \psi_{\ell}>0$ if the conditions in (ii) hold.

The implementation with $C R, \tau_{I}$, and $\tau_{D}$ requires $\psi_{C R}=A A M_{W D}+C S M_{W D}+D I M_{W D}>0$, $\tau_{I}=A A M_{W D}-\left(A A M_{W D}+C S M_{W D}+D I M_{W D}\right) C R$, and $\tau_{D}=-\left(A A M_{W D}+D I M_{W D}\right) ; \tau_{I}$ is positive under (ii) and negative under (iii), also verifying that $\ell$ can be part of the optimal mix only in (ii).

Proposition 5 shows that typically capital requirements and deposit-taking subsidies are useful ingredients of the optimal regulatory mix. The third tool with which they would be combined varies and depends on the sign and size of $A A M_{W D}=\left(w_{S} \partial \mathbb{U}_{S}^{*} / \partial \xi^{*}+w_{E} \partial \mathbb{U}_{E}^{*} / \partial \xi^{*}\right)\left(\partial \xi^{*} / \partial L-\partial \xi^{*} / \partial I\right)-$ $w_{E}(1-q) c^{\prime \prime}(I) I$, which depends on the weights $w_{S}$ and $w_{E}$. Setting $w_{E}=0$, the wedge captures the increase in savers' utility from lower run-risk that follows from requiring higher liquid asset holdings. The higher are these holdings, the easier it is to satisfy the condition in part (ii) of Proposition 5. However, for large enough $w_{E}, A A M_{W D}$ may become negative, so that case (iii) of Proposition 5 could obtain, justifying a lending subsidy. This is intuitive because the cost to entrepreneurs from lower lending is more important when $w_{E}$ is higher. In general, combining capital regulation and deposit-taking subsidies benefits savers and entrepreneurs as they enhance banking stability, but the use of tools to restrict or encourage lending depends on which agents are favored most.

Having established the general properties of the optimal regulatory mix, examining it in within the context of our numerical example, can provide additional insights. Table 5 reports the outcomes of combining regulatory tools and compares them to the planner's solution for a case where the planner favors the saver. We combine one capital regulation and one liquidity regulation with a tool that can push the bank towards the socially optimal level of deposit funded loans. As already mentioned, there are several potential candidates for the third type of tool, but we will consider a simple tax (or subsidy) on deposit-taking. This tool can indirectly control the spread between loans and deposits, which, as discussed in Corollary 1, is a sufficient statistic for the DIM (provided that the AAM and CSM have been fixed by a capital and a liquidity tool). ${ }^{18}$

\footnotetext{
${ }^{18}$ The planner cannot use two liquidity or capital tools at the same time because they will not be jointly binding. This result is consistent with the analysis in Checchetti and Kashyap (2016), who show that LCR and NSFR regulations almost surely will never bind at the same time. However, the collinearity of the $C R$ and $k$ regulations may be specific to our model
} 
As discussed, tightening $C R$ to target the $C S M$ increases the equity in the bank, reduces run-risk and results in lower loan extension. If, in addition, we impose a stricter $\ell$, we get closer to the planner in terms of the asset and capital structure margins. But, the loan-deposit rate spread (or the amount of deposits funding investment), moves in the opposite direction of what the planner seeks. A subsidy of $\tau_{D}=-9.17 \%$ can be levied to induce the bank to move the spread to where the planner prefers (this subsidy is equivalent to a subsidy of $\tilde{\tau}_{D}=-7.40 \%$ on deposit interest expenses). The combination of the three tools can implement the planner's solution as a private equilibrium. More generally, using two tools that are not redundant would also typically improve welfare relative to any single regulation. However, to mimic the planner, three regulations are required.

The planner puts more emphasis on reducing run-risk and increasing the surplus from deposit services for this set of weights. Hence, capital and liquidity regulations are useful, because they reduce run-risk without impeding deposit-taking (see sections 4.1 and 4.2). Moreover, in view of Corollary 1 and the fact that the asset allocation and capital structure wedges are independent, both regulations are needed. The complementarity of the capital and liquidity regulations arises in this case because agents' welfare is affected by both the bank's asset and liability mix. If, instead, the regulator had an ad hoc objective to enhance stability by reducing run-risk, then either capital or liquidity regulation could be used to accomplish this, and the tools would be substitutes.

Finally, one might expect that capital and liquidity requirements are not useful when the planner would like to increase the lending in the economy (as would be the case when the planner puts high weights on $E$ ). Table 6 shows partial and full implementation of the planner's solution for case. To mimic the planner's outcome, we need to use deposit and lending subsidies. The complication arises because, as we see from Table 2, the planner wants higher lending but much less liquidity for this set of weights. So raising $\ell$ will mean that the planner's allocations cannot be achieved. A $C R$ can be helpful, but it will have to be combined with lending or deposit subsidies. The second column in the table shows what happens when a capital requirement, $C R=4.25 \%$, and a deposit subsidy, $\tau_{D}=-2.20 \%$, are combined, while the third column shows that two subsidies, $\tau_{D}-2.20 \%$ and $\tau_{I}=-4.33 \%$, together can boost lending all the way to where the planner prefers.

Hence, we consider how a lending subsidy can be part of regulatory mix. The last column in the table shows that a lending subsidy, $\tau_{I}=-4.33 \%$, can be combined with $C R=4.25 \%$ and $\tau_{D}=-2.20 \%$ to replicate the planner's allocation (column 5). In this case, capital regulation is needed because the bank is tempted to maximize its leverage to reap the benefits of the subsidies. This helps a bit with respect to run-risk compared with the case that only the two subsidies are in place (column 4), but, unlike a liquidity regulation, capital regulation does not restrict lending materially, which is the primary objective of the planner for this set of weights.

Our findings suggest that capital is useful as part of the optimal regulatory mix irrespective of which agent the planner favors, while liquidity requirements are more useful for savers.

and may not even hold for high $k$ (see the discussion in section 4.1). 


\section{Extensions}

To check the generality of our results, we extend the model in several dimensions. Our main results continue to hold. The details are presented in different subsections of the Online Appendix.

Section D.1 considers the case when the banker does not internalize the effect of her actions on $\xi^{*}$, resulting in higher risk in the private equilbrium. Pareto improvements are possible in this case. Section D. 2 considers incomplete deposit contracts and a lack of commitment by the banker such that the banker has an incentive to deviate towards lending more, holding fewer liquid assets, and raising less equity after the deposit contract has been signed. Again, we show that the need for regulation is stronger and Pareto improvements are possible. Section section D. 3 considers a banker that takes the loan rate $R_{I}$ as given. Lending is higher in the private equilibrium, while the need for regulation, especially liquidity regulation, remains. Section D.4 considers a perfectly elastic loan demand curve such that $E$ drops out of the problem. Results are consistent with those described earlier when the planner puts higher weight on $S$. Section D.5 derives the equilibrium under direct lending from $S$ to $E$ and shows that $S$ would prefer to use a bank due to the payment services and the more efficient monitoring it provides. Section D.6 allows for negative deposit rates for early withdrawals. Our results continue to hold when run-risk is present in equilibrium. We also consider negative deposit rates that implement the run-proof equilibrium and show that it is dominated by the one with positive run-risk that we study. Section D.7 examines when banks can also raise outside equity, which does not affect our results, while section D.8 considers a planner that can use tools to distort the deposit supply and loan demand schedules of savers and entrepreneurs.

\section{Conclusions}

Banks perform important services using both sides of their balance sheet. Yet, private choices made by banks may not be socially optimal and regulating banks' activities can improve social welfare. We examined how many of the regulations that are often mentioned in policy discussions perform in a relatively familiar model of banks. We started from the DD benchmark precisely because it is so thoroughly studied. The modifications we study balance tractability to keep the model relatively simple, against our preference for additional realistic forces that the baseline model excludes.

Our modifications generate endogenous credit risk in banks' portfolios as well as the risk of an endogenous funding run. This simple pair of features interact in interesting and unexpected ways. We draw several lessons from the model that we believe will carry over to many other models.

First, we identify three general margins that are distorted: the relative amounts of liquid and illiquid assets, the mix of deposits and equity, and the spread between loan and deposit rates. Second, the way that a bank privately sets these margins diverges from what a social planner would choose, because of the failure to fully account for the risks created by runs.

Third, the way the planner corrects the distortions in the private equilibrium depends on the relative importance placed on savers versus borrowers. If the social planner cares sufficiently about savers, the planner chooses relatively more liquidity and equity than the banker and would reduce 
bank profits by boosting deposit rates and lowering loan rates. Thus, the planner reduces run-risk, improves the provision of liquidity, guarantees a more stable extension of credit and real production, and delivers more loans funded by deposits compared with the private equilibrium. Capital regulation is still desirable even when the planner cares little about savers, but liquidity regulation needs to be replaced with tools that encourage credit extension and, hence, actually create more risk.

Fourth, more than one regulatory tool is needed to implement the socially optimal allocations. Optimal policy in models without all of these distortions can be misleading. For example, if the liability structure is constrained, say because deposit levels are exogenously determined and equity is fixed, studying asset allocations and distortions becomes much easier. But, regulation, if any is needed, will amount to fixing liquidity ratios. Similarly, shutting down the liquidity demand and liquidity risk makes it easier to focus on the optimal capital structure and level of investment. But, regulation, if again any is needed, would amount to fixing capital ratios. Instead, when both sides of the bank's balance sheet are endogenously determined the distortions from each side interact and a combination of both capital and liquidity requirements emerge in the optimal regulatory mix.

Moreover, our analysis highlights some political economy aspects of regulation that deserve attention. The banker internalizes how her decisions matter for run-risk, funding structure, and the level of lending to maximize her own welfare. Her choices, from a social point of view, have real macroeconomic consequences. Regulation improves aggregate welfare but reduces the rents accruing to bankers. Therefore, incentives to engage in regulatory arbitrage could be strong. The lack of regulatory arbitrage in the model we have studied is one of its main shortcomings.

There are other interesting avenues to extend our model, some of which we have already mentioned and are analyzed in the online appendix. One further direction would be to allow the issuance of long-term debt together with demandable deposits and equity. Including loss-absorbing debt instruments in the regulatory mix could introduce additional ways to tackle with run-risk and credit risk. But it would not constitute a full remedy by itself due to the disciplinary role that demandable liabilities play. Moreover, our model is flexible enough to incorporate fire-sale dynamics by endogenizing the liquidation value of long-term investment. Although this would introduce pecuniary externalities as an additional reason why private allocations are inefficient, it would not qualitatively overturn our main conclusions. Finally, one could enrich the set of risky investments from which a banker could choose and, thus, increase the scope for asset substitution. Setting the (relative) risk-weights in capital requirements to capture social risks would then be highly important.

\section{References}

Allen, F., Carletti, E., Goldstein, I. and Leonello, A. (2018), 'Government guarantees and financial stability', Journal of Economic Theory 177, 518-557.

Allen, F. and Gale, D. (1998), 'Optimal financial crises', Journal of Finance 53(4), 1245-1284.

Allen, F. and Gale, D. (2004), 'Financial intermediaries and markets', Econometrica 72(4), 10231061. 
Calomiris, C. W. and Kahn, C. M. (1991), 'The role of demandable debt in structuring optimal banking arrangements', American Economic Review 81(3), 497-513.

Carlsson, H. and van Damme, E. (1993), 'Global games and equilibrium selection', Econometrica 61(5), 989-1018.

Chari, V. and Jagannathan, R. (1988), 'Banking panics, information, and rational expectations equilibrium', Journal of Finance 43(3), 749-761.

Checchetti, S. G. and Kashyap, A. K. (2016), 'What binds? Interactions between bank capital and liquidity regulations', in The Changing Fortunes of Central Banking, edited by Philipp Hartmann, Haizhou Huang, Dirk Schoenmaker .

Clerc, L., Derviz, A., Mendicino, C., Moyen, S., Nikolov, K., Stracca, L., Suarez, J. and Vardoulakis, A. P. (2015), 'Capital regulation in a macroeconomic model with three layers of default', International Journal of Central Banking 11(3), 9-63.

Cooper, R. and Ross, T. W. (1998), 'Bank runs: Liquidity costs and investment distortions', Journal of Monetary Economics 41(1), 27-38.

Cooper, R. and Ross, T. W. (2002), 'Bank runs: Deposit insurance and capital requirements', International Economic Review 43(1), 55-72.

Davila, E. and Goldstein, I. (2022), 'Optimal deposit insurance', Journal of Political Economy, forthcoming .

Diamond, D. W. (1984), 'Financial intermediation and delegated monitoring', Review of Economic Studies 51(3), 393-414.

Diamond, D. W. and Dybvig, P. H. (1983), 'Bank runs, deposit insurance, and liquidity', Journal of Political Economy 91(3), 401-419.

Diamond, D. W. and Kashyap, A. K. (2016), 'Liquidity requirements, liquidity choice and financial stability', NBER Working Papers 22053.

Diamond, D. W. and Rajan, R. G. (2000), 'A theory of bank capital', Journal of Finance 55(6), 2431-2465.

Ennis, H. M. and Keister, T. (2005), 'Optimal fiscal policy under multiple equilibria', Journal of Monetary Economics 52(8), 1359-1377.

Ennis, H. M. and Keister, T. (2009), 'Bank runs and institutions: The perils of intervention', American Economic Review 99(4), 1588-1607.

Goldstein, I. and Pauzner, A. (2005), 'Demand-deposit contracts and the probability of bank runs', Journal of Finance 60(3), 1293-1327.

Holmström, B. and Tirole, J. (1997), 'Financial intermediation, loanable funds, and the real sector', The Quarterly Journal of Economics 112(3), 663-691.

Jacklin, C. J. and Bhattacharya, S. (1988), 'Distinguishing panics and information-based bank runs: Welfare and policy implications', Journal of Political Economy 96(3), 568-592. 
Kara, G. I. and Ozsoy, S. M. (2019), 'Bank Regulation under Fire Sale Externalities', The Review of Financial Studies 33(6), 2554-2584.

Keister, T. (2015), 'Bailouts and financial fragility’, Review of Economic Studies 83(2), 704-736.

Morris, S. and Shin, H. S. (1998), 'Unique equilibrium in a model of self-fulfilling currency attacks', American Economic Review 88(3), 587-597.

Morris, S. and Shin, H. S. (2003), 'Global Games: Theory and Applications', In Advances in Economics and Econometrics: Theory and Applications, Eight World Congress, Vol. 1, ed. Mathias Dewatripont, Lars P. Hanse, and Stephen J. Turnovsky. Cambridge: Cambridge University Press.

Peck, J. and Shell, K. (2003), 'Equilibrium bank runs’, Journal of Political Economy 111(1), 103123.

Peck, J. and Shell, K. (2010), 'Could making banks hold only liquid assets induce bank runs?', Journal of Monetary Economics 57(4), 420-427.

Rochet, J.-C. and Vives, X. (2004), 'Coordination failures and the lender of last resort: Was bagehot right after all?', Journal of the European Economic Association 2(6), 1116-1147.

Schilling, L. (2022), 'Optimal forbearance of bank resolution', MPRA Paper No. 112409.

Shleifer, A. and Vishny, R. W. (1992), 'Liquidation values and debt capacity: A market equilibrium approach', Journal of Finance 47, 1343-1366.

Uhlig, H. (2010), 'A model of a systemic bank run', Journal of Monetary Economics 57(1), 78-96.

Van den Heuvel, S. (2022), 'The welfare effects of bank liquidity and capital requirements', FEDS 2022-072 .

Vives, X. (2014), 'Strategic complementarity, fragility and regulation', Review of Financial Studies 27(12), 3547-3592.

Walther, A. (2016), 'Jointly optimal regulation of bank capital and liquidity', Journal of Money, Credit and Banking 48(2-3), 415-448. 
Tables and Figures

\begin{tabular}{lllll}
\hline \hline$e_{S}=2.50$ & $e_{B}=0.10$ & $A=4.50$ & $\omega=80 \%$ & $c_{D}=0.27$ \\
$c_{I}=0.1$ & $\phi_{I}=3$ & $X=0.10$ & $\gamma=0.15$ & $\delta=0.50$ \\
$\beta=0.70$ & $\bar{\xi}=2.20$ & $\underline{\xi}=0.01$ & & \\
\hline \hline
\end{tabular}

Table 1: Parametrization.

\begin{tabular}{lcrrrrr}
\hline \hline & PE & \multicolumn{5}{c}{ SP for weights $\left(w_{E}, w_{S}\right)$} \\
$I$ & & $(0.00,0.20)$ & $(0.05,0.15)$ & $(0.10,0.10)$ & $(0.15,0.05)$ & $(0.20,0.00)$ \\
\cline { 2 - 6 }$L$ & 0.862 & 0.785 & 0.841 & 0.873 & 0.899 & 0.906 \\
$D$ & 0.052 & 0.221 & 0.119 & 0.060 & 0.012 & 0.000 \\
$E$ & 0.875 & 0.962 & 0.919 & 0.894 & 0.873 & 0.867 \\
$R_{I}$ & 0.038 & 0.044 & 0.041 & 0.039 & 0.038 & 0.038 \\
$R_{D}$ & 4.097 & 4.198 & 4.131 & 4.089 & 4.051 & 4.042 \\
$q$ & 1.717 & 1.804 & 1.778 & 1.767 & 1.761 & 1.758 \\
$\ell$ & 0.407 & 0.386 & 0.398 & 0.403 & 0.407 & 0.408 \\
$k$ & 0.057 & 0.219 & 0.124 & 0.065 & 0.013 & 0.000 \\
$R_{I}-(1-\delta) R_{D}$ & 3.239 & 3.296 & 3.242 & 3.205 & 3.170 & 3.163 \\
$I+L$ & 0.914 & 1.006 & 0.960 & 0.933 & 0.911 & 0.906 \\
$I-E$ & 0.824 & 0.741 & 0.800 & 0.834 & 0.861 & 0.867 \\
$E(D i v)$ & 0.745 & 0.755 & 0.750 & 0.747 & 0.743 & 0.743 \\
$\Delta \mathbb{U}_{E}$ & - & $-1.66 \%$ & $-0.44 \%$ & $0.33 \%$ & $1.02 \%$ & $1.19 \%$ \\
$\Delta \mathbb{U}_{S}$ & - & $3.63 \%$ & $1.74 \%$ & $0.71 \%$ & $-0.10 \%$ & $-0.30 \%$ \\
$\Delta \mathbb{U}_{B}$ & - & $-0.44 \%$ & $-0.13 \%$ & $-0.05 \%$ & $-0.08 \%$ & $-0.09 \%$ \\
$\Delta \mathbb{U}_{s p}$ & - & $0.29 \%$ & $0.11 \%$ & $0.05 \%$ & $0.07 \%$ & $0.14 \%$ \\
$\Delta \mathbb{S}_{s p}$ & - & $1.53 \%$ & $1.18 \%$ & $0.99 \%$ & $0.84 \%$ & $0.79 \%$ \\
\hline \hline
\end{tabular}

Table 2: Privately versus Socially optimal solutions. The welfare changes are computed over the level of welfare in the private equilibrium, which is normalized to one for each agent. 


\begin{tabular}{|c|c|c|c|c|c|}
\hline & \multirow[b]{2}{*}{$\mathrm{PE}$} & \multicolumn{2}{|c|}{ Milder increase in } & \multicolumn{2}{|c|}{ Bigger increase in } \\
\hline & & $C R$ & $k$ & $\overline{C R}$ & $k$ \\
\hline$I$ & 0.862 & 0.856 & 0.862 & 0.770 & 0.880 \\
\hline$L$ & 0.052 & 0.064 & 0.055 & 0.224 & 0.019 \\
\hline$D$ & 0.875 & 0.880 & 0.876 & 0.940 & 0.841 \\
\hline$E$ & 0.038 & 0.040 & 0.040 & 0.053 & 0.058 \\
\hline$R_{I}$ & 4.097 & 4.106 & 4.099 & 4.213 & 4.077 \\
\hline$R_{D}$ & 1.717 & 1.713 & 1.711 & 1.703 & 1.605 \\
\hline$q$ & 0.407 & 0.405 & 0.406 & 0.387 & 0.405 \\
\hline$\ell$ & 0.057 & 0.069 & 0.060 & 0.225 & 0.021 \\
\hline$k$ & 0.042 & 0.044 & 0.044 & 0.053 & 0.065 \\
\hline$C R$ & 0.045 & 0.047 & 0.047 & 0.069 & 0.066 \\
\hline$R_{I}-(1-\delta) R_{D}$ & 3.239 & 3.249 & 3.243 & 3.362 & 3.274 \\
\hline$I+L$ & 0.914 & 0.920 & 0.916 & 0.993 & 0.899 \\
\hline$I-E$ & 0.824 & 0.816 & 0.821 & 0.717 & 0.821 \\
\hline$E(D i v)$ & 0.745 & 0.749 & 0.749 & 0.779 & 0.791 \\
\hline$\Delta \mathbb{U}_{E}$ & - & $-0.13 \%$ & $-0.01 \%$ & $-2.01 \%$ & $0.51 \%$ \\
\hline$\Delta \mathbb{U}_{S}$ & - & $0.17 \%$ & $0.02 \%$ & $2.68 \%$ & $-1.23 \%$ \\
\hline$\Delta \mathbb{U}_{B}$ & - & $-0.01 \%$ & $-0.01 \%$ & $-0.61 \%$ & $-1.24 \%$ \\
\hline$\Delta \mathbb{S}_{s p}$ & - & $0.03 \%$ & $0.01 \%$ & $0.05 \%$ & $-1.96 \%$ \\
\hline
\end{tabular}

Table 3: Single capital regulations. The welfare changes are computed over the level of welfare in the private equilibrium, which is normalized to one for each agent.

\begin{tabular}{lcrrr}
\hline \hline & \multicolumn{1}{c}{$\mathrm{PE}$} & $\ell$ & \multicolumn{1}{c}{$L C R$} & NSFR \\
\cline { 2 - 5 }$I$ & 0.862 & 0.746 & 0.747 & 0.770 \\
$L$ & 0.052 & 0.258 & 0.257 & 0.217 \\
$D$ & 0.875 & 0.959 & 0.959 & 0.942 \\
$E$ & 0.038 & 0.045 & 0.045 & 0.044 \\
$R_{I}$ & 4.097 & 4.236 & 4.236 & 4.211 \\
$R_{D}$ & 1.717 & 1.746 & 1.745 & 1.736 \\
$q$ & 0.407 & 0.385 & 0.385 & 0.390 \\
$\ell$ & 0.057 & 0.257 & 0.256 & 0.220 \\
$k$ & 0.042 & 0.045 & 0.045 & 0.045 \\
$L C R$ & 0.069 & 0.277 & 0.276 & 0.238 \\
$N S F R$ & 0.552 & 0.703 & 0.702 & 0.670 \\
$R_{I}-(1-\delta) R_{D}$ & 3.239 & 3.363 & 3.363 & 3.343 \\
$I+L$ & 0.914 & 1.004 & 1.004 & 0.987 \\
$I-E$ & 0.824 & 0.701 & 0.701 & 0.726 \\
$E(D i v)$ & 0.745 & 0.756 & 0.757 & 0.756 \\
$\Delta \mathbb{U}_{E}$ & - & $-2.50 \%$ & $-2.49 \%$ & $-2.04 \%$ \\
$\Delta \mathbb{U}_{S}$ & - & $3.50 \%$ & $3.48 \%$ & $2.75 \%$ \\
$\Delta \mathbb{U}_{B}$ & - & $-0.51 \%$ & $-0.51 \%$ & $-0.35 \%$ \\
$\Delta \mathbb{S}_{s p}$ & - & $0.49 \%$ & $0.49 \%$ & $0.36 \%$ \\
\hline \hline
\end{tabular}

Table 4: Single liquidity regulations. The welfare changes are computed over the level of welfare in the private equilibrium, which is normalized to one for each agent. 


\begin{tabular}{lcrrr}
\hline \hline & \multicolumn{1}{c}{$\mathrm{PE}$} & \multicolumn{1}{c}{$C R$} & \multicolumn{1}{c}{$C R \ell$} & $C R, \ell \& \tau_{D}$ \\
\cline { 2 - 5 }$I$ & 0.862 & 0.829 & 0.776 & 0.785 \\
$L$ & 0.052 & 0.116 & 0.206 & 0.221 \\
$D$ & 0.875 & 0.899 & 0.938 & 0.962 \\
$E$ & 0.038 & 0.047 & 0.044 & 0.044 \\
$R_{I}$ & 4.097 & 4.143 & 4.204 & 4.198 \\
$R_{D}$ & 1.717 & 1.700 & 1.735 & 1.804 \\
$q$ & 0.407 & 0.399 & 0.392 & 0.386 \\
$q f$ & 0.200 & 0.179 & 0.150 & 0.147 \\
$\ell$ & 0.057 & 0.123 & 0.210 & 0.219 \\
$k$ & 0.042 & 0.049 & 0.044 & 0.044 \\
$R_{I}-(1-\delta) R_{D}$ & 3.239 & 3.292 & 3.337 & 3.296 \\
$I+L$ & 0.914 & 0.946 & 0.982 & 1.006 \\
$I-E$ & 0.824 & 0.783 & 0.732 & 0.741 \\
$E(D i v)$ & 0.745 & 0.764 & 0.754 & 0.755 \\
$\Delta \mathbb{U}_{E}$ & - & $-0.75 \%$ & $-1.93 \%$ & $-1.66 \%$ \\
$\Delta \mathbb{U}_{S}$ & - & $0.94 \%$ & $2.57 \%$ & $3.63 \%$ \\
$\Delta \mathbb{U}_{B}$ & - & $-0.15 \%$ & $-0.31 \%$ & $-0.44 \%$ \\
$\Delta \mathbb{S}_{s p}$ & - & $0.04 \%$ & $0.33 \%$ & $1.53 \%$ \\
\hline \hline
\end{tabular}

Table 5: Implementation of the planner's solution for $w_{E}=0.00$ and $w_{S}=0.20$. The welfare changes are computed over the level of welfare in the private equilibrium, which is normalized to one for each agent.

\begin{tabular}{lcrrr}
\hline \hline & \multicolumn{1}{c}{$\mathrm{PE}$} & $C R \& \tau_{D}$ & $\tau_{D} \& \tau_{I}$ & $C R, \tau_{D} \& \tau_{I}$ \\
\cline { 2 - 5 }$I$ & 0.862 & 0.859 & 0.900 & 0.899 \\
$L$ & 0.052 & 0.064 & 0.010 & 0.012 \\
$D$ & 0.875 & 0.886 & 0.872 & 0.873 \\
$E$ & 0.038 & 0.037 & 0.038 & 0.038 \\
$R_{I}$ & 4.097 & 4.103 & 4.050 & 4.051 \\
$R_{D}$ & 1.717 & 1.742 & 1.762 & 1.761 \\
$q$ & 0.407 & 0.406 & 0.408 & 0.407 \\
$q_{f}$ & 0.200 & 0.197 & 0.212 & 0.211 \\
$\ell$ & 0.057 & 0.069 & 0.011 & 0.013 \\
$k$ & 0.042 & 0.040 & 0.042 & 0.042 \\
$R_{I}-(1-\delta) R_{D}$ & 3.239 & 3.231 & 3.169 & 3.170 \\
$I+L$ & 0.914 & 0.923 & 0.910 & 0.911 \\
$I-E$ & 0.824 & 0.822 & 0.862 & 0.861 \\
$E($ Div $)$ & 0.745 & 0.740 & 0.743 & 0.743 \\
$\Delta \mathbb{U}_{E}$ & - & $-0.07 \%$ & $1.03 \%$ & $1.02 \%$ \\
$\Delta \mathbb{U}_{S}$ & - & $0.41 \%$ & $-0.12 \%$ & $-0.10 \%$ \\
$\Delta \mathbb{U}_{B}$ & - & $-0.02 \%$ & $-0.08 \%$ & $-0.08 \%$ \\
$\Delta \mathbb{S}_{s p}$ & - & $0.32 \%$ & $0.84 \%$ & $0.84 \%$ \\
\hline \hline
\end{tabular}

Table 6: Implementation of the planner's solution for $w_{E}=0.15$ and $w_{S}=0.05$. The welfare changes are computed over the level of welfare in the private equilibrium, which is normalized to one for each agent. 


\title{
Optimal Bank Regulation In the Presence of Credit and Run-Risk
}

\author{
Anil K Kashyap \\ Dimitrios P. Tsomocos \\ Alexandros P. Vardoulakis
}

\section{Online Appendix}

Appendix A presents the general proof of uniqueness of the threshold equilibrium.

Appendix B derives the problem of a planner that has access to a certain set of tools.

Appendix C reports several detailed derivations of expressions in the paper. Appendices C.1 and C.2 reports the detailed expressions for the margins in the private and social planner's equilibria, while appendix C.3 derives the partial derivatives reported in the optimality conditions in the paper.

Appendix D reports results for several extensions of the model.

\section{A General proof of uniqueness of threshold equilibrium}

In the paper we focused on the case of vanishing noise $\varepsilon \rightarrow 0$. Here we show that the arguments for existence and uniqueness of a threshold equilibrium generalize for non-vanishing, yet small enough, noise $\varepsilon>0$. Re-stating (27), after setting for notational convenience $\tilde{\xi}_{\theta}(\tilde{x}+\Delta x)=\xi_{\theta}(\tilde{x}+\Delta x)-\Delta x$ and $\tilde{\xi}_{\hat{\lambda}}(\tilde{x}+\Delta x)=\xi_{\hat{\lambda}}(\tilde{x}+\Delta x)-\Delta x$, we get

$$
\bar{\Delta}(\tilde{x}+\Delta x)=-\int_{\tilde{x}-\varepsilon}^{\tilde{\xi}_{\theta}(\tilde{x}+\Delta x)} \frac{(\tilde{\xi}+\Delta x) I+L}{\lambda(\tilde{\xi}, \tilde{x})} \frac{d \tilde{\xi}}{2 \varepsilon}-\int_{\tilde{\xi}_{\theta}(\tilde{x}+\Delta x)}^{\tilde{\xi}_{\hat{\lambda}}(\tilde{x}+\Delta x)} D r_{D} \frac{d \tilde{\xi}}{2 \varepsilon}+\int_{\tilde{\xi}_{\hat{\lambda}}(\tilde{x}+\Delta x)}^{\tilde{x}+\varepsilon}\left(\omega D R_{D}-D r_{D}\right) \frac{d \tilde{\xi}}{2 \varepsilon} .
$$

The thresholds $\tilde{\xi}_{\theta}(\tilde{x}+\Delta x)$ and $\tilde{\xi}_{\hat{\lambda}}(\tilde{x}+\Delta x)$ are obtained by equating $\lambda(\xi, \tilde{x}+\Delta x)$, from (21), to $\theta(\xi)$ and $\hat{\lambda}(\xi)$, from (10) and (19). This is equivalent to equating $\lambda(\tilde{\xi}+\Delta x, \tilde{x}+\Delta x)=\lambda(\tilde{\xi}, \tilde{x})$ to $\theta(\tilde{\xi}+\Delta x)$ and $\hat{\lambda}(\tilde{\xi}+\Delta x)$, respectively, since we have set $\xi=\tilde{\xi}+\Delta x$. That is, $\tilde{\xi}_{\theta}(\tilde{x}+\Delta x)$ and $\tilde{\xi}_{\hat{\lambda}}(\tilde{x}+\Delta x)$ are, respectively, the solutions to

$$
\lambda\left(\tilde{\xi}_{\theta}(\tilde{x}+\Delta x), \tilde{x}\right)=\theta\left(\tilde{\xi}_{\theta}(\tilde{x}+\Delta x)+\Delta x\right)
$$

and

$$
\lambda\left(\tilde{\xi}_{\hat{\lambda}}(\tilde{x}+\Delta x), \tilde{x}\right)=\hat{\lambda}\left(\tilde{\xi}_{\hat{\lambda}}(\tilde{x}+\Delta x)+\Delta x\right) .
$$

The views expressed in this paper are those of the authors and do not necessarily represent those of Federal Reserve Board of Governors, anyone in the Federal Reserve System, the Bank of England Financial Policy Committee, or any of the institutions with which we are affiliated. 
First, we re-iterate the argument for existence. For $\tilde{x}+\Delta x \geq \xi_{\text {ud }}+\varepsilon$, we have $\tilde{\xi}+\Delta x \geq \xi_{\text {ud }}$, and $\tilde{\xi}_{\hat{\lambda}}(\tilde{x}+\Delta x), \tilde{\xi}_{\theta}(\tilde{x}+\Delta x) \leq \tilde{x}-\varepsilon$. Hence, $\bar{\Delta}(\tilde{x}+\Delta x)>0$. For $\tilde{x}+\Delta x<\xi_{l d}-\varepsilon$, we have $\tilde{\xi}+\Delta x<\xi_{l d}$, and $\tilde{\xi}_{\hat{\lambda}}(\tilde{x}+\Delta x)>\tilde{x}+\varepsilon$. Hence, $\bar{\Delta}(\tilde{x}+\Delta x)<0$. All the integrands and the limits of integration in (A.1) are bounded and continuous in $\Delta x$, i.e., $\bar{\Delta}(\tilde{x}+\Delta x)$ is continuous in $\Delta x$. Then, because it is positive and negative for extreme values, a threshold $\Delta x^{*}$, satisfying $\bar{\Delta}\left(\tilde{x}+\Delta x^{*}\right)=0$, exists.

To show uniqueness, take the derivative of $\bar{\Delta}(\tilde{x}+\Delta x)$ with respect to $\Delta x:^{1}$

$$
\frac{d \bar{\Delta}(\tilde{x}+\Delta x)}{d \Delta x}=-\int_{\tilde{x}-\varepsilon}^{\tilde{\xi}_{\theta}(\tilde{x}+\Delta x)} \frac{I}{\lambda(\tilde{\xi}, \tilde{x})} \frac{d \tilde{\xi}}{2 \varepsilon}-\frac{1}{2 \varepsilon} \frac{d \tilde{\xi}_{\hat{\lambda}}(\tilde{x}+\Delta x)}{d \Delta x} \omega D R_{D}
$$

which we will evaluate at the crossing points given by $\bar{\Delta}\left(\tilde{x}+\Delta x^{*}\right)=0$.

Adding and subtracting $1 /(2 \varepsilon) \int_{\tilde{x}-\varepsilon}^{\tilde{\xi}_{\theta}(\tilde{x}+\Delta x)} \tilde{\xi}_{\hat{\lambda}}(\tilde{x}+\Delta x) I / \lambda(\tilde{\xi}, \tilde{x}) d \tilde{\xi}$ to $\bar{\Delta}\left(\tilde{x}+\Delta x^{*}\right)=0$ we get that:

$$
\begin{aligned}
& -\frac{1}{2 \varepsilon} \int_{\tilde{x}-\varepsilon}^{\tilde{\xi}_{\theta}\left(\tilde{x}+\Delta x^{*}\right)} \frac{I}{\lambda(\tilde{\xi}, \tilde{x})} d \tilde{\xi} \\
& =\frac{1}{\tilde{\xi}_{\hat{\lambda}}\left(\tilde{x}+\Delta x^{*}\right)+\Delta x^{*}} \frac{1}{2 \varepsilon}\left[\int_{\tilde{x}-\varepsilon}^{\tilde{\xi}_{\theta}\left(\tilde{x}+\Delta x^{*}\right)} \frac{\left(\tilde{\xi}-\tilde{\xi}_{\hat{\lambda}}\left(\tilde{x}+\Delta x^{*}\right)\right) I+L}{\lambda(\tilde{\xi}, \tilde{x})} d \tilde{\xi}+\int_{\tilde{\xi}_{\theta}\left(\tilde{x}+\Delta x^{*}\right)}^{\tilde{\xi}_{\tilde{\lambda}}\left(\tilde{x}+\Delta x^{*}\right)} D r_{D} d \tilde{\xi}\right] \\
& -\frac{1}{\tilde{\xi}_{\hat{\lambda}}\left(\tilde{x}+\Delta x^{*}\right)+\Delta x^{*}} \frac{1}{2 \varepsilon} \int_{\tilde{\xi}_{\hat{\lambda}}\left(\tilde{x}+\Delta x^{*}\right)}^{\tilde{x}+\varepsilon}\left\{\omega D R_{D}-D r_{D}\right\} d \tilde{\xi} .
\end{aligned}
$$

Substituting (A.5) in (A.4), evaluated at $\Delta x=\Delta x^{*}$, we get:

$$
\begin{aligned}
& \left.\frac{d \bar{\Delta}(\tilde{x}+\Delta x)}{d \Delta x}\right|_{\Delta x=\Delta x^{*}}= \\
& -\frac{1}{\tilde{\xi}_{\hat{\lambda}}\left(\tilde{x}+\Delta x^{*}\right)+\Delta x^{*}} \frac{1}{2 \varepsilon} \omega D R_{D}\left[\left.\frac{d \tilde{\xi}_{\hat{\lambda}}(\tilde{x}+\Delta x)}{d \Delta x}\right|_{\Delta x=\Delta x^{*}}\left(\tilde{\xi}_{\hat{\lambda}}\left(\tilde{x}+\Delta x^{*}\right)+\Delta x^{*}\right)+\left(\tilde{x}+\varepsilon-\tilde{\xi}_{\hat{\lambda}}\left(\tilde{x}+\Delta x^{*}\right)\right)\right] \\
& +\frac{1}{\tilde{\xi}_{\hat{\lambda}}\left(\tilde{x}+\Delta x^{*}\right)+\Delta x^{*}} \frac{1}{2 \varepsilon}\left[\int_{\tilde{x}-\varepsilon}^{\tilde{\xi}_{\theta}\left(\tilde{x}+\Delta x^{*}\right)} \frac{\left(\tilde{\xi}-\tilde{\xi}_{\hat{\lambda}}\left(\tilde{x}+\Delta x^{*}\right)\right) I+L}{\lambda(\tilde{\xi}, \tilde{x})} d \tilde{\xi}+\int_{\tilde{\xi}_{\theta}\left(\tilde{x}+\Delta x^{*}\right)}^{\tilde{\xi}_{\hat{\lambda}}\left(\tilde{x}+\Delta x^{*}\right)} D r_{D} d \tilde{\xi}\right] .
\end{aligned}
$$

Totally diffentiating (A.3), we can compute

$$
\frac{d \tilde{\xi}_{\hat{\lambda}}(\tilde{x}+\Delta x)}{d \Delta x}=-\frac{2 \varepsilon \Gamma}{1-\delta+2 \varepsilon \Gamma}
$$

where $\Gamma$ is defined as

$$
\left.\Gamma \equiv \frac{\partial \hat{\lambda}(\tilde{\xi}+\Delta x)}{\partial \Delta x}\right|_{\tilde{\xi}=\tilde{\xi}_{\hat{\lambda}}(\tilde{x}+\Delta x)} .
$$

\footnotetext{
${ }^{1}$ The derivative $d \tilde{\xi}_{\theta}(\tilde{x}+\Delta x) / d \Delta x$ does not appear because $\left(\left(\tilde{\xi}_{\theta}(\tilde{x}+\Delta x)+\Delta x\right) I+L\right) / \lambda\left(\tilde{\xi}_{\theta}(\tilde{x}+\Delta x), \tilde{x}\right)=\left(\left(\tilde{\xi}_{\theta}(\tilde{x}+\Delta x)+\right.\right.$ $\Delta x) I+L) / \theta\left(\tilde{\xi}_{\theta}(\tilde{x}+\Delta x)+\Delta x\right)=D r_{D}$.
} 
Because we only care about $\Gamma$ for candidate values of $\tilde{x}+\Delta x$ in $\left(\xi_{l d}-\varepsilon, \xi_{u d}+\varepsilon\right)$, we know that $\hat{\lambda}$ is increasing on $\Delta x$ from Lemma 2 . Hence, $\Gamma$ is strictly positive.

Evaluating (A.7) at $\Delta x=\Delta x^{*}$ and rewriting $\tilde{x}+\varepsilon-\tilde{\xi}_{\hat{\lambda}}\left(\tilde{x}+\Delta x^{*}\right)=2 \varepsilon\left(\hat{\lambda}\left(\tilde{\xi}_{\hat{\lambda}}\left(\tilde{x}+\Delta x^{*}\right)+\Delta x\right)-\delta\right) /(1-\delta)$ from (A.3), the square-bracketed terms in the second line in (A.6), can be written as:

$$
\begin{aligned}
& \left.\frac{d \tilde{\xi}_{\hat{\lambda}}(\tilde{x}+\Delta x)}{d \Delta x}\right|_{\Delta x=\Delta x^{*}}\left(\tilde{\xi}_{\hat{\lambda}}\left(\tilde{x}+\Delta x^{*}\right)+\Delta x^{*}\right)+\left(\tilde{x}+\varepsilon-\tilde{\xi}_{\hat{\lambda}}\left(\tilde{x}+\Delta x^{*}\right)\right)= \\
& -\frac{2 \varepsilon}{1-\delta+2 \varepsilon \Gamma}\left[\Gamma \cdot\left(\tilde{\xi}_{\hat{\lambda}}\left(\tilde{x}+\Delta x^{*}\right)+\Delta x^{*}\right)-\left(\hat{\lambda}\left(\tilde{\xi}_{\hat{\lambda}}\left(\tilde{x}+\Delta x^{*}\right)+\Delta x\right)-\delta\right)\left(1+\frac{2 \varepsilon \Gamma}{1-\delta}\right)\right] .
\end{aligned}
$$

Recall that $\Gamma$ is defined in (A.8). Then, following the exact same calculations as in Lemma 5 in the paper, we get $\Gamma \cdot\left(\tilde{\xi}_{\hat{\lambda}}\left(\tilde{x}+\Delta x^{*}\right)+\Delta x^{*}\right)-\left(\hat{\lambda}\left(\tilde{\xi}_{\hat{\lambda}}\left(\tilde{x}+\Delta x^{*}\right)+\Delta x\right)-\delta\right)>0$. For small enough noise $\varepsilon$, the squarebracketed terms in (A.9) continue to be positive and, hence, (A.9) is negative overall.

Now, consider the square-bracketed terms in the third line in A.6, which can be written, by substituting the definition of $\lambda(\tilde{\xi}, \tilde{x})$, as:

$$
\int_{\tilde{x}-\varepsilon}^{\tilde{\xi}_{\theta}\left(\tilde{x}+\Delta x^{*}\right)} \frac{\left(\xi-\tilde{\xi}_{\hat{\lambda}}\left(\tilde{x}+\Delta x^{*}\right)\right) I+L}{\frac{1+\delta}{2}+\frac{(1-\delta)(\tilde{x}-\tilde{\xi})}{2 \varepsilon}} d \tilde{\xi}+\int_{\tilde{\xi}_{\theta}\left(\tilde{x}+\Delta x^{*}\right)}^{\tilde{\xi}_{\hat{\lambda}}\left(\tilde{x}+\Delta x^{*}\right)} D r_{D} d \tilde{\xi} .
$$

The first term in (A.10) can be made very close to zero for small enough noise, and because the second term is positive, (A.10) is positive.

Because (A.9) is negative and (A.10) is positive, (A.6) is positive overall for small enough, but nonvanishing noise $\varepsilon>0$. Hence, the threshold $x^{*}=\tilde{x}+\Delta x^{*}$ is unique.

\section{B Tools-augmented}

This section presents the problem of a tools-augmented planner who is endowed with the set of tools $\mathcal{T} \in \mathbb{T}$ and wants to replicate the social planner's allocations $\mathcal{C}_{s p}$, as a private equilibrium. The tools-augmented planner's problem is akin to a Ramsey planner's problem in the public finance literature.

We will consider two types of tools. First, restrictions on regulatory ratios denoted by $\mathcal{T}_{R}$. Second, Pigouvian taxes imposed directly on $B^{\prime} s$ payoffs and denoted by $\mathcal{T}_{P}$. For each $\mathcal{T}_{R}$, there is a regulatory constraint $R C\left(\mathcal{T}_{R}, \mathcal{C}\right) \geq 0$, which ties the tool with the endogenous variables $\mathcal{C}$, while for each $\mathcal{T}_{P}$, there is an additional term in $B$ 's utility, $\mathbb{U}_{B}\left(\mathcal{T}_{P}, C\right)$. It is important to note that the regulatory constraints, $R C$, are defined as inequalities, i.e., the planner can tighten them but not loosen them while there are no restrictions on Pigouvian taxes, which can be positive or negative. Let $\psi_{\mathcal{T}_{R}}$ be the multipliers that the banker in the private equilibrium assigns to constraint $R C\left(\mathcal{T}_{R}, \mathcal{C}\right) \geq 0$.

Under regulation, the optimization margins change to:

$$
\begin{gathered}
A A M_{\mathbb{T}}: A A M_{P E}+\sum_{\mathcal{T}}\left\{\psi_{\mathcal{T}_{R}}\left[\frac{\partial R C\left(\mathcal{T}_{R}, \mathcal{C}\right)}{\partial L}-\frac{\partial R C\left(\mathcal{T}_{R}, \mathcal{C}\right)}{\partial I}\right]+\frac{\partial \mathbb{U}_{B}\left(\mathcal{T}_{P}, \mathcal{C}\right)}{\partial L}-\frac{\partial \mathbb{U}_{B}\left(\mathcal{T}_{P}, \mathcal{C}\right)}{\partial I}\right\}=0, \\
C S M_{\mathbb{T}}: C S M_{P E}+\sum_{\mathcal{T}}\left\{\psi_{\mathcal{T}_{R}}\left[\frac{\partial R C\left(\mathcal{T}_{R}, \mathcal{C}\right)}{\partial E}-\frac{\partial R C\left(\mathcal{T}_{R}, \mathcal{C}\right)}{\partial D}\right]+\frac{\partial \mathbb{U}_{B}\left(\mathcal{T}_{P}, \mathcal{C}\right)}{\partial E}-\frac{\partial \mathbb{U}_{B}\left(\mathcal{T}_{P}, \mathcal{C}\right)}{\partial D}\right\}=0,
\end{gathered}
$$


$D I M_{\mathbb{T}}: D I M_{P E}+\sum_{\mathcal{T}}\left\{\psi_{\mathcal{T}_{R}}\left[\frac{\partial R C\left(\mathcal{T}_{R}, \mathcal{C}\right)}{\partial I}+\frac{\partial R C\left(\mathcal{T}_{R}, \mathcal{C}\right)}{\partial D}\right]+\frac{\partial \mathbb{U}_{B}\left(\mathcal{T}_{P}, \mathcal{C}\right)}{\partial I}+\frac{\partial \mathbb{U}_{B}\left(\mathcal{T}_{P}, \mathcal{C}\right)}{\partial D}\right\}=0$

We will show that in order to implement the equilibrium allocations of the social planner, denoted by $\mathcal{C}_{s p}$, it is not necessary to solve the full problem of the tools-augmented planner. Instead, it suffices that there are tools, $\mathcal{T}=\left\{\mathcal{T}_{R}, \mathcal{T}_{P}\right\}$, that first satisfy the regulatory constraints $R C\left(\mathcal{T}_{R}, \mathcal{C}_{s p}\right)=0$ at the planner's allocations, and, second, the margins in the associated equilibrium are the same as the margins of the planner. Essentially, this means that the additional terms in (B.1), (B.2), and (B.3) need to equal the wedges derived in (44), (45), and (46). In matrix form, this can be written as:

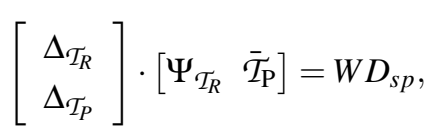

where $\Psi_{\mathcal{T}_{R}}$ is the $\mathcal{T}_{R} x 1$ vector of the multiplier on the $\mathcal{T}_{R}$ regulatory constraints, $W D_{s p}$ is the $3 x 1$ vector of the wedges in the three margins evaluated at the planner's equilibrium values, $\Delta_{\mathcal{T}_{R}} \equiv \Delta R C\left(\mathcal{T}_{R}, \mathcal{C}_{s p}\right)$ is the $3 x \mathcal{T}_{R}$ matrix of the partial derivatives of the relevant variables for each margin on the $\mathcal{T}_{R}$ regulatory constraints, $\overline{\mathcal{T}}_{P}$ is the $\mathcal{T}_{P} x 1$ vector of Pigouvian tools, and $\Delta_{\mathcal{T}_{P}}$ is the $3 x \mathcal{T}_{P}$ matrix of the coefficient on the tools $\mathcal{T}_{P}$ in the partial derivatives of the utility terms, i.e., $\Delta_{\mathcal{T}_{P}} \cdot \overline{\mathcal{T}}_{P} \equiv \Delta \mathbb{U}_{B}\left(\mathcal{T}, \mathcal{C}_{s p}\right)$.

Given that there are at most three distorted wedges in margins, only three independent tools are needed, i.e, $\# \mathcal{T}_{R}+\# \mathcal{T}_{P}=3$. First, consider that there are three tools $\mathcal{T}_{P}$ and none $\mathcal{T}_{R}$. As long as $\Delta_{\mathcal{T}_{P}}$ is invertible, three Pigouvian tools $\mathcal{T}_{P}$ are sufficient to implement the planner's solution, i.e., (B.4) has a solution even if regulatory-ratio tools $\mathcal{T}_{R}$ are not considered. Alternatively, the planner's allocations can be implemented with just three regulatory-ratio tools, if first, the matrix $\Delta_{\mathcal{T}_{R}}$ is invertible and, second, all elements in $\Psi_{\mathcal{T}_{R}}$ are positive. But, three regulatory-ratio tools (capital or liquidity) may not be linearly independent, because choosing two of them may replicate the value of the third. For example, a capital and a liquidity tool can be jointly binding, but two liquidity tools cannot. Additionally, some of resulting multipliers $\psi_{\mathcal{T}_{R}}$ may be negative, because the planner may want to encourage instead of restrict activity (recall that the regulatory constraints $R C$ are inequalities). Indeed, this is the case we study in section 4.3. For these reasons, we combine a capital and a liquidity tool with a (Pigouvian) subsidy on deposit interest expenses to implement the planner's allocations when savers are favored, while a capital tool is combined with (Pigouvian) deposit and lending subsidies when entrepreneurs are favored. When both regulatory-ratio and Pigouvian tools are used, it suffices that the matrix $\Delta_{\mathcal{T}} \equiv\left[\Delta_{\mathcal{T}_{R}} \Delta_{\mathcal{T}_{\mathbb{R}}}\right]^{\prime}$ is invertible in order to implement the planner's allocations.

We now show that (B.4) is a necessary and sufficient condition such that the social planner's solution described in section 3.1 can be decentralized as a private equilibrium by using regulatory tools $\mathcal{T}=\left\{\mathcal{T}_{R}, \mathcal{T}_{P}\right\} \in$ $\mathbb{T}$. The tools-augmented planner not only chooses optimally allocations $\mathcal{C}$, but also the level of tools $\mathcal{T} \in \mathbb{T}$ and the multipliers $\psi_{\mathcal{T}_{R}}$, which are the shadow values that the banker assigns to constraints $R C\left(\mathcal{T}_{R}, \mathcal{C}\right) \geq 0$ in the new equilibrium. Her problem is:

$$
\begin{gathered}
\max _{\mathcal{C}, \mathcal{T}, \Psi_{\mathcal{T}_{R}}} \mathbb{U}_{\mathrm{sp}}^{\mathbb{T}} \quad \text { s.t. } \mathscr{Y}(\mathcal{C})=0, R C\left(\mathcal{T}_{R}, \mathcal{C}\right) \geq 0, A A M_{\mathcal{T}}\left(\mathcal{T}_{R}, \mathcal{C}, \Psi_{\mathcal{T}}\right)=0, \operatorname{CSM}_{\mathcal{T}}\left(\mathcal{T}_{R}, \mathcal{C}, \Psi_{\mathcal{T}}\right)=0 \\
\operatorname{DIM}_{\mathcal{T}}\left(\mathcal{T}_{R}, \mathcal{C}, \Psi_{\mathcal{T}}\right)=0 .
\end{gathered}
$$

Note that the additional utility terms $\mathbb{U}_{B}\left(\mathcal{T}_{P}, \mathcal{C}\right)$ due to Pigouvian taxation tools do not appear in the utility that the tools-augmented planner maximizes, because she engages in lump-sum transfers of equal size. 
The first-order condition with respect to $\mathcal{C}$ (similar to first-order condition (43)) are:

$$
\begin{aligned}
& \frac{\partial \mathbb{U}_{B}}{\partial \mathcal{C}}+w_{S} \frac{\partial \mathbb{U}_{S}^{*}}{\partial \mathcal{C}}+w_{E} \frac{\partial \mathbb{U}_{E}^{*}}{\partial \mathcal{C}}+\sum_{\mathcal{Y}} \zeta_{\gamma} \frac{\partial \mathcal{Y}}{\partial \mathcal{C}} \\
+ & \sum_{\mathcal{T}_{R}} \zeta_{\mathcal{T}_{R}} \frac{\partial R C}{\partial \mathcal{C}}+\zeta_{A A M} \frac{\partial A A M_{\mathcal{T}}}{\partial \mathcal{C}}+\zeta_{C S M} \frac{\partial C S M_{\mathcal{T}}}{\partial \mathcal{C}}+\zeta_{D I M} \frac{\partial D I M_{\mathcal{T}}}{\partial \mathcal{C}}=0,
\end{aligned}
$$

where $\zeta_{\mathcal{T}_{R}}, \zeta_{A A M}, \zeta_{C S M}$ and $\zeta_{D I M}$ are the multipliers the tool-augmented planner assigns to regulatory constraints and the three regulation-distorted margins.

The first-order conditions with respect to the level of tools $\mathcal{T}_{R}$ and $\mathcal{T}_{P}$, respectively, are:

$$
\zeta_{\mathcal{T}_{R}} \frac{\partial R C}{\partial \mathcal{T}_{R}}+\zeta_{A A M} \frac{\partial A A M_{\mathcal{T}}}{\partial \mathcal{T}_{R}}+\zeta_{C S M} \frac{\partial C S M_{\mathcal{T}}}{\partial \mathcal{T}_{R}}+\zeta_{D I M} \frac{\partial D I M_{\mathcal{T}}}{\partial \mathcal{T}_{R}}=0
$$

and

$$
\zeta_{A A M} \frac{\partial A A M_{\mathcal{T}}}{\partial \mathcal{T}_{P}}+\zeta_{C S M} \frac{\partial C S M_{\mathcal{T}}}{\partial \mathcal{T}_{P}}+\zeta_{D I M} \frac{\partial D I M_{\mathcal{T}}}{\partial \mathcal{T}_{P}}=0
$$

Finally, choosing optimally the multipliers $\psi_{\mathcal{T}_{R}}$ yields:

$$
\zeta_{A A M} \frac{\partial A A M_{\mathcal{T}}}{\partial \psi_{\mathcal{T}_{R}}}+\zeta_{C S M} \frac{\partial C S M_{\mathcal{T}}}{\partial \psi_{\mathcal{T}_{R}}}+\zeta_{D I M} \frac{\partial D I M_{\mathcal{T}}}{\partial \psi_{\mathcal{T}_{R}}}=0
$$

The solutions of the social and tools-augmented planners coincide if the optimality conditions (43) and (B.6) coincide, i.e., if $\zeta_{A A M}=\zeta_{C S M}=\zeta_{D I M}=0$ and $\zeta_{\mathcal{T}_{R}}=0$ for all tools $\mathcal{T}_{R}$.

To prove sufficiency, note that augmenting (B.9) and (B.8) and representing them in combat form yields $\Delta_{\mathcal{T}}{ }^{\prime} \cdot\left[\zeta_{A A M} \zeta_{\text {CSM }} \zeta_{\text {DIM }}\right]^{\prime}=0$. Given that $\Delta_{\mathcal{T}}$ should be invertible for (B.4) to yield a solution, its transpose is also invertible, and the only solution is $\zeta_{A A M}=\zeta_{C S M}=\zeta_{D I M}=0$. Thus, all $\zeta_{\mathcal{T}_{R}}=0$ in (B.7) are also zero, and (43) and (B.6) coincide.

To prove necessity, suppose that (B.4) does not hold, i.e., $\Delta_{\mathcal{T}}$ is not invertible and some or all multipliers $\zeta_{\mathcal{T}_{R}}, \zeta_{A A M}, \zeta_{C S M}$ and $\zeta_{D I M}$ do not need to be zero. Using conditions (B.6), we can derive margins $A A M_{T A P}=$ $A A M_{S P}+A A M_{T A P, W D}, C S M_{T A P}=C S M_{S P}+C S M_{T A P, W D}$ and $D I M_{T A P}=D I M_{S P}+D I M_{T A P, W D}$ for the toolaugmented planner, where the wedges are linear combination of the multipliers $\zeta_{\mathcal{T}_{R}}, \zeta_{A A M}, \zeta_{C S M}$ and $\zeta_{D I M}$. The social planner's and tools-augmented planner's solutions coincide if wedges $A A M_{T A P, W D}, C S M_{T A P, W D}$, and $D I M_{T A P, W D}$ are all zero, which in principle is possible by varying $\zeta_{\mathcal{T}_{R}}$, $\zeta_{A A M}, \zeta_{C S M}$ and $\zeta_{D I M}$. However, equations (B.7), (B.8), and (B.9) remove as many degrees of freedom and are, thus, generically satisfied when all multipliers are zero-a contradiction.

\section{Expressions for margins and derivatives}

\section{C.1 Intermediation Margins in Private Equilibrium}

The first-order conditions (33) together with the four constraints in $\mathcal{Y}$ can be combined to characterize the private equilibrium as follows. If (33) gives an interior $r_{D}>1$, then it is used to determine $r_{D}$ as a function of all other variables in $C$; otherwise, set $r_{D}=1$. Then, use (2), (7), (9), and (32) to express (implicitly) $R_{D}$, $R_{I}, E$, and $\xi^{*}$ in terms of $I, L$ and $D$. The next step is to express the shadow values on the four constraints $\mathcal{Y}$ 
in terms of $I, L$, and $D$. The shadow value of funds is determined by the first-condition with respect to $E$,

$$
\psi_{B S}=W^{\prime}\left(e_{B}+D-I-L\right)
$$

where we have substituted $E=I+L-D$.

The shadow value on the deposit supply schedule can be obtained from (33) with respect to $R_{D}$, which yields

$$
\psi_{D S}=-\left(\frac{\partial \mathbb{U}_{B}}{\partial R_{D}}+\psi_{G G} \frac{\partial G G}{\partial R_{D}}\right) \frac{\partial D S^{-1}}{\partial R_{D}} .
$$

The choice of $R_{D}$ matters for the banker via the effect on profits and on the run dynamics. The shadow value determined in (C.2) captures the sum of these effects as the deposit rate moves along the deposit supply schedule.

The shadow value on the loan demand schedule can be obtained from (33) with respect to $R_{I}$, which yields

$$
\psi_{L D}=-\left(\frac{\partial \mathbb{U}_{B}}{\partial R_{I}}+\psi_{G G} \frac{\partial G G}{\partial R_{I}}\right) \frac{\partial L D^{-1}}{\partial R_{I}} .
$$

Similar to (C.2), condition (C.3) says that the shadow value on the loan demand is measured by how a change in the loan rate along the loan demand schedule matters via the effect on profits and on the run dynamics

Equivalently, combining (33) for $\mathcal{C}=\xi^{*},(\mathrm{C} .2)$ and (C.3), the shadow value on the global game constraint is given by

$$
\psi_{G G}=-\frac{d \mathbb{U}_{B}}{d \xi^{*}} \cdot \frac{d G G^{-1}}{d \xi^{*}},
$$

where $d \mathbb{U}_{B} / d \xi^{*}$ is the total effect of the run threshold $\xi^{*}$ on banker's utility, which captures the partial direct effect $-\partial \mathbb{U}_{B} / \partial \xi^{*}$ in (C.23) — and the partial indirect effects via the deposit and loan rate, in (C.23) and (C.23), respectively:

$$
\frac{d \mathbb{U}_{B}}{d \xi^{*}}=\left(\frac{\partial \mathbb{U}_{B}}{\partial \xi^{*}}-\frac{\partial \mathbb{U}_{B}}{\partial R_{D}} \frac{\partial D S^{-1}}{\partial R_{D}} \frac{\partial D S}{\partial \xi^{*}}-\frac{\partial \mathbb{U}_{B}}{\partial R_{I}} \frac{\partial L D^{-1}}{\partial R_{I}} \frac{\partial L D}{\partial \xi^{*}}\right) .
$$

Similarly, $d G G / d \xi^{*}$ is the total effect of the run threshold $\xi^{*}$ on the utility differential determining the run behavior, which captures the partial direct effect on $G G$ in (C.39) reported in Appendix C.3, and the partial indirect effects via the deposit and loan rate in (C.47) and (C.55) reported in Appendix C.3,:

$$
\frac{d G G}{d \xi^{*}}=\frac{\partial G G}{\partial \xi^{*}}-\frac{\partial G G}{\partial R_{D}} \frac{\partial D S^{-1}}{\partial R_{D}} \frac{\partial D S}{\partial \xi^{*}}-\frac{\partial G G}{\partial R_{I}} \frac{\partial L D^{-1}}{\partial R_{I}} \frac{\partial L D}{\partial \xi^{*}} .
$$

Overall, $\psi_{G G}$ measures the effect of a change in the run threshold, which is consistent with optimal run behavior, i.e., along the global game constraint, on $B$ 's welfare.

Combining (33) with respect to $L$ and $I$ and substituting in (C.2), (C.3), and (C.4), we obtain the asset 
allocation margin in the private equilibrium $\left(A A M_{P E}\right)$ :

$$
\begin{gathered}
\overbrace{\frac{\partial \mathbb{U}_{B}}{\partial L}-\frac{\partial \mathbb{U}_{B}}{\partial I}-\frac{\partial \mathbb{U}_{B}}{D} \frac{\partial D S^{-1}}{\partial R_{D}}\left(\frac{\partial D S}{\partial L}-\frac{\partial D S}{\partial I}\right)-\frac{\partial \mathbb{U}_{B}}{\partial R_{I}} \frac{\partial L D^{-1}}{\partial R_{I}}\left(\frac{\partial L D}{\partial L}-\frac{\partial L D}{\partial I}\right)}^{\text {Effect of asset mix on } \mathbb{U}_{B} \text { via bank profits: } d \mathbb{U}_{B} / d L-d \mathbb{U}_{B} / d I} \\
+\underbrace{\left(\frac{\partial \mathbb{U}_{B}}{\partial \xi^{*}}-\frac{\partial \mathbb{U}_{B}}{\partial R_{D}} \frac{\partial D S^{-1}}{\partial R_{D}} \frac{\partial D S}{\partial \xi^{*}}-\frac{\partial \mathbb{U}_{B}}{\partial R_{I}} \frac{\partial L D^{-1}}{\partial R_{I}} \frac{\partial L D}{\partial \xi^{*}}\right)}_{\text {Effect on } \mathbb{U}_{B} \text { via } \xi^{*}: d \mathbb{U}_{B} / d \xi^{*}} \cdot \underbrace{\left(\frac{\partial \xi^{*}}{\partial L}-\frac{d \xi^{*}}{d I}\right)}_{\text {Effect of asset mix on } \xi^{*}}=0,
\end{gathered}
$$

where $d \xi^{*} / d L$ and $d \xi^{*} / d I$ are obtained from total differentiation of (32), hence

$$
\frac{d \xi^{*}}{d L}-\frac{d \xi^{*}}{d I}=-\left[\frac{\partial G G}{\partial L}-\frac{\partial G G}{\partial I}-\frac{\partial G G}{\partial R_{D}} \frac{\partial D S^{-1}}{\partial R_{D}}\left(\frac{\partial D S}{\partial L}-\frac{\partial D S}{\partial I}\right)-\frac{\partial G G}{\partial R_{I}} \frac{\partial L D^{-1}}{\partial R_{I}}\left(\frac{\partial L D}{\partial L}-\frac{\partial L D}{\partial I}\right)\right] \cdot{\frac{d G G^{-1}}{d \xi^{*}}}_{(\mathrm{C} 8)}^{8}
$$

The asset allocation margin in (C.7) captures the decision to substitute a unit of loans with a unit of liquid assets. The banker in the private equilibrium weighs the effect of the change in the asset mix on the bank profitability (the first line) and on the run threshold, which determines run-risk (the second line). The asset mix matters for bank profits because of portfolio effects (first two terms in first line), but also because of the way it influences the profit margin via the loan rate and deposit rates (remaining terms in first line). The latter (general equilibrium) effect via rates captures how the asset mix matters for the loan rate or deposit rate that entrepreneurs and depositors are willing to accept. Similarly, the asset mix changes the payoffs governing the run dynamics directly and indirectly via the loan and deposit rates (captured by (C.8)), which in turn affect the run threshold influencing $B$ 's welfare directly and indirectly via the loan and deposit rates.

Similarly, combining (33) with respect to $E$ and $D$ and substituting in (C.2), (C.3), and (C.4), we obtain the capital structure margin in the private equilibrium $\left(C S M_{P E}\right)$ :

$$
\begin{gathered}
\overbrace{\frac{\partial \mathbb{U}_{B}}{\partial E}-\frac{\partial \mathbb{U}_{B}}{\partial D}+\frac{\partial \mathbb{U}_{B}}{D} \frac{\partial D S^{-1}}{\partial R_{D}} \frac{\partial D S}{\partial D}+\frac{\partial \mathbb{U}_{B}}{\partial R_{I}} \frac{\partial L D^{-1}}{\partial R_{I}} \frac{\partial L D}{\partial D}}^{\text {Effect of liabilities mix on } \mathbb{U}_{B} \text { via bank profits: } d \mathbb{U}_{B} / d E-d \mathbb{U}_{B} / d D} \\
-\underbrace{\left(\frac{\partial \mathbb{U}_{B}}{\partial \xi^{*}}-\frac{\partial \mathbb{U}_{B}}{\partial R_{D}} \frac{\partial D S^{-1}}{\partial R_{D}} \frac{\partial D S}{\partial \xi^{*}}-\frac{\partial \mathbb{U}_{B}}{\partial R_{I}} \frac{\partial L D^{-1}}{\partial R_{I}} \frac{\partial L D}{\partial \xi^{*}}\right)}_{\text {Effect on } \mathbb{U}_{B} \text { via } \xi^{*}: d \mathbb{U}_{B} / d \xi^{*}} \cdot \underbrace{\frac{d \xi^{*}}{d D}}_{\begin{array}{c}
\text { Effect of liabilities } \\
\text { mix on } \xi^{*}
\end{array}}=0,
\end{gathered}
$$

where

$$
\frac{d \xi^{*}}{d D}=-\left[\frac{\partial G G}{\partial D}-\frac{\partial G G}{\partial R_{D}} \frac{\partial D S^{-1}}{\partial R_{D}} \frac{\partial D S}{\partial D}-\frac{\partial G G}{\partial R_{I}} \frac{\partial L D^{-1}}{\partial R_{I}} \frac{\partial L D}{\partial D}\right] \cdot{\frac{d G G^{-1}}{d \xi^{*}}}^{-}
$$

Finally, combining (33) with respect to $I$ and $D$ and substituting in (C.2), (C.3), and (C.4), we 
obtain the deposit lending margin in the private equilibrium $\left(D I M_{P E}\right)$ :

$$
\begin{gathered}
\overbrace{\frac{\partial \mathbb{U}_{B}}{\partial I}+\frac{\partial \mathbb{U}_{B}}{\partial D}-\frac{\partial \mathbb{U}_{B}}{D} \frac{\partial D S^{-1}}{\partial R_{D}}\left(\frac{\partial D S}{\partial I}+\frac{\partial D S}{\partial D}\right)-\frac{\partial \mathbb{U}_{B}}{\partial R_{I}} \frac{\partial L D^{-1}}{\partial R_{I}}\left(\frac{\partial L D}{\partial I}+\frac{\partial L D}{\partial D}\right)}^{\text {Effect of deposit funded loans on } \mathbb{U}_{B} \text { via bank profits: } d \mathbb{U}_{B} / d I+d \mathbb{U}_{B} / d D} \\
+\underbrace{\left(\frac{\partial \mathbb{U}_{B}}{\partial \xi^{*}}-\frac{\partial \mathbb{U}_{B}}{\partial R_{D}} \frac{\partial D S^{-1}}{\partial R_{D}} \frac{\partial D S}{\partial \xi^{*}}-\frac{\partial \mathbb{U}_{B}}{\partial R_{I}} \frac{\partial L D^{-1}}{\partial R_{I}} \frac{\partial L D}{\partial \xi^{*}}\right)}_{\text {Effect on } \mathbb{U}_{B} \text { via } \xi^{*}: d \mathbb{U}_{B} / d \xi^{*}} \cdot \underbrace{\left(\frac{d \xi^{*}}{d I}+\frac{d \xi^{*}}{d D}\right)}_{\begin{array}{c}
\text { Effect of deposit funded } \\
\text { loans on } \xi^{*}
\end{array}}=0,
\end{gathered}
$$

where

$$
\frac{d \xi^{*}}{d I}+\frac{d \xi^{*}}{d D}=-\left[\frac{\partial G G}{\partial I}+\frac{\partial G G}{\partial D}-\frac{\partial G G}{\partial R_{D}} \frac{\partial D S^{-1}}{\partial R_{D}}\left(\frac{\partial D S}{\partial I}+\frac{\partial D S}{\partial D}\right)-\frac{\partial G G}{\partial R_{I}} \frac{\partial L D^{-1}}{\partial R_{I}}\left(\frac{\partial L D}{\partial I}+\frac{\partial L D}{\partial D}\right)\right] \cdot{\frac{d G G^{-1}}{d \xi^{*}}}^{.}
$$

Note that, expanding the first two terms in (C.11), we get that

$$
\frac{\partial \mathbb{U}_{B}}{\partial I}+\frac{\partial \mathbb{U}_{B}}{\partial D}=\omega\left\{\left[(1-q)-\delta r_{D} \log \left(\bar{\xi} / \xi^{*}\right) / \Delta_{\xi}\right] R_{I}-(1-q)(1-\delta) R_{D}\right\},
$$

where $q$ is the run probability. Hence, the deposit lending margin can be proxied by the spread between the loan rate, $R_{I}$, and the late deposit rate, $R_{D}$.

The three margins pin down the three free variables $I, L$, and $D$. The remaining variables, $E, \xi^{*}$, $R_{I}$, and $R_{D}$, are implicitly functions of the three free variables via constraints (9), (32), (7) and (2), which are always binding in equilibrium. Hence, there are three degrees of freedom and the private equilibrium is characterized by (C.7), (C.9) and (C.11).

\section{C.2 Intermediation Margins in Social Planner's Equilibrium}

As for the private equilibrium, we can use the first-order conditions (43) in the planning problem together with the four constraints in $\mathcal{Y}$ to characterize the planning allocations. In particular, we use the first-order condition with respect to $r_{D}$ to determine its value, and (2), (7), (9), and (32) to express (implicitly) $R_{D}, R_{I}, E$, and $\xi^{*}$ in terms of $I, L$, and $D$. As discussed, we consider a planner that respects the deposit supply and loan demand schedule, because we want to focus on regulation to affect bank's behavior. See section D.8 for a more powerful planner, who can levy distortionary taxes to affect the private deposit supply and loan demand schedules.

Up to this point everything is analogous to the characterization of PE allocations in section C.2. But, the planner also cares about the direct effect on $S$ and $E$ welfare as captured in the social welfare function (38). This influences the functional form of the Lagrange multipliers on constraints $\mathcal{Y}$, which are denoted by $\zeta_{y}$ instead of $\psi y$.

The functional forms of $\zeta_{B S}, \zeta_{D S}$, and $\zeta_{L D}$ are the same as for $\psi_{B S}, \psi_{D S}$, and $\psi_{L D}$ given by (C.1), (C.2) and (C.3), with the exception that the latter two are functions of $\zeta_{G G}$ instead of $\psi_{G G}$. The reason is that $E, R_{D}$, and $R_{I}$ do not appear directly in (38). Note, this does not mean that the 
equilibrium values of these Lagrange multipliers are the same in the private and planning solutions. But, the multiplier $\zeta_{G G}$ on constraint (32) will have a different functional form compared to (C.4), because $\xi^{*}$ appears in the indirect utilities:

$$
\begin{aligned}
\zeta_{G G} & =-\left(\frac{\partial \mathbb{U}_{B}}{\partial \xi^{*}}-\frac{\partial \mathbb{U}_{B}}{\partial r_{3}^{D}} \frac{\partial D S^{-1}}{\partial r_{3}^{D}} \frac{\partial D S}{\partial \xi^{*}}-\frac{\partial \mathbb{U}_{B}}{\partial r^{I}} \frac{\partial L D^{-1}}{\partial r^{I}} \frac{\partial L D}{\partial \xi^{*}}+w_{S} \frac{\partial \mathbb{U}_{S}^{*}}{\partial \xi^{*}}+w_{E} \frac{\partial \mathbb{U}_{E}^{*}}{\partial \xi^{*}}\right) \frac{d G G^{-1}}{d \xi^{*}} \\
& =\psi_{G G}-\left(w_{S} \frac{\partial \mathbb{U}_{S}^{*}}{\partial \xi^{*}}+w_{E} \frac{\partial \mathbb{U}_{E}^{*}}{\partial \xi^{*}}\right) \frac{d G G^{-1}}{d \xi^{*}} \\
& =\psi_{G G}\left[1+\left(w_{S} \frac{\partial \mathbb{U}_{S}^{*}}{\partial \xi^{*}}+w_{E} \frac{\partial \mathbb{U}_{E}^{*}}{\partial \xi^{*}}\right) \frac{d \mathbb{U}_{B}}{d \xi^{*}}\right] .
\end{aligned}
$$

Because $\partial \mathbb{U}_{S}^{*} / \partial \xi^{*}=-\left[V(D)-V^{\prime}(D) D\right] \Delta_{\xi}<0, \partial \mathbb{U}_{E}^{*} / \partial \xi^{*}=-\left[c^{\prime}(I) I-c(I)\right] \Delta_{\xi}<0$, and, from (C.5), $d \mathbb{U}_{B} / d \xi^{*}<0,{ }^{2}$ internalizing the run consequences makes the Lagrange multiplier on the constraint (32) higher (when evaluated at the PE allocations). We can now derive the wedges between the private and social margins.

Combining (43) with respect to $L$ and $I$ together with (34), we can derive the following wedge in the Asset Allocation Margin (also reported in (44)):

$$
A A M_{W D}=\underbrace{\left(w_{S} \frac{\partial \mathbb{U}_{S}^{*}}{\partial \xi^{*}}+w_{E} \frac{\partial \mathbb{U}_{E}^{*}}{\partial \xi^{*}}\right) \cdot\left(\frac{\partial \xi^{*}}{\partial L}-\frac{\partial \xi^{*}}{\partial I}\right)}_{\text {Run consequences from asset allocation }}-\underbrace{w_{E}(1-q) c^{\prime \prime}(I) I}_{\text {Surplus to E from additional } I}
$$

As discussed, the first term in (C.15) captures how a shift in the asset allocation from loans to liquid asset holdings affects savers' and entrepreneurs' welfare via its effect on the run probability. Both savers and entrepreneurs are worse off when run-risk goes up, i.e., $\partial \mathbb{U}_{S}^{*} / \partial \xi^{*}<0$ and $\partial \mathbb{U}_{E}^{*} / \partial \xi^{*}<0$. Thus, if run-risk decreases when the asset allocation shifts towards liquid assets, then the planner would want a more liquid asset mix. The second term in (C.15) captures the surplus created for the entrepreneur from an additional unit of investment. This term is negative (including the minus sign) if the planner puts weight on $E$ and if $E$ extracts some surplus to start with, i.e., $c^{\prime \prime}>0$, which is true for a strictly convex function.

Examining the run consequences in more detail the term $\partial \xi^{*} / \partial L-\partial \xi^{*} / \partial I$ captures exactly the effects of substituting a unit of liquid assets with a unit of loans on the probability of a run and is given by (C.8). If it is negative, correcting the run consequences requires a more liquid asset mix. The first and third terms inside the bracket are unambiguously positive, respectively. The first term captures the direct effect of shifting the asset allocation towards more liquid assets on the incentive to run. Combining (C.36) and (C.35) we get that $\partial G G / \partial L-\partial G G / \partial I=\partial G G / \partial \ell$, which is positive as we prove in Proposition 4 (recall that $\ell \equiv L /(I+L)$ is the share of liquid assets in the asset

\footnotetext{
${ }^{2} \partial \mathbb{U}_{B} / \partial \xi^{*}<0$ from (C.23); $\partial \mathbb{U}_{B} / \partial R_{D}<0$ from (C.26); $\partial D S / D>0$ from (C.50); $\partial D S / \partial D S / \xi^{*}<0$ from (C.47); $\partial \mathbb{U}_{B} / \partial R_{I}>0$ from (C.24); $\partial L D / \partial R_{I}<0$ from (C.56); $\partial L D / \partial \xi^{*}$ from (C.55) can be positive or negative, so the effect through the loan demand cannot be unambiguously determined. However, in our examples, the first two terms dominate, and overall, a higher run threshold reduces banker's utility all else being equal.
} 
portfolio). Intuitively, a more liquid asset portfolio (directly) decreases the incentives to run. The third term captures the indirect effect via the loan rate. In particular, $\partial G G / \partial R_{I}>0$ from (C.32) and (C.40), $\partial L D / \partial R_{I}=-\omega \int_{\xi^{*}}^{\bar{\xi}}(1-y) I d \xi / \Delta_{\xi}<0$ from (C.56), and, combining (C.51) and (C.52), we get that $\partial L D / \partial L-\partial L D / \partial I=\omega\left[A-R_{I}\right] \log \left(\bar{\xi} / \xi^{*}\right) / \Delta_{\xi}\left[L+I-\delta D r_{D}\right] / I^{2}-(1-q) c^{\prime \prime}(I)>0$. Thus, the third term in (C.8) - including the minus sign—is positive. Intuitively, a higher loan rate reduces the incentives to run, because it increases bank profits and, hence, the region where the banker decides to monitor. In turn, a more liquid asset portfolio increases the loan demand, because of convex investment costs and fewer loans being recalled, pushing up the loan rate entrepreneurs are willing to pay.

Finally, the second term captures the indirect effect via the late deposit rate. If we could show that this is always positive, then the whole expression would be negative given that $d G G / d \xi^{*}$ needs to be positive to have $\psi_{G G}>0$ in the private equilibrium. In particular, combining (C.44) and (C.43) we get that $\partial D S / \partial L-\partial D S / \partial I=q\left[1-\left(\xi^{*}+\underline{\xi}\right) / 2-\right] / D>0$, while $\partial D S / \partial R_{D}=\omega(1-q)(1-\delta)>0$ from (C.50). Intuitively, a more liquid asset portfolio increases the demand for deposits because it increases the probability of being repaid in a run, $\theta$, and, thus, pushes down the rate depositors demand. However, the effect of the deposit rate on the incentives to run, $\partial G G / \partial R_{D}$, could be unambiguously determined:

$$
\frac{\partial G G}{\partial R_{D}}=\underbrace{(\hat{\lambda}-\delta) \omega D}_{\begin{array}{c}
\text { Higher payoff } \\
\text { given monitoring }
\end{array}}+\underbrace{\frac{\partial \hat{\lambda}}{\partial R_{D}} \omega D R_{D}}_{\begin{array}{c}
\text { Lower chance } \\
\text { of monitoring }
\end{array}}=\omega D \frac{(\lambda-\delta) r_{D} R_{I}-(1-\delta) \xi^{*} R_{D}}{r_{D} R_{I}-\xi^{*} R_{D}} .
$$

In other words, a higher deposit rate increases the payoff from waiting given monitoring, which reduces the incentives to run, but also reduces the chances that monitoring takes place, i.e., $\partial \hat{\lambda} / \partial R_{D}<$ 0 from (C.34), which increases the incentives to run. We haven't been able to sign the overall effect analytically, but, in all the examples we have studied, we find that $\partial G G / \partial R_{D}>0$, or in other words a higher deposit rate reduces the run probability, all else being equal. ${ }^{3}$ This is an important and novel channel in our model.

Turning to the capital structure margin, we combine (43) with respect to $E$ and $D$ together with (35) to get the following wedge (also reported in (45)):

$$
C S M_{W D}=-\underbrace{\left(w_{S} \frac{\partial \mathbb{U}_{S}^{*}}{\partial \xi^{*}}+w_{E} \frac{\partial \mathbb{U}_{E}^{*}}{\partial \xi^{*}}\right) \frac{\partial \xi^{*}}{\partial D}}_{\text {Run consequences from liabilities mix }}+\underbrace{w_{R}\left[U^{\prime \prime}\left(e_{R}-D\right) D+(1-q) V^{\prime \prime}\left(D r_{D} D r_{D}^{2}\right]\right.}_{\text {Surplus to } \mathrm{S} \text { from additional } D}
$$

Similar to the wedge in the asset allocation margin, the wedge in the capital structure margin features two components. The first term captures how a shift in the liabilities mix from deposits to equity affects savers' and entrepreneurs' welfare via its effect on the run probability. Both savers and entrepreneurs are worse off when run-risk goes up. Thus, if run-risk decreases when the liabilities

\footnotetext{
${ }^{3}$ Recall that $\partial \xi^{*} / D=-(\partial G G / D) /\left(\partial G G / \partial \xi^{*}\right)$.
} 
mix shifts towards equity, then the planner would want lower leverage. ${ }^{4}$ The second term captures the surplus created for savers from an additional unit of deposits and is negative if the planner puts weigh on $S$ and if $S$ extracts some surplus to start with, which is true for strictly concave functions $U$ and $V$. In others words, shifting the capital structure away from deposits entails a welfare cost because of the lower surplus from deposit services to savers.

With respect to the run consequences, the term $\partial \xi^{*} / \partial D$ can be decomposed into a direct effect on the incentives to run and two indirect effects via the deposit and loan rate depicted in (C.10). Similar to above, the direct effect and the indirect effect via the loan rate can be unambiguously signed, but the indirect effect via the deposit rate cannot. In particular, expanding (C.37), we get $\partial G G / \partial D=-\omega R_{D}\left[\delta r_{D} R_{I}+(1-\delta) \xi^{*} R_{D}\right]-r_{D}\left(\theta^{*}-\delta\right)<0$, while $\partial D S / \partial D<0$ from (C.45), and $\partial L D / \partial D<0$ from (C.53). But, as mentioned, we find that $\partial G G / \partial R_{D}>0$, which means that the indirect effect via the deposit rate operates in the opposite direction compared with both the direct effect and indirect effect via the loan rate. Nevertheless, the indirect effect via the deposit rate does not dominate the other two effects and $\partial \xi^{*} / \partial D>0$, which means that run-risk increases when deposits go up, all else being equal.

Lastly, combining first-order condition (43) with respect to $I$ and $D$ together with (36), we obtain the following wedge in the deposit lending margin (also reported in (46):

$$
\begin{aligned}
& D I M_{W D}=\overbrace{\left(w_{S} \frac{\partial \mathbb{U}_{S}^{*}}{\partial \xi^{*}}+w_{E} \frac{\partial \mathbb{U}_{E}^{*}}{\partial \xi^{*}}\right) \cdot\left(\frac{\partial \xi^{*}}{\partial I}+\frac{\partial \xi^{*}}{\partial D}\right)}^{\text {Run consequences of boosting loans and deposits }} \\
& \underbrace{-w_{S}\left[U^{\prime \prime}\left(e_{S}-D\right) D+(1-q) V^{\prime \prime}\left(D r_{D}\right) D r_{D}^{2}\right]+w_{E}(1-q) c^{\prime \prime}(I) I}_{\text {Surplus to } S \text { and } E \text { from boosting loans and deposits }} .
\end{aligned}
$$

Similar to the other two wedges, (C.18) has a component that captures the consequences from boosting loans and deposits on the run probability and a component that captures the surplus created for $S$ and $E$. The latter is unambiguously positive as more deposits channeled to lending, increases the surplus to both savers and entrepreneurs. The impact of boosting loans and deposits on the run probability captured by $\partial \xi^{*} / \partial I+\partial \xi^{*} / \partial D$ (see (C.12) for the detailed expression) comprises of a direct effect as well as indirect effects via the deposit and loan rate, similar to the other two wedges. All three components are hard to sign analytically without knowing the ratio of lending to deposits, $I / D$, in the private equilibrium.

Overall, the planner balances the run consequences and the additional surpluses to savers and entrepreneurs when deciding how the asset allocation, the capital structure, and the level of deposit funded loans differ from the private equilibrium.

\footnotetext{
${ }^{4}$ Note that the equity capital choice does not directly enter the global game constraint $G G$. Thus, the effect of shifting the capital structure from deposit to equity is written as $\partial \xi^{*} / \partial E-\partial \xi^{*} / \partial D=-\partial \xi^{*} / \partial D$.
} 


\section{C.3 Derivatives}

This section reports the partial derivatives of the banker's utility $\mathbb{U}_{B}$ in (13), the monitoring threshold $\hat{\lambda}$ in (19), the global game constraint $G G$ in (32), the deposit supply schedule $D S$ in (2), the loan demand schedule $L D$ in (7), and the (outside) equity supply schedule $E S$ in (D.25), with respect to the choice variables in $\mathbb{C}$. When it is unambiguous, we also report the sign of the derivatives.

Partial derivatives $\partial \mathbb{U}_{B} / \partial C$.

$\frac{\partial \mathbb{U}_{B}}{\partial I}=\omega(1-q) R_{I}>0$.

$\frac{\partial \mathbb{U}_{B}}{\partial L}=\omega R_{I} \log \left(\bar{\xi} / \xi^{*}\right) / \Delta_{\xi}>0$.

$\frac{\partial \mathbb{U}_{B}}{\partial D}=-\omega\left[\delta r_{D} R_{I} \log \left(\bar{\xi} / \xi^{*}\right) / \Delta_{\xi}+(1-\delta)(1-q) R_{D}\right]<0$.

$\frac{\partial \mathbb{U}_{B}}{\partial E}=-W^{\prime}\left(e_{B}-E\right)<0$

$\frac{\partial \mathbb{U}_{B}}{\partial \xi^{*}}=-\left[\omega\left(\left(\xi^{*} I-\delta D r_{D}+L\right) / \xi^{*} R_{I}-(1-\delta) D R_{D}\right)-X\right] / \Delta_{\xi}<0$.

$\frac{\partial \mathbb{U}_{B}}{\partial R_{I}}=\omega\left[(1-q) I-\left(\delta D r_{D}-L\right) \log \left(\bar{\xi} / \xi^{*}\right) / \Delta_{\xi}\right]>0$.

$\frac{\partial \mathbb{U}_{B}}{\partial r_{D}}=-\omega \delta D R_{I} \log \left(\bar{\xi} / \xi^{*}\right) / \Delta_{\xi}<0$.

$\frac{\partial \mathbb{U}_{B}}{\partial R_{D}}=-\omega(1-\delta)(1-q) D<0$.

Partial derivatives $\partial \hat{\lambda} / \partial C$.

$\frac{\partial \hat{\lambda}}{\partial I}=\xi^{*} R_{I} /\left[D\left(r_{D} R_{I}-\xi^{*} R_{D}\right)\right]>0$

$\frac{\partial \hat{\lambda}}{\partial L}=R_{I} /\left[D\left(r_{D} R_{I}-\xi^{*} R_{D}\right)\right]>0$. 


$$
\frac{\partial \hat{\lambda}}{\partial D}=-\xi^{*} R_{D} /\left[D\left(r_{D} R_{I}-\xi^{*} R_{D}\right)\right]-\hat{\lambda} / D<0 .
$$

$$
\frac{\partial \hat{\lambda}}{\partial E}=0 .
$$

$$
\begin{aligned}
\frac{\partial \hat{\lambda}}{\partial \xi^{*}}= & {\left[I R_{I}-\left(D R_{D}+X / \omega\right)\right] /\left[D\left(r_{D} R_{I}-\xi^{*} R_{D}\right)\right] } \\
& +\hat{\lambda} R_{D} /\left[r_{D} R_{I}-\xi^{*} R_{D}\right]>0 .
\end{aligned}
$$

$$
\begin{aligned}
\frac{\partial \hat{\lambda}}{\partial R_{I}} & =\left(\xi^{*} I+L\right) /\left[D\left(r_{D} R_{I}-\xi^{*} R_{D}\right)\right] \\
& -\hat{\lambda} r_{D} /\left[r_{D} R_{I}-\xi^{*} R_{D}\right]>0 .
\end{aligned}
$$

$\frac{\partial \hat{\lambda}}{\partial r_{D}}=-\hat{\lambda} R_{I} /\left[r_{D} R_{I}-\xi^{*} R_{D}\right]<0$.

$$
\frac{\partial \hat{\lambda}}{\partial R_{D}}=-(1-\hat{\lambda}) \xi^{*} /\left[r_{D} R_{I}-\xi^{*} R_{D}\right]<0 .
$$

Partial derivatives $\partial G G / \partial C$.

$$
\begin{aligned}
& \frac{\partial G G}{\partial I}=\omega D R_{D} \frac{\partial \hat{\lambda}}{\partial I}-\int_{\xi^{*}}^{1} \frac{\xi^{*}}{\lambda} d \lambda>0 . \\
& \frac{\partial G G}{\partial L}=\omega D R_{D} \frac{\partial \hat{\lambda}}{\partial L}-\int_{\xi^{*}}^{1} \frac{1}{\lambda} d \lambda \gtrless 0 . \\
& \frac{\partial G G}{\partial D}=\omega D R_{D}\left[\frac{\partial \hat{\lambda}}{\partial D}+(\hat{\lambda}-\delta) / D\right]-\left(\theta^{*}-\delta\right) r_{D}<0 . \\
& \frac{\partial G G}{\partial E}=0 . \\
& \frac{\partial G G}{\partial \xi^{*}}=\omega D R_{D} \frac{\partial \hat{\lambda}}{\partial \xi^{*}}-\int_{\xi^{*}}^{1} \frac{I}{\lambda} d \lambda>0 .
\end{aligned}
$$




$$
\begin{aligned}
& \frac{\partial G G}{\partial R_{I}}=\omega D R_{D} \frac{\partial \hat{\lambda}}{\partial R_{I}}>0 . \\
& \frac{\partial G G}{\partial r_{D}}=\omega D R_{D} \frac{\partial \hat{\lambda}}{\partial r_{D}}-D\left(\theta^{*}-\delta\right)<0 . \\
& \frac{\partial G G}{\partial R_{D}}=\omega D R_{D} \frac{\partial \hat{\lambda}}{\partial R_{D}}+\omega D(\hat{\lambda}-\delta) \gtrless 0 .
\end{aligned}
$$

Partial derivatives $\partial D S / \partial C$.

$$
\begin{aligned}
\frac{\partial D S}{\partial I} & =\left[\beta \delta+\beta^{2}(1-\beta)\right] \cdot q \cdot \frac{\xi^{*}+\underline{\xi}}{2} \cdot \frac{1}{D}>0 . \\
\frac{\partial D S}{\partial L} & =\left[\beta \delta+\beta^{2}(1-\beta)\right] \cdot q \cdot \frac{1}{D}>0 . \\
\frac{\partial D S}{\partial D} & =U^{\prime \prime}\left(e_{S}-D\right)-\left[\beta \delta+\beta^{2}(1-\beta)\right] \cdot q \cdot\left(L+I \cdot \frac{\xi^{*}+\underline{\xi}}{2}\right) \frac{1}{D^{2}} \\
& +(1-q) V^{\prime \prime}\left(D r_{D}\right) r_{D}^{2}<0 .
\end{aligned}
$$

$$
\frac{\partial D S}{\partial E}=0 .
$$

$$
\begin{aligned}
\frac{\partial D S}{\partial \xi^{*}} & =\left\{\left[\beta \delta+\beta^{2}(1-\beta)\right] \frac{L+\xi^{*} I}{D}-\delta \beta r_{D}\right. \\
& \left.-(1-\delta) \beta^{2} \omega r_{D}-V^{\prime}\left(D r_{D}\right) r_{D}\right\} \Delta_{\xi}^{-1}<0 .
\end{aligned}
$$

$$
\begin{aligned}
& \frac{\partial D S}{\partial R_{I}}=0 . \\
& \frac{\partial D S}{\partial r_{D}}=(1-q)\left[\beta \delta+V^{\prime}\left(D r_{D}\right)+V^{\prime \prime}\left(D r_{D}\right) D r_{D}\right]>0 . \\
& \frac{\partial D S}{\partial R_{D}}=\omega \cdot \beta^{2}(1-\delta)(1-q)>0 .
\end{aligned}
$$


Partial derivatives $\partial L D / \partial C$.

$$
\begin{aligned}
& \frac{\partial L D}{\partial I}=\omega\left(A-R_{I}\right) \frac{\delta D r_{D}-L}{I^{2}} \frac{\log \left(\bar{\xi} / \xi^{*}\right)}{\Delta_{\xi}}-(1-q) c^{\prime \prime}(I) \lessgtr 0 . \\
& \frac{\partial L D}{\partial L}=\omega\left(A-R_{I}\right) \frac{1}{I} \frac{\log \left(\bar{\xi} / \xi^{*}\right)}{\Delta_{\xi}}>0 . \\
& \frac{\partial L D}{\partial D}=-\omega(A-(1+r)) \frac{\delta r_{D}}{I} \frac{\log \left(\bar{\xi} / \xi^{*}\right)}{\Delta_{\xi}}<0 . \\
& \frac{\partial L D}{\partial E}=0 \\
& \frac{\partial L D}{\partial \xi^{*}}=-\left[\omega\left(A-R_{I}\right) \frac{\xi^{*} I-\delta D r_{D}+L}{\xi^{*} I}-c^{\prime}(I)\right] \Delta_{\xi}^{-1} \gtrless 0 . \\
& \frac{\partial L D}{\partial R_{I}}=-\omega\left[(1-q)-\frac{\delta D r_{D}}{I} \frac{\log \left(\bar{\xi} / \xi^{*}\right)}{\Delta_{\xi}}\right]<0 . \\
& \frac{\partial L D}{\partial r_{D}}=-\omega(A-(1+r)) \frac{\delta D}{I} \frac{\log \left(\bar{\xi} / \xi^{*}\right)}{\Delta_{\xi}}<0 . \\
& \frac{\partial L D}{\partial R_{D}}=0
\end{aligned}
$$

Partial derivatives $\partial E S / \partial \tilde{C}$ for extension in section D.7.

$$
\begin{aligned}
& \frac{\partial E S}{\partial I}=\frac{1}{E+O} \omega(1-q) R_{I}>0 . \\
& \frac{\partial E S}{\partial L}=\frac{1}{E+O} \omega R_{I} \log \left(\bar{\xi} / \xi^{*}\right) / \Delta_{\xi}>0 . \\
& \frac{\partial E S}{\partial D}=-\frac{1}{E+O} \omega\left[\delta r_{D} R_{I} \log \left(\bar{\xi} / \xi^{*}\right) / \Delta_{\xi}+(1-\delta)(1-q) R_{D}\right]<0 .
\end{aligned}
$$




$$
\begin{aligned}
& \frac{\partial E S}{\partial E}=-\frac{1}{E+O} \int_{\xi^{*}}^{\bar{\xi}} D P S(\xi, \delta) \frac{d \xi}{\Delta_{\xi}}<0 . \\
& \frac{\partial E S}{\partial O}=P^{2} \cdot W^{\prime \prime}\left(e_{O}-P \cdot O\right)-\frac{1}{E+O} \int_{\xi^{*}}^{\bar{\xi}} D P S(\xi, \delta) \frac{d \xi}{\Delta_{\xi}}<0 . \\
& \frac{\partial E S}{\partial \xi^{*}}=-D P S\left(\xi^{*}, \delta\right) / \Delta_{\xi}<0 . \\
& \frac{\partial E S}{\partial R_{I}}=\frac{1}{E+O} \omega\left[(1-q) I-\left(\delta D r_{D}-L\right) \log \left(\bar{\xi} / \xi^{*}\right) / \Delta_{\xi}\right]>0 . \\
& \frac{\partial E S}{\partial r_{D}}=-\frac{1}{E+O} \omega \delta D R_{I} \log \left(\bar{\xi} / \xi^{*}\right) / \Delta_{\xi}<0 . \\
& \frac{\partial E S}{\partial R_{D}}=-\frac{1}{E+O} \omega(1-\delta)(1-q) D<0 . \\
& \frac{\partial E S}{\partial P}=P \cdot O \cdot W^{\prime \prime}\left(e_{O}-P \cdot O\right)-W^{\prime}\left(e_{O}-P \cdot O\right)<0 .
\end{aligned}
$$

\section{Extensions}

\section{D.1 Disciplining Role of Runs}

This section explores what is the role of the run in disciplining the banker. In particular, we compute the private equilibrium in which the banker does not internalize the effect of her actions on the run probability, i.e., $\psi_{G G}=0$ in (33). Table D.1 reports the results. The banker chooses allocations that result in higher run-risk and all agents are worse off, while capital and liquidity are much lower than in the socially optimal outcomes even when $E$ is favored. As expected, there is a much bigger scope for regulation if $B$ neglected her impact on run-risk and all agents could be made better off.

\section{D.2 Incomplete Deposit Contracts and Lack of Commitment}

This section studies the case of incomplete deposit contracts. First, we show how the wedges in the margins between the private and social solutions change. Second, we report how the numerical solution for the private equilibrium under incomplete contracts compares to the private and social outcomes discussed in section 3.2.

Under incomplete deposit contracts, the banker only internalizes the effect of terms specified 


\begin{tabular}{|c|c|c|c|c|c|}
\hline & \multirow{2}{*}{$\begin{array}{r}\mathrm{PE} \\
\psi_{G G}>0 \\
\end{array}$} & \multirow{2}{*}{$\begin{array}{r}\mathrm{PE} \\
\psi_{G G}=0 \\
\end{array}$} & \multicolumn{3}{|c|}{ SP for weights $\left(w_{E}, w_{S}\right)$} \\
\hline & & & $(0.00,0.20)$ & $(0.10,0.10)$ & $(0.20,0.00)$ \\
\hline$I$ & 0.862 & 0.827 & 0.785 & 0.873 & 0.906 \\
\hline$L$ & 0.052 & 0.000 & 0.221 & 0.060 & 0.000 \\
\hline$D$ & 0.875 & 0.803 & 0.962 & 0.894 & 0.867 \\
\hline E & 0.038 & 0.024 & 0.044 & 0.039 & 0.038 \\
\hline$R_{I}$ & 4.097 & 4.119 & 4.198 & 4.089 & 4.042 \\
\hline$R_{D}$ & 1.717 & 1.581 & 1.804 & 1.767 & 1.758 \\
\hline$q$ & 0.407 & 0.425 & 0.386 & 0.403 & 0.408 \\
\hline$\ell$ & 0.057 & 0.000 & 0.219 & 0.065 & 0.000 \\
\hline$k$ & 0.042 & 0.028 & 0.044 & 0.042 & 0.042 \\
\hline$R_{I}-(1-\delta) R_{D}$ & 3.239 & 3.328 & 3.296 & 3.205 & 3.163 \\
\hline$I+L$ & 0.914 & 0.827 & 1.006 & 0.933 & 0.906 \\
\hline$I-E$ & 0.824 & 0.803 & 0.741 & 0.834 & 0.867 \\
\hline$E($ Div $)$ & 0.745 & 0.703 & 0.755 & 0.747 & 0.743 \\
\hline$\Delta \mathbb{U}_{E}$ & - & $-1.11 \%$ & $-1.66 \%$ & $0.33 \%$ & $1.19 \%$ \\
\hline$\Delta \mathbb{U}_{S}$ & - & $-2.10 \%$ & $3.63 \%$ & $0.71 \%$ & $-0.30 \%$ \\
\hline$\Delta \mathbb{U}_{B}$ & - & $-0.89 \%$ & $-0.44 \%$ & $-0.05 \%$ & $-0.09 \%$ \\
\hline
\end{tabular}

Table D.1: Private equilibrium allocations when the banker does and does not internalize the effect of her action on run-risk versus Socially optimal solutions. The welfare changes are computed over the level of welfare in the private equilibrium where the banker internalizes run-risk, which is normalized to one for each agent.

in the deposit contract on the deposit supply. At minimum, these terms include the amount of deposits, $D$, and the deposit rates, $r_{D}$ and $R_{D}$. As a result, the banker would be tempted to deviate when choosing the rest of the balance sheet after she has entered into a deposit contract and received the deposits. The banker does understand that taking more risk increases the cost of raising deposits and would ideally want to promise depositors that she will behave prudently. But, after the deposit contract has been signed, the banker has an incentive to deviate towards lending more, holding fewer liquid assets, and raising less equity.

Depositors have rational expectations and ex ante require that the banker offers higher deposit rates to compensate for the anticipated risk-taking due to the lack of commitment. As a result, the deposit supply schedule has the same functional form to the benchmark environment. The difference in the private equilibrium comes from the fact that the banker will not include the effect of $I, L$ and $\xi^{*}$ on $D S$ in the respective first-order conditions, i.e., the respective (33) will not include the terms multiplied by $\psi_{D S}$.

The Lagrange multipliers $\psi_{D S}$ and $\psi_{L D}$ will have the same functional form derived in (C.2) and 
(C.3), respectively. However, the functional form of $\psi_{G G}$ will be different from the one in (C.4):

$$
\widehat{\psi}_{G G}=-\frac{\frac{\partial \mathbb{U}_{B}}{\partial \xi^{*}}-\frac{\partial \mathbb{U}_{B}}{\partial R_{I}} \frac{\partial L D}{\partial R_{I}}{ }^{-1} \frac{\partial L D}{\partial \xi^{*}}}{\frac{\partial G G}{\partial \xi^{*}}-{\frac{\partial G G}{\partial R_{I}}}_{\frac{\partial L D}{\partial R_{I}}}^{-1} \frac{\partial L D}{\partial \xi^{*}}} .
$$

So the difference between the multipliers in the social equilibrium, $\zeta_{G G}$ given by (C.14), and private equilibrium, $\widehat{\psi}_{G G}$ given by (D.1), does not only come from the presence of run consequences, but also from contract incompleteness:

$$
\begin{aligned}
& \zeta_{G G}-\widehat{\psi}_{G G}= \\
& \underbrace{-\left(w_{S} \frac{\partial \mathbb{U}_{S}^{*}}{\partial \xi^{*}}+w_{E} \frac{\partial \mathbb{U}_{E}^{*}}{\partial \xi^{*}}\right) \frac{d G G^{-1}}{d \xi^{*}}}_{\text {Run consequences }}+\underbrace{\left(\frac{\partial \mathbb{U}_{B}}{\partial R_{D}}+\widehat{\psi}_{G G} \frac{\partial G G}{\partial R_{D}}\right) \frac{\partial D S^{-1}}{\partial R_{D}} \frac{\partial D S}{\partial \xi^{*}} \frac{d G G^{-1}}{d \xi^{*}}}_{\text {Incomplete contract externality }} .
\end{aligned}
$$

Note that, under complete contracts, the difference between $\zeta_{G G}$ and $\psi_{G G}$, given by (C.4), is only due to the run consequences, i.e., the first term in (D.2).

The three expressions in (D.3), (D.4), and (D.5) below report the wedges in the three margins, separating the inefficiencies stemming from incomplete contracts.

The wedge is the asset allocation margin is:

$$
\begin{aligned}
& \widehat{A A M}_{W D}=\underbrace{\left(w_{S} \frac{\partial \mathbb{U}_{S}^{*}}{\partial \xi^{*}}+w_{E} \frac{\partial \mathbb{U}_{E}^{*}}{\partial \xi^{*}}\right)\left(\frac{\partial \xi^{*}}{\partial L}-\frac{\partial \xi^{*}}{\partial I}\right)}_{\text {Run consequences from asset allocation }}-\underbrace{w_{E}(1-q) c^{\prime \prime}(I) I}_{\text {Surplus to E from additional } I} \\
& +\left(\frac{\partial \mathbb{U}_{B}}{\partial R_{D}}+\widehat{\psi}_{G G} \frac{\partial G G}{\partial R_{D}}\right) \frac{\partial D S^{-1}}{\partial R_{D}} \frac{\partial D S}{\partial \xi^{*}} \\
& \underbrace{\left[\frac{\partial G G}{\partial L}-\frac{\partial G G}{\partial I}-\frac{\partial G G}{\partial R_{I}} \frac{\partial L D^{-1}}{\partial R_{I}}\left(\frac{\partial L D}{\partial L}-\frac{\partial L D}{\partial I}\right)\right] \frac{d G G^{-1}}{d \xi^{*}}}_{\text {(Indirect) Incomplete contract consequences }} \\
& \underbrace{-\left(\frac{\partial \mathbb{U}_{B}}{\partial R_{D}}+\zeta_{G G} \frac{\partial G G}{\partial R_{D}}\right) \frac{\partial D S^{-1}}{\partial R_{D}}\left(\frac{\partial D S}{\partial L}-\frac{\partial D S}{\partial I}\right)}_{\text {(Direct) Incomplete contract consequences }} .
\end{aligned}
$$

The terms in the first line are the same ones in (C.15) under complete deposit contracts. The second line captures the effect of the asset allocation on the run threshold, which in turn was not part of the deposit contract that the banker would internalize. The last line captures the direct effect of incomplete contracts on the asset allocation margin, i.e., the planner internalizes how the asset mix affects the deposit supply schedule.

Examining the direct effect first, note that $-\left(\partial \mathbb{U}_{B} / \partial R_{D}+\zeta_{G G} \partial G G / \partial R_{D}\right)\left(\partial D S / \partial R_{D}\right)^{-1}$ is the planner's Lagrange multiplier on the deposit supply schedule, which we expect to be positive as 
long as the planner wants to encourage the supply of deposits by offering a higher deposit rate. Also, we have shown that $(\partial D S / \partial I-\partial D S / \partial L)<0$, which means that having a less liquid asset mix will adversely affect the supply of deposits. The planner internalizes this, but the banker may have an incentive to deviate and take more asset risk after the deposit contract has been signed. The overall direct effect is negative, which means the planner (and a banker that can commit) would like to implement a more liquid asset allocation.

Turning to the indirect externality, $-\left(\partial \mathbb{U}_{B} / \partial R_{D}+\widehat{\psi}_{G G} \partial G G / \partial R_{D}\right)\left(\partial D S / \partial R_{D}\right)^{-1}$ is the multiplier on $D S$ in the private problem, which we expect to be positive for the same reasons as above. The overall effect from all the terms is negative (see the benchmark model where we sign the other terms). This means that the planner (and a banker that can commit) would, similarly to the direct effect, also want a more liquid asset allocation. Hence, the incomplete contract externality results in more asset risk in private allocations.

Similarly, the wedges for the capital structure becomes:

$$
\begin{gathered}
\widehat{C S M}_{W D}=-\underbrace{\left(w_{S} \frac{\partial \mathbb{U}_{S}^{*}}{\partial \xi^{*}}+w_{E} \frac{\partial \mathbb{U}_{E}^{*}}{\partial \xi^{*}}\right) \frac{\partial \xi^{*}}{\partial D}}_{\text {Run consequences from liabilities mix }}+\underbrace{w_{R}\left[U^{\prime \prime}\left(e_{R}-D\right) D+(1-q) V^{\prime \prime}\left(D r_{D} D r_{D}^{2}\right]\right.}_{\text {Surplus to } S \text { from additional } D} \\
\underbrace{-\left(\frac{\partial \mathbb{U}_{B}}{\partial R_{D}}+\widehat{\Psi}_{G G} \frac{\partial G G}{\partial R_{D}}\right) \frac{\partial D S^{-1}}{\partial R_{D}} \frac{\partial D S}{\partial \xi^{*}}\left[\frac{\partial G G}{\partial D}-\frac{\partial G G}{\partial R_{I}} \frac{\partial L D^{-1}}{\partial R_{I}} \frac{\partial L D}{\partial D}-\frac{\partial G G}{\partial R_{D}} \frac{\partial D S^{-1}}{\partial R_{D}} \frac{\partial D S}{\partial D}\right] \frac{d G G^{-1}}{d \xi^{*}}}_{\text {(Indirect) Incomplete contract consequences }} .
\end{gathered}
$$

Note that in (D.4), the additional terms stem only from incomplete contracts. 
Finally, the wedge in the deposit lending margin becomes:

$$
\begin{aligned}
& \widehat{D I M}_{W D}=\overbrace{\left(w_{S} \frac{\partial \mathbb{U}_{S}^{*}}{\partial \xi^{*}}+w_{E} \frac{\partial \mathbb{U}_{E}^{*}}{\partial \xi^{*}}\right)\left(\frac{\partial \xi^{*}}{\partial I}+\frac{\partial \xi^{*}}{\partial D}\right)}^{\text {Run consequences from boosting loans and deposits }} \\
& \underbrace{-w_{S}\left[U^{\prime \prime}\left(e_{S}-D\right) D+(1-q) V^{\prime \prime}\left(D r_{D}\right) D r_{D}^{2}\right]+w_{E}(1-q) c^{\prime \prime}(I) I}_{\text {Surplus to } S \text { and } E \text { from boosting loans and deposits }} \\
& +\left(\frac{\partial \mathbb{U}_{B}}{\partial R_{D}}+\widehat{\psi}_{G G} \frac{\partial G G}{\partial R_{D}}\right) \frac{\partial D S^{-1}}{\partial R_{D}} \frac{\partial D S}{\partial \xi^{*}} \\
& \underbrace{\left[\frac{\partial G G}{\partial I}+\frac{\partial G G}{\partial D}-\frac{\partial G G}{\partial R_{I}} \frac{\partial L D^{-1}}{\partial R_{I}}\left(\frac{\partial L D}{\partial I}+\frac{\partial L D}{\partial D}\right)-\frac{\partial G G}{\partial R_{D}} \frac{\partial D S^{-1}}{\partial R_{D}} \frac{\partial D S}{\partial D}\right] \frac{d G G^{-1}}{d \xi^{*}}}_{\text {(Indirect) Incomplete contract consequences }} \\
& \underbrace{-\left(\frac{\partial \mathbb{U}_{B}}{\partial R_{D}}+\zeta_{G G} \frac{\partial G G}{\partial R_{D}}\right) \frac{\partial D S}{\partial R_{D}} \frac{\partial D S}{\partial I}}_{\text {(Direct) Incomplete contract consequences }}
\end{aligned}
$$

Along the same lines, the direct incomplete contract externality in (D.5) is only due to the choice of I.

Overall, the consequences from incomplete contracts are an additional source of divergence between private and social margins. Table D. 2 compares the private equilibrium under incomplete deposit contracts to both the private equilibrium under complete contracts and the socially optimal allocations. Comparing the private equilibria under complete and incomplete contracts, we can see that the banker has an incentive to choose a less liquid asset portfolio and more leveraged capital structure. The inability to commit, results in higher run-risk, and the banker needs to cut deposittaking in order to sustain a not-too-low profit margin. All agents are worse off. As a result, the planner cannot only fix the run consequences, but also the inefficiencies arising from incomplete deposit contracts. In other words, the planner can "enable" the banker to commit, which can be beneficial for all agents including the banker.

\section{D.3 Loan Market and Price-Taking Behavior}

This section presents the private equilibrium outcomes when the banker acts as a price-taker in the loan market, i.e., she takes $R_{I}$ as given and does not internalize the effect of the other choices in $C$ on the loan demand schedule (7). Technically, this means that the first-order conditions (33) in the private equilibrium should not include the terms multiplied by $\psi_{L D}$. This would introduce an additional reason why the privately and socially optimal allocations diverge on top of the run consequences and surplus considerations present in the three wedges in (44), (45), and (46). Indeed, one can derive expressions similar to (D.3), (D.4), and (D.5) where, instead of the terms for the incomplete deposit contract consequences, there would be terms capturing the consequences from 


\begin{tabular}{|c|c|c|c|c|c|}
\hline & \multirow{2}{*}{$\begin{array}{r}\mathrm{PE} \\
\text { Complete }\end{array}$} & \multirow{2}{*}{$\begin{array}{r}\mathrm{PE} \\
\text { Incomplete }\end{array}$} & \multicolumn{3}{|c|}{ SP for weights $\left(w_{E}, w_{S}\right)$} \\
\hline & & & $(0.00,0.20)$ & $(0.10,0.10)$ & $(0.20,0.00)$ \\
\hline$I$ & 0.862 & 0.825 & 0.785 & 0.873 & 0.906 \\
\hline$L$ & 0.052 & 0.000 & 0.221 & 0.060 & 0.000 \\
\hline$D$ & 0.875 & 0.797 & 0.962 & 0.894 & 0.867 \\
\hline$E$ & 0.038 & 0.028 & 0.044 & 0.039 & 0.038 \\
\hline$R_{I}$ & 4.097 & 4.121 & 4.198 & 4.089 & 4.042 \\
\hline$R_{D}$ & 1.717 & 1.550 & 1.804 & 1.767 & 1.758 \\
\hline$q$ & 0.407 & 0.424 & 0.386 & 0.403 & 0.408 \\
\hline$\ell$ & 0.057 & 0.000 & 0.219 & 0.065 & 0.000 \\
\hline$k$ & 0.042 & 0.035 & 0.044 & 0.042 & 0.042 \\
\hline$R_{I}-(1-\delta) R_{D}$ & 3.239 & 3.346 & 3.296 & 3.205 & 3.163 \\
\hline$I+L$ & 0.914 & 0.825 & 1.006 & 0.933 & 0.906 \\
\hline$I-E$ & 0.824 & 0.797 & 0.741 & 0.834 & 0.867 \\
\hline$E(D i v)$ & 0.745 & 0.714 & 0.755 & 0.747 & 0.743 \\
\hline$\Delta \mathbb{U}_{E}$ & - & $-1.14 \%$ & $-1.66 \%$ & $0.33 \%$ & $1.19 \%$ \\
\hline$\Delta \mathbb{U}_{S}$ & - & $-2.12 \%$ & $3.63 \%$ & $0.71 \%$ & $-0.30 \%$ \\
\hline$\Delta \mathbb{U}_{B}$ & - & $-0.85 \%$ & $-0.44 \%$ & $-0.05 \%$ & $-0.09 \%$ \\
\hline
\end{tabular}

Table D.2: Private equilibrium allocations under complete and incomplete deposit contracts versus Socially optimal solutions. The welfare changes are computed over the level of welfare in the private equilibrium under complete contracts, which is normalized to one for each agent.

pricing taking behavior in the loan market.

Table D. 3 compares the private equilibrium when the banker is a price-taker in the loan market with both the private equilibrium when the banker internalizes the loan demand schedule and the socially optimal allocations. Comparing the two private equilibria, the banker extends more loans to entrepreneurs, which reduces the loan rate and the profit margin, when she does not internalize how her choice affects the loan demand by entrepreneurs. Naturally, the banker is worse off compared with the private equilibrium where she fully internalizes her actions. Entrepreneurs and savers are better off; the former because they get more loans and the latter because the banker raises more deposits to fund lending, which pushes up the deposit rate. It may seem that social welfare is higher in the private equilibrium with price-taking behavior compared with the socially optimal outcomes. This is not true. Consider, for example, that the social welfare weights are $w_{E}=w_{S}=0.1$. The difference between social welfare in the planner's solution and the private equilibrium with risktaking behavior is $1.06 \%$, i.e., the planner does better. The planner still cares about the banker and, thus, she chooses allocations that favor the banker, who did not internalize how her actions affected loan demand. The concerns about the banker's welfare diminish as more weight is placed on $S$ and $E$, which can be seen from the last column where the social welfare weights are $w_{E}=w_{S}=0.5$. Social welfare is higher by $0.05 \%$ in the planner solution compared with the private equilibrium with price-taking behavior. 


\begin{tabular}{|c|c|c|c|c|c|c|}
\hline & \multirow[t]{2}{*}{$\mathrm{PE}$} & \multicolumn{4}{|c|}{ SP for weights $\left(w_{E}, w_{S}\right)$} & \multirow[b]{2}{*}{$(0.50,0.50)$} \\
\hline & & Price-taking & $(0.00,0.20)$ & $(0.10,0.10)$ & $(0.20,0.00)$ & \\
\hline$I$ & 0.862 & 0.976 & 0.785 & 0.873 & 0.906 & 0.944 \\
\hline$L$ & 0.052 & 0.000 & 0.221 & 0.060 & 0.000 & 0.056 \\
\hline$D$ & 0.875 & 0.933 & 0.962 & 0.894 & 0.867 & 0.957 \\
\hline E & 0.038 & 0.043 & 0.044 & 0.039 & 0.038 & 0.043 \\
\hline$R_{I}$ & 4.097 & 3.966 & 4.198 & 4.089 & 4.042 & 4.015 \\
\hline$R_{D}$ & 1.717 & 1.981 & 1.804 & 1.767 & 1.758 & 1.996 \\
\hline$q$ & 0.407 & 0.400 & 0.386 & 0.403 & 0.408 & 0.395 \\
\hline$\ell$ & 0.057 & 0.000 & 0.219 & 0.065 & 0.000 & 0.056 \\
\hline$k$ & 0.042 & 0.044 & 0.044 & 0.042 & 0.042 & 0.043 \\
\hline$R_{I}-(1-\delta) R_{D}$ & 3.239 & 2.975 & 3.296 & 3.205 & 3.163 & 3.016 \\
\hline$I+L$ & 0.914 & 0.976 & 1.006 & 0.933 & 0.906 & 1.000 \\
\hline$I-E$ & 0.824 & 0.933 & 0.741 & 0.834 & 0.867 & 0.901 \\
\hline$E(D i v)$ & 0.745 & 0.740 & 0.755 & 0.747 & 0.743 & 0.739 \\
\hline$\Delta \mathbb{U}_{E}$ & - & $3.57 \%$ & $-1.66 \%$ & $0.33 \%$ & $1.19 \%$ & $2.57 \%$ \\
\hline$\Delta \mathbb{U}_{S}$ & - & $2.33 \%$ & $3.63 \%$ & $0.71 \%$ & $-0.30 \%$ & $3.37 \%$ \\
\hline$\Delta \mathbb{U}_{B}$ & - & $-1.60 \%$ & $-0.44 \%$ & $-0.05 \%$ & $-0.09 \%$ & $-1.57 \%$ \\
\hline
\end{tabular}

Table D.3: Private equilibrium allocations with and without price-taking banker's behavior versus Socially optimal solutions. The welfare changes are computed over the level of welfare in the private equilibrium where the banker internalizes the loan demand schedule, which is normalized to one for each agent.

\section{D.4 Perfectly Elastic Demand Curve}

This section studies the special case of a perfectly elastic demand curve, which obtains for $c_{I}=0$. Then, from (7), $R_{I}=A$, and, from (8), $\mathbb{U}_{E}^{*}=0$. There are two implications of abstracting from a downward sloping demand curve. First, the planner does not consider the welfare of entrepreneurs as they make zero profits. Second, the banker cannot manipulate her profit margin by adjusting the volume of lending to affect the loan rate and all of the adjustment in the loan-deposit spread is happening via the deposit rate.

Table D.4 reports the results from implementing single and combined regulatory tools, which are consistent with the results in the baseline model when the planner places more weight on savers. Tightening the leverage requirement forces the banker to raise more equity and results in some substitution away from liquid assets toward loans. The difference with the case of a downward sloping loan demand curve is that here the banker can extend more lending without pushing the loan rate down and, hence, eroding her profit margin. As in the general case, funding more loans with equity reduces the need for deposits, which also pushes down the deposit rate improving the margin and enabling the banker to raise more equity. Expected profits are higher, but the banker is worse off, because she had to contribute more equity than what was optimal for her in the private equilibrium.

The results for liquidity regulation are similar to the baselines ones. The only difference is that 
the reduction in lending does not boost loan rates, and hence the banker cannot increase deposittaking as much as she would be able to under a downward sloping demand curve. Contrary to the baseline case, the deposit rate falls because the lower run probability dominates the effect from the somewhat higher deposit demand. In the baseline case, lower lending could support a higher spread and, thus, the banker could increase deposit-taking a lot, undoing the effect of lower run-risk on deposit rates and pushing them to levels above their private equilibrium ones.

Comparing column " $\ell$ " and " $k \& \ell$ ", we see that leverage and liquidity regulations can be combined to improve welfare. The margin effect of adding a leverage requirement on top of the liquidity requirement is small in this example but goes in the direction of the baseline results. Finally, a subsidy on deposits, $\tau_{D}=-3.61 \%$, is needed to fully implement the planner's allocations, as is the case in the baseline model when the planner puts higher weight on savers.

\begin{tabular}{|c|c|c|c|c|c|}
\hline & $\mathrm{PE}$ & $k$ & $\ell$ & $k \& \ell$ & $k, \ell \& \tau_{D}$ \\
\hline$I$ & 0.550 & 0.558 & 0.503 & 0.503 & 0.510 \\
\hline$L$ & 0.109 & 0.103 & 0.193 & 0.193 & 0.195 \\
\hline$D$ & 0.627 & 0.625 & 0.663 & 0.663 & 0.672 \\
\hline E & 0.031 & 0.036 & 0.033 & 0.033 & 0.034 \\
\hline$R_{I}$ & 4.500 & 4.500 & 4.500 & 4.500 & 4.500 \\
\hline$R_{D}$ & 1.754 & 1.734 & 1.690 & 1.689 & 1.721 \\
\hline$q$ & 0.510 & 0.506 & 0.504 & 0.503 & 0.501 \\
\hline$\ell$ & 0.165 & 0.156 & 0.277 & 0.277 & 0.277 \\
\hline$k$ & 0.047 & 0.055 & 0.047 & 0.048 & 0.048 \\
\hline$R_{I}-(1-\delta) R_{D}$ & 2.423 & 2.433 & 2.455 & 2.455 & 2.439 \\
\hline$I+L$ & 0.658 & 0.661 & 0.695 & 0.696 & 0.706 \\
\hline$I-E$ & 0.519 & 0.522 & 0.470 & 0.470 & 0.477 \\
\hline$E(D i v)$ & 0.244 & 0.256 & 0.248 & 0.249 & 0.249 \\
\hline$\Delta \mathbb{U}_{E}$ & - & $0.00 \%$ & $0.00 \%$ & $0.00 \%$ & $0.00 \%$ \\
\hline$\Delta \mathbb{U}_{S}$ & - & $-0.05 \%$ & $0.962 \%$ & $0.965 \%$ & $1.23 \%$ \\
\hline$\Delta \mathbb{U}_{B}$ & - & $-0.05 \%$ & $-0.06 \%$ & $-0.06 \%$ & $-0.08 \%$ \\
\hline$\Delta \mathbb{S}_{s p}$ & - & $-0.01 \%$ & $0.90 \%$ & $0.90 \%$ & $1.16 \%$ \\
\hline
\end{tabular}

Table D.4: Equilibrium allocation under perfectly elastic loan demand. The welfare changes are computed over the level of welfare in the private equilibrium, which is normalized to one for each agent.

\section{D.5 Direct Lending}

This section derives the conditions for direct lending to entrepreneurs by savers and computes the equilibrium outcomes for the parametrization in section 3.2.

Direct lending requires the individual savers to be able to monitor the entrepreneur. Denote by $X_{S}$ the monitoring cost to an individual saver, which we assume can be higher or equal to the monitoring cost of the banker, i.e., $X_{S} \geq X$. At $t=1$, an individual saver can lend to the entrepreneur, 
$I_{d l}$, at interest rate $r_{d l}$. In the intermediate period, she would liquidate all of her loans if she turns out to be impatient. Otherwise, the saver waits until the final period and receives the percentage repayment on the loans she made. The saver's utility under direct lending is given by

$$
\mathbb{U}_{S}^{d l}=U\left(e_{R}-I_{d l}\right)+\beta \delta \int_{\underline{\xi}}^{\bar{\xi}} \xi I_{d l} \frac{d \xi}{\Delta_{\xi}}+\beta^{2}(1-\delta) \sum_{s}\left(\omega I_{d l}\left(1+r_{d l}\right)-X_{S}\right) \frac{d \xi}{\Delta_{\xi}} .
$$

The entrepreneur will choose $I_{d l}$ to maximize her utility $\mathbb{U}_{E}^{d l}=(1-\delta)\left[\omega I_{d l}\left(1+r_{d l}\right)-c\left(I_{d l}\right)\right]$ ; with probability $\delta$, an individual entrepreneur has her project liquidated and receives zero utility, while, with probability $1-\delta$, the saver does not liquidate the project, and the entrepreneur incurs the effort cost and defaults in the bad state. E's optimizing behavior yields the following loan demand schedule:

$$
1+r_{d l}=A-c^{\prime}\left(I_{d l}\right) / \omega
$$

Because each individual saver is sufficiently small, she takes the loan rate as given and, thus, the loan supply schedule is:

$$
1+r_{d l}=\frac{1}{\omega \beta^{2}(1-\delta)}\left[U^{\prime}\left(e_{R}-I_{d l}\right)-\beta \delta E(\xi)\right]
$$

The intersection of the loan demand and loan supply schedule in (D.7) and (D.8) yields the equilibrium loan rate and loan amount. Finally, $S$ 's and $E$ 's levels of welfare in equilibrium are given by $\mathbb{U}_{S}^{d l *}=U\left(e_{R}-I_{d l}\right)+U^{\prime}\left(e_{R}-I_{d l}\right) I_{d l}-\beta^{2}(1-\delta) X_{S}$ and $\mathbb{U}_{E}^{d l *}=(1-\delta)\left(c^{\prime}\left(I_{d l}\right) I_{d l}-c\left(I_{d} l\right)\right)$. Table D.5 reports the equilibrium in the loan market together with how $S$ 's and $E$ 's levels of welfare compare across three cases: the banking private equilibrium reported in section 3.2, the direct lending equilibrium, and the autarkic outcome when $S$ uses only the storage technology. Savers are better off under bank intermediation as they enjoy the transaction services of deposits, and they do not need to pay the monitoring cost. However, for $X=X_{S}$, savers are better off lending directly to $E$ compared with autarky. There is a level of the monitoring $\operatorname{cost} X_{S}$ that this stops being true $\left(X_{S} / X>1.46\right.$ in our example). Direct lending is higher than bank lending because the bank strategically curtails credit extension to secure higher loan rates and increase her profit margins. Note that the monitoring cost does not affect the marginal choice of $I_{d l}$ because it is not incurred per unit of loans extended but rather applies to the whole portfolio.

\begin{tabular}{lrrrr}
\hline \hline & Loan rate & Loan amount & $\% \Delta \mathbb{U}_{S}$ & $\% \Delta \mathbb{U}_{E}$ \\
\hline Intermediation & 4.097 & 0.862 & - & - \\
Direct lending & 2.272 & 0.928 & $-3.20 \%$ & $0.38 \%$ \\
Autarky & - & - & $-4.34 \%$ & $-7.61 \%$ \\
\hline \hline
\end{tabular}

Table D.5: Equilibrium allocation under bank intermediation, direct lending, and autarky with storage. The welfare changes are computed over the level of welfare in the private equilibrium with bank intermediation, which is normalized to one for each agent. 


\section{D.6 Negative Interest Rates and Run-Proof Banking}

In our benchmark results in section 3.2, we assumed that the bank cannot set (charge) negative net interest rates for early withdrawals denoted by $\hat{r}_{D}=r_{D}-1$, i.e., $r_{D} \geq 1$. In this section, we relax this assumption. On the one hand, a negative $\hat{r}_{D}$ may allow the bank to eliminate all run-risk, i.e., the bank is run-proof despite issuing demandable deposits. On the other hand, a negative $\hat{r}_{D}$ would reduce the utility from transaction services and the bank would need to offer a higher late deposit rate, $R_{D}$; otherwise, savers may choose to self-insure by holding the liquid asset and stop using the bank.

There are two subtle assumptions we have made that offer the best chance for negative rates to eliminate run-risk without hurting welfare. The first assumption is that $V(0)=0$, i.e., if the bank sets $r_{D}=0$, then savers get zero utility. This is important because, if $V(0) \rightarrow-\infty$, savers would become explosively worse off as $\hat{r}_{D}$ became more and more negative, and they would require an explosively high $R_{D}$ to supply deposits. As a result, intermediation would be impossible under very negative $\hat{r}_{D}$. The second assumption is that the liquid asset cannot offer the transaction services of deposits (or if it does, its services are inferior to the ones offered by deposits). If deposits and the liquid assets were perfect substitute for transactions, then savers would very quickly switch to holding the liquid assets once $\hat{r}_{D}$ became sufficiently negative. Instead, in our environment, savers would still be willing to hold deposits along with the liquid asset, even under considerably negative $\hat{r}_{D}$. The reason is that savers' utility under bank intermediation is strictly higher than in autarky for $r_{D}>0$ (see the discussion at the end of section 2.1).

We derive below the conditions under which run-proof banking is possible as well as the corresponding private and social equilibrium allocation for our benchmark parametrization. Our analysis has focused on the consequences induced by the banker's behavior when there is positive run-risk in equilibrium. Thus, the natural candidate for comparison is run-proof banking, which is possible given the two aforementioned assumptions. ${ }^{5}$

Banking is run-proof if $\hat{\lambda}(\underline{\xi})$ is not lower than one. Hence, liquid asset holdings should satisfy:

$$
\begin{aligned}
\hat{\lambda}(\underline{\xi}) & =\frac{\theta(\underline{\xi}, 1)-\frac{\underline{\xi} R_{D}}{r_{D} R_{I}}-\frac{\xi X / \omega}{D r_{D} R_{I}}}{1-\frac{\underline{\xi} R_{D}}{r_{D} R_{I}}} \geq 1 \Rightarrow \theta(\underline{\xi}, 1) \geq 1+\frac{\underline{\xi} X / \omega}{D r_{D} R_{I}} \\
& \Rightarrow L \geq D r_{D}-\underline{\xi}\left(I-\frac{X}{\omega R_{I}}\right) .
\end{aligned}
$$

Beyond that level, it is inefficient to hold liquid assets, so (D.9) holds with equality.

If $L \geq \delta D r_{D}$ or

$$
(1-\delta) D r_{D} \geq \underline{\xi}\left(I-\frac{X}{\omega R_{I}}\right)
$$

\footnotetext{
${ }^{5}$ This does not mean that negative interest rates are only compatible with run-proof banking. Although quantitatively the results in section 3.2 will differ if we allow for negative $\hat{r}_{D}$, the wedges derived in section 3.1 will maintain their functional form and, hence, the sources of divergence between the privately and socially optimal solutions will be the same.
} 
then the liquid asset holdings of the bank are higher than the predictable early withdrawals and the bank will transfer the excess liquidity, $\widehat{L}=(1-\delta) D r_{D}-\xi\left(I-X /\left(\omega R_{I}\right)\right)$ in the last period. Otherwise, the bank will need to recall a fraction $\hat{y}(\xi)=\left[\xi\left(I-X /\left(\omega R_{I}\right)\right)-(1-\delta) D r_{D}\right] /(\xi I)$ of loans to pay early withdrawals. Note that, if $\underline{\xi}$ is very small, then (D.10) is satisfied for a larger range of negative $\hat{r}_{D}$; at the limit, as $\underline{\xi} \rightarrow 0$, (D.10) is satisfied for all $r_{D}>0$, i.e., the bank does not need to recall any loans. For the rest of this section, we assume that $\underline{\xi} \rightarrow 0$ in order to simplify the algebra.

In the absence of run-risk, the loan demand schedule can be written as

$$
R_{I}=A-c^{\prime}(I) / \omega
$$

The deposit supply schedule is more elaborate, because the excess liquidity $\widehat{L}$ will be distributed pro-rata to the $(1-\delta)$ patient savers when the bank defaults in the bad state of the world. ${ }^{6}$ Thus, the percentage payment, $\mathcal{R}$, to patient savers in the bad state is $\mathcal{R}=\widehat{L} /\left((1-\delta) D R_{D}\right)$ and the total payoff from holding deposits in the bad state is $\mathcal{R} D R_{D}$. Individual savers take the percentage payment in the bad state as given when choosing the amount of deposits. The deposit supply schedule is, thus, given by the following first-order condition with respect to $D$ :

$$
\begin{aligned}
& \overbrace{-U^{\prime}\left(e_{S}-D-L_{S}\right)}^{\begin{array}{c}
\text { Consumption cost } \\
\text { of depositing }
\end{array}}+\overbrace{\beta \delta r_{D}}^{\begin{array}{c}
\text { Marginal payoff to } \\
\text { impatient } S
\end{array}}+\overbrace{\beta^{2}(1-\delta) \omega R_{D}}^{\begin{array}{c}
\text { Marginal payoff to } \\
\text { patient } S \text { in good state }
\end{array}}+\overbrace{\beta^{2}(1-\delta)(1-\omega) \mathcal{R} R_{D}}^{\begin{array}{c}
\text { Marginal payoff to } \\
\text { patient } S \text { in bad state }
\end{array}} \\
& +\underbrace{V^{\prime}\left(D r_{D}\right) r_{D}}_{\begin{array}{c}
\text { Marginal payoff from } \\
\text { transaction services }
\end{array}}=0 \\
& \Rightarrow \omega(1-\delta)\left(R_{D}-r_{D}\right)=\frac{U^{\prime}\left(e_{S}-D-L_{S}\right)-V^{\prime}\left(D r_{D}\right) r_{D}-\left(\beta \delta+\beta^{2}(1-\delta)\right) r_{D}}{\beta^{2}},
\end{aligned}
$$

where we have substituted the definition of $\mathcal{R}$.

A negative $\hat{r}_{D}$ makes self-insuring through holding the liquid asset more appealing to savers. As a result, $L_{S}$ can be positive, in contrast to the benchmark equilibrium we have studied, and (3) will hold with equality. We assume that this is the case and verify our conjecture in equilibrium, under the same parametrization used for the benchmark equilibrium. Hence,

$$
U^{\prime}\left(e_{S}-D-L_{S}\right)=\beta \delta+\beta^{2}(1-\delta)
$$

Using the balance sheet constraint (9) and (D.9), we can express equity in terms of the lending, deposit and early deposit rate choices:

$$
E=I+D r_{D}
$$

\footnotetext{
${ }^{6}$ In the bad state, the bank defaults if $\widehat{L}<(1-\delta) D R_{D}$, which is true because $R_{D}>r_{D}$.
} 
Moreover, using (D.9), (D.11), (D.12), (D.13), and (D.14), the utility of the banker can be rewritten as:

$$
\begin{aligned}
\mathbb{U}_{B}^{n r}=W\left(e_{B}-E\right)+\omega\left[R_{I} I+\widehat{L}-\right. & \left.(1-\delta) D R_{D}\right]-X \\
=W\left(e_{B}-I-D r_{D}\right)+\omega[ & A I-c^{\prime}(I) I / \omega+\beta^{-2} V^{\prime}\left(D r_{D}\right) r_{D} \\
& \left.+\left(\beta^{-1} \delta+(1-\delta)\right) r_{D}\right]-X .
\end{aligned}
$$

The private equilibrium is characterized by the choice of $I, D$, and $r_{D}$ that result in the highest $\mathbb{U}_{B}^{n r}$ in (D.15), i.e., by the following first-order condition:

$$
\begin{aligned}
& -W^{\prime}\left(e_{B}-I-D r_{D}\right)+\omega A-c^{\prime \prime}(I) I-c^{\prime}(I)=0, \\
& -W^{\prime}\left(e_{B}-I-D r_{D}\right) r_{D}+\omega \beta^{-2} V^{\prime \prime}\left(D r_{D}\right) r_{D}^{2}=0,
\end{aligned}
$$

and

$$
-W^{\prime}\left(e_{B}-I-D r_{D}\right) D+\omega \beta^{-2} V^{\prime \prime}\left(D r_{D}\right) D r_{D}+\omega\left(\beta^{-1} \delta+(1-\delta)\right)=0 .
$$

Table D.6 reports the equilibrium outcomes such that the bank is run-proof. In order to satisfy (D.9), the banker has an incentive to set a negative net deposit rate for early withdrawals; otherwise, she would need to hold as many liquid asset as the amount of deposits and could not use any to extend loans. Eliminating the run reduces the risk premium savers demand to hold deposits, but it also reduces the transaction services to savers and hence the convenience yield that the bank extracts. In particular, there is a trade-off between $r_{D}$ and $R_{D}$. Given that deposits are safer, savers are willing to accept a late deposit rate $R_{D}$ less than one in exchange for a less negative $\hat{r}_{D}$. A more negative $\hat{r}_{D}$ allows the banker to channel more deposits to loans and still eliminate all run-risk. But, savers would demand higher compensation in terms of $R_{D}$, which reduces banking profits. The banker balances these two effects and offers deposit rates that are making savers indifferent between supplying deposits and self-insuring ( $L_{S}>0$ in the run-proof equilibrium). Overall, lending is lower in the run-proof private equilibrium, while the liquid asset holdings are substantially higher compared to our benchmark PE where we restrict $r_{D} \geq 1$. Still, the level deposits are comparable across the two private equilibria. For our parametrization, all agents are worse off in the run-proof equilibrium. Nevertheless, this does not always need to be always the case. For example, if $\underline{\xi}$ was high enough, then the bank could be run-proof without the need to hold a lot of liquid assets, which hurt lending, or charge negative deposit rates for early withdrawals, which diminish transaction services.

Substituting (D.11), (D.12), and (D.13) in (5) and (1), and setting $q=0$, we get the following indirect utility functions:

$$
\mathbb{U}_{E}^{n r, *}=c^{\prime}(I) I-c(I)
$$

and

$$
\mathbb{U}_{S}^{n r, *}=\mathbb{U}_{S}^{\alpha}+V\left(D r_{D}\right)-V^{\prime}\left(D r_{D}\right) D r_{D}
$$




\begin{tabular}{|c|c|c|c|c|c|}
\hline & \multirow{2}{*}{$\begin{array}{r}\mathrm{PE} \\
\text { Restrict } r_{D} \geq 1\end{array}$} & \multirow{2}{*}{$\begin{array}{r}\mathrm{PE} \\
\text { Run-proof }\end{array}$} & \multicolumn{3}{|c|}{ Run-proof SP for weights $\left(w_{E}, w_{S}\right)$} \\
\hline & & & $(0.05,0.15)$ & $(0.10,0.10)$ & $(0.15,0.05)$ \\
\hline$I$ & 0.853 & 0.114 & 0.104 & 0.107 & 0.111 \\
\hline$L$ & 0.061 & 0.683 & 0.693 & 0.689 & 0.686 \\
\hline$D$ & 0.876 & 0.739 & 0.739 & 0.739 & 0.739 \\
\hline E & 0.039 & 0.058 & 0.058 & 0.058 & 0.058 \\
\hline$R_{I}$ & 4.108 & 4.495 & 4.496 & 4.496 & 4.495 \\
\hline$r_{D}$ & 1.000 & 0.924 & 0.938 & 0.933 & 0.929 \\
\hline$R_{D}$ & 1.726 & 0.666 & 0.666 & 0.660 & 0.663 \\
\hline$q$ & 0.408 & 0.000 & 0.000 & 0.000 & 0.000 \\
\hline$\ell$ & 0.067 & 0.857 & 0.869 & 0.865 & 0.861 \\
\hline$k$ & 0.042 & 0.073 & 0.073 & 0.073 & 0.073 \\
\hline$R_{I}-(1-\delta) R_{D}$ & 3.245 & 4.162 & 4.163 & 4.166 & 4.164 \\
\hline$I+L$ & 0.915 & 0.797 & 0.797 & 0.797 & 0.797 \\
\hline$I-E$ & 0.815 & 0.056 & 0.046 & 0.049 & 0.053 \\
\hline$L_{S}$ & 0.000 & 0.081 & 0.080 & 0.081 & 0.081 \\
\hline$\Delta \mathbb{U}_{E}$ & - & $-7.33 \%$ & $-7.33 \%$ & $-7.33 \%$ & $-7.33 \%$ \\
\hline$\Delta \mathbb{U}_{S}$ & - & $-1.26 \%$ & $-1.19 \%$ & $-1.21 \%$ & $-1.23 \%$ \\
\hline$\Delta \mathbb{U}_{B}$ & - & $-40.94 \%$ & $-43.81 \%$ & $-42.85 \%$ & $-41.90 \%$ \\
\hline
\end{tabular}

Table D.6: Private equilibrium and socially optimal run-proof allocations. The welfare changes are computed over the level of welfare in the private equilibrium where deposit rates are restricted to be positive and banking is not run-proof. All equilibria are for $\underline{\xi}=0$.

The run-proof socially optimal equilibrium allocation maximize a social welfare function $\mathbb{U}_{s p}^{n r}=$ $\mathbb{U}_{B}^{n r}+w_{E} \mathbb{U}_{E}^{n r, *}+w_{S} \mathbb{U}_{S}^{n r, *}$ given (D.15), (D.19), and (D.20). As a result, the first-order conditions characterizing the planner's solution are

$$
\begin{aligned}
& -W^{\prime}\left(e_{B}-I-D r_{D}\right)+\omega A-c^{\prime \prime}(I) I-c^{\prime}(I)+w_{E} \partial \mathbb{U}_{E}^{n r * *} / \partial I=0, \\
& -W^{\prime}\left(e_{B}-I-D r_{D}\right) r_{D}+\omega \beta^{-2} V^{\prime \prime}\left(D r_{D}\right) r_{D}^{2}+w_{S} \partial \mathbb{U}_{S}^{n r, *} / \partial D=0,
\end{aligned}
$$

and

$$
-W^{\prime}\left(e_{B}-I-D r_{D}\right) D+\omega \beta^{-2} V^{\prime \prime}\left(D r_{D}\right) D r_{D}+\omega\left(\beta^{-1} \delta+(1-\delta)\right)+w_{S} \partial \mathbb{U}_{S}^{n r, *} / \partial r_{D}=0,
$$

where $\partial \mathbb{U}_{E}^{n r, *} / \partial I=c^{\prime \prime}(I) I>0, \partial \mathbb{U}_{S}^{n r, *} / \partial D=-V^{\prime \prime}\left(D r_{D}\right) D r_{D}^{2}>0$, and $\partial \mathbb{U}_{S}^{n r, *} / \partial r_{D}=-V^{\prime \prime}\left(D r_{D}\right) D^{2} r_{D}>$ 0 .

When the planner puts more weight on the saver, she choose a less negative $r_{D}$ and, hence, needs higher $L$ to implement the run-proof equilibrium, which pushes lending down compared with the private equilibrium or cases where less weight is put on $S$. Both the banker and entrepreneurs 
are worse off compared with the private equilibrium-the welfare loss for the entrepreneur is small because lending and investment are already low in the private run-proof equilibrium, and the higher investment is, the bigger the surplus to $E$ due to the convex effort cost. Overall, all agents are worse off, even for the planner's allocations, compared with the benchmark private equilibrium indicating that run-proof banking is not optimal for the example we present.

\section{D.7 Outside Equity}

This section extends the baseline model such that the bank has an alternative source of funding apart from the equity contributed by the banker and the deposits offered by savers. In particular, we consider a separate group of agents, who we call outside investors and who may choose to buy equity at a certain price from the bank at $t=1$ in exchange for a share of the profits at $t=3$. These investors do not have a preference for early consumption nor do they value the transaction services of deposits contrary to savers. We assume that their preferences are the same as for bankers, but contrary to them, investors do not have the ability to monitor entrepreneurs and, hence, manage a bank themselves. We will refer to the equity contributed by the banker and investors as inside equity and outside equity, respectively. ${ }^{7}$

Denote by $P$ the price of one share of outside equity and by $O$ the number of shares issued and distributed to outside investors. Inside equity is equally divided into $E$ shares, i.e., the banker first injects inside equity, normalizing the price of each (inside equity) share to one, and then decides how many shares to issue to outside investors and at what price. Thus, the total number of shares is $E+O$ and the total equity capital $E+P O$. It is convenient to denote bank profits as $\operatorname{Div}(\xi, \delta)=$ $\omega\left[(1-y(\xi, \delta)) I R_{I}-(1-\delta) D R_{D}\right]$ and profits per share as $\operatorname{DPS}(\xi, \delta)=\operatorname{Div}(\xi, \delta) /(E+O)$. Then, investors choose how much of their period 1 endowment, $e_{O}$, to invest in equity, in order to maximize

$$
\mathbb{U}_{O}=W\left(e_{O}-P O\right)+\int_{\xi^{*}}^{\bar{\xi}} O D P S(\xi, \delta) \frac{d \xi}{\Delta_{\xi}},
$$

which, taking the profits per share as given, yields the following outside equity supply (ES) schedule:

$$
-P W^{\prime}\left(e_{O}-P O\right)+\int_{\xi^{*}}^{\bar{\xi}} D P S(\xi, \delta) \frac{d \xi}{\Delta_{\xi}} \leq 0,
$$

holding with equality for $O>0$. Finally, substituting (D.25) in (D.24) we get the following indirect

\footnotetext{
${ }^{7}$ We have assumed a different investor base for outside equity to keep the extension simple. Note that outside equity and deposit markets can be endogenously segmented, i.e., there is no need to exogenously restrict outside investors or depositors to supply deposits or equity, respectively. The decision to abstain from these markets would be consistent with equilibrium equity prices and deposit rates. Intuitively, savers would require a lower price to purchase equity, because equity is less useful for early consumption, because it is worthless in a run and secondary market trading can be frictional, and because it does not provide transaction services. Moreover, an all-equity funding structure would not be possible even if the bank preferred it due to the disciplinary role of runnable debt. Similarly, outside investors would require a higher deposit rate to supply deposits, since they do not price their transaction services.
} 
utility for outside investors:

$$
\mathbb{U}_{O}^{*}=W\left(e_{O}-P O\right)+P O W^{\prime}\left(e_{O}-P O\right) .
$$

On top of the previous choices in $C$, the banker will also choose the level of outside equity, $O$, and the price, $P$, that are consistent with the equity supply schedule (D.25). Hence, $B$ 's choice set becomes $\widetilde{C}=C \cup\{O, P\}$. Because $B$ will receive only a fraction $E /(E+O)$ of the profits, her utility becomes:

$$
\begin{aligned}
\widetilde{\mathbb{U}}_{B} & =W\left(e_{B}-E\right)+\int_{\xi^{*}}^{\bar{\xi}} \operatorname{EDPS}(\xi, \delta) \frac{d \xi}{\Delta_{\xi}} \\
& =W\left(e_{B}-E\right)+\int_{\xi^{*}}^{\bar{\xi}}\left\{\frac{E}{E+O} \omega\left[(1-y(\xi, \delta)) I R_{I}-(1-\delta) D R_{D}\right]-X\right\} \frac{d \xi}{\Delta_{\xi}} .
\end{aligned}
$$

Note that the banker bears the full cost of monitoring.

The functional form of the deposit supply and loan demand schedules, (2) and (7) respectively, are unaffected by the introduction of outside equity. However, the monitoring threshold $\hat{\lambda}$, given by (??) for certain $\xi$, will change to $\hat{\tilde{\lambda}}$, because the banker will monitor if her share of, rather than the total, profits is higher than the monitoring cost:

$$
\begin{aligned}
& \frac{E}{E+O} \omega\left[(1-y(\xi, \hat{\tilde{\lambda}})) I R_{I}-(1-\hat{\tilde{\lambda}}) D R_{D}\right]-X \geq 0 \\
\Rightarrow & \hat{\tilde{\lambda}}(\xi)=\frac{(\xi I+L) R_{I}-\xi\left(D R_{D}+\frac{E+O}{E} \frac{X}{\omega}\right)}{D\left[r_{D} R_{I}-D\right.} .
\end{aligned}
$$

In other words, outside equity reduces the threshold for withdrawals under which the banker has incentives to monitor, i.e., $\partial \hat{\tilde{\lambda}} / \partial O<0$. The functional form of the global game constraint (32) does not change but $\lambda^{*}$ is replaced with $\tilde{\lambda}^{*} \equiv \hat{\tilde{\lambda}}\left(\xi^{*}\right)$ in the limits of integration:

$$
\widetilde{G G}=\int_{\delta}^{\tilde{\lambda}^{*}}\left[\omega D R_{D}-D r_{D}\right] d \lambda-\int_{\tilde{\lambda}^{*}}^{\theta^{*}} D r_{D} d \lambda-\int_{\theta^{*}}^{1} \frac{L+\xi^{*} I}{\lambda} d \lambda=0 .
$$

Finally, the balance sheet incorporates the funds form raising outside equity:

$$
\widetilde{B S}: \quad I+L=D+E+P O .
$$

$B$ chooses variables in $\widetilde{C}$ to maximize $\widetilde{\mathbb{U}}_{B}$ in (D.27) subject to $\widetilde{\mathcal{Y}}=\{\widetilde{B S}, \widetilde{G G}, D S, L D, E S\}$ given by (D.30), (D.29), (2), (7), and (D.25). The private equilibrium is characterized by the following first-order conditions:

$$
\frac{\partial \widetilde{\mathbb{U}}_{B}}{\partial \widetilde{C}}+\sum_{\widetilde{\mathcal{Y}}} \psi_{\widetilde{\gamma}} \frac{\partial \tilde{\mathcal{Y}}}{\partial \widetilde{C}}=0,
$$

where $\psi_{E S}$ is the Lagrange multiplier on the equity supply schedule and $\partial E S / \partial \widetilde{C}$ are the partial 
derivatives reported in (C.59)-(C.68) in section C. $3{ }^{8}$ Thus, the banker also internalizes how her choices affect the supply of outside equity by investors. At the same time, issuing outside equity has a direct effect on the disutility from injecting inside equity as can be seen by $\partial \widetilde{\mathbb{U}}_{B} / \partial E=\partial \mathbb{U}_{B} / \partial E+$ $O /(E+O) \int_{\xi^{*}}^{\bar{\xi}} D P S(\xi, \delta) d \xi / \Delta_{\xi}$, where $\partial \mathbb{U}_{B} / \partial E<0$ is given by (C.22). Hence, if the issuance of outside equity is positive, the disutility for the banker from injecting equity is lower because it increases her share of profits. We now turn to the choice of $O$ and $P$, which are new to our benchmark analysis.

The first-order conditions (D.31) with respect to $O$ and $P$ are:

$$
\frac{\partial \widetilde{\mathbb{U}}_{B}}{\partial O}+\psi_{B S} P+\psi_{G G} \frac{\partial \widetilde{G G}}{\partial O}+\psi_{E S} \frac{\partial E S}{\partial O}=0
$$

and

$$
\psi_{B S} O+\psi_{E S} \frac{\partial E S}{\partial P}=0
$$

Substituting (D.33) in (D.32) we get:

$$
\underbrace{-\left(\frac{\partial \widetilde{\mathbb{U}}_{B}}{\partial O}+\psi_{G G} \frac{\partial \widetilde{G G}}{\partial O}\right)}_{\begin{array}{c}
\text { Cost of issuing } \\
\text { outside equity }
\end{array}}=\underbrace{\psi_{B S}\left(P-O \frac{\partial E S^{-1}}{\partial P} \frac{\partial E S}{\partial O}\right)}_{\begin{array}{c}
\text { Benefit of issuing } \\
\text { outside equity }
\end{array}},
$$

where $\partial \widetilde{\mathbb{U}}_{B} / \partial O=-E /(E+O) \int_{\xi^{*}}^{\bar{\xi}} D P S(\xi, \delta) d \xi / \Delta_{\xi}<0, \partial E S / \partial O<0$ from (C.63), $\partial E S / \partial P<0$ from (C.68), and $\partial \widetilde{G G} / \partial O=\omega D R_{D} \partial \hat{\tilde{\lambda}} / \partial O<0$, because $\partial \hat{\tilde{\lambda}} / \partial O=-\xi / E X / \omega /\left\{D\left[r_{D} R_{I}-{ }_{D}\right]\right\}<0$.

The cost of issuing outside equity consists of two components. First, outside equity reduces the share of profits accruing to the banker, and second, it makes monitoring less likely, which adversely affects the probability of a run, all else being equal. Note that this does not mean that issuing outside equity increases the run probability in equilibrium, since other variables will adjust and the bank may operate with more capital and liquidity reducing the run probability, as we show in the numerical results below. The benefit of issuing outside equity stems from raising additional funds given the shadow value of funding $\psi_{B S}$. The banker does not take $P$ as given, but internalizes how her choice of $O$ affects $P$ via $E S$ and, hence, accurately captures the marginal funding benefit from issuing outside equity.

The social planner faces the same choices $\widetilde{C}$ and constraints $\widetilde{Y}$ as the banker in the private equilibrium but wants to maximize the social welfare function $\widetilde{\mathbb{U}}_{s p}^{*}=\widetilde{\mathbb{U}}_{B}+w_{S} \mathbb{U}_{S}^{*}+w_{E} \mathbb{U}_{E}^{*}+w_{S} \widetilde{\mathbb{U}}_{O}^{*}$,

\footnotetext{
${ }^{8}$ The partial derivatives $\partial D S / \partial C$ and $\partial L D / \partial C$ are given by (C.43)-(C.50) and (C.51)-(C.58), respectively, while all $\partial D S / \partial O, \partial D S / \partial P, \partial L D / \partial O$, and $\partial D S / \partial P$ are zero. For choices $c^{\prime}=\left\{I, L, D, R_{I}, r_{D}, R_{D}\right\}, \partial \widetilde{\mathbb{U}}_{B} / \partial c^{\prime}=E /(E+O) \partial \mathbb{U}_{B} / \partial c^{\prime}$, where $\partial \mathbb{U}_{B} / \partial c^{\prime}$ are given by (C.19), (C.20), (C.21), (C.24), (C.25) and (C.26), while $\partial \widetilde{\mathbb{U}}_{B} / \partial \xi^{*}=\partial \mathbb{U}_{B} / \partial \xi^{*}+O D P S\left(\xi^{*}, \delta\right)$ using (C.23). The partial derivatives $\partial \widetilde{G G} / \partial C$ have the same functional form given by (C.35)-(C.42) with the exception that $\partial \hat{\lambda} / \partial C$ is replaced by $\partial \hat{\tilde{\lambda}} / \partial C$. In turn, the latter partial derivatives have the same function form with the former given by (C.27)-(C.29) and (C.32)-(C.34) with the exception of the partial derivatives with respect to $E$ and $\xi^{*}$, which need to account for outside equity. That is, $\partial \hat{\tilde{\lambda}} / \partial E=\xi O / E^{2} X / \omega /\left\{D\left[r_{D} R_{I}-D\right]\right\}$ and $\partial \hat{\tilde{\lambda}} / \partial \xi^{*}=\partial \hat{\lambda} / \partial \xi^{*}-O / E X / \omega /\left\{D\left[r_{D} R_{I}-D_{D}\right]\right\}$ using (C.31). The remaining partial derivatives are discussed in the main text.
} 
where the utilities are defined in (D.27), (4), (8), (D.26), and $w_{O} \geq 0$ is the weight assigned to outside investors.

Table D.7 reports the private and social equilibrium outcomes when the bank issues outside equity and the planner assigns zero weight to outside investors, such that we can have a more straightforward comparison to the case that there is no outside equity funding. We, first, compare the private equilibrium outcomes. Issuing outside equity allows the banker to expand the balance sheet, but reduces her share of profits. To compensate for this, the banker decreases lending to improve the profit margin and also injects more inside equity. Relying more on equity funding allows the banker to channel more deposits into the liquid asset resulting in fewer loans funded by deposits. The higher liquidity and capital ratios outweigh the negative effect of outside equity on monitoring incentives and result in lower run-risk compared to the PE without issuance of outside equity. Deposits become safer and the bank can attract deposits offering lower deposit rates, which further improves the profit margin. Although it is not reported in the table, the banker enjoys higher utility in the PE where she issues outside equity compared with the benchmark equilibrium. Comparing the privately and socially optimal outcomes when the bank issues outside equity, we derive the same conclusion as in the benchmark case. The planner chooses more liquidity and capital to favor $S$ resulting in fewer loans funded by deposits, but also lower run-risk. On the contrary, the planner cuts the liquid asset holdings to support more lending and favor $E$ resulting in higher run-risk compared with the private equilibrium. ${ }^{9}$ Finally, comparing the socially optimal outcomes with and without the issuance of outside equity, we find that the planner can implement lower run-risk and achieve higher social welfare when outside equity funding is allowed.

We should note that these observations do not rely on the fact that the planner assigns zero weight on outside investors. If $w_{O}>0$, the planner internalizes how the issuance of outside equity matters for outside investors' welfare via (D.26). Hence, the first-order conditions with respect to $O$ and $P$-given by (D.32) and (D.32) in PE and SP for $w_{O}=0$-incorporate additional terms:

$$
\frac{\partial \widetilde{\mathbb{U}}_{B}}{\partial O}+\psi_{B S} P+\psi_{G G} \frac{\partial \widetilde{G G}}{\partial O}+\psi_{E S} \frac{\partial E S}{\partial O}+w_{O} \frac{\partial \mathbb{U}_{O}^{*}}{\partial O}=0
$$

and

$$
\psi_{B S} O+\psi_{E S} \frac{\partial E S}{\partial P}+w_{O} \frac{\partial \mathbb{U}_{O}^{*}}{\partial P}=0
$$

where $\partial \mathbb{U}_{O}^{*} / \partial O=-P^{2} O W^{\prime \prime}\left(e_{O}-P O\right)>0$ and $\partial \mathbb{U}_{O}^{*} / \partial P=-P O^{2} W^{\prime \prime}\left(e_{O}-P O\right)>0$.

Table D.8 reports the privately and socially optimal outcomes when the planner assigns $w_{O}>0$. In sum, the planner offers a better price $P$ to outside investors compared with the case that $w_{O}=0$, whose welfare improves. But, the rest of the findings remain unchanged.

\footnotetext{
${ }^{9}$ One inconsequential difference is that for $\left(w_{E}, w_{S}\right)=(0.1,0.1)$ the planner chooses lower lending compared with the private equilibrium in the presence of outside equity funding, which results in lower utility for $E$. The reason is that issuing outside equity can help savers further, and, as we show, social welfare is higher for this set of weights. If, instead, we set $\left(w_{E}, w_{S}\right)=(0.12,0.08)$, we find that lending as well as liquidity and capital are higher resulting in higher welfare for both $E$ and $S$ compared with the private equilibrium (as is the case in absence of outside equity funding for $\left.\left(w_{E}, w_{S}\right)=(0.1,0.1)\right)$.
} 


\begin{tabular}{|c|c|c|c|c|c|c|}
\hline & \multirow{2}{*}{\multicolumn{2}{|c|}{ 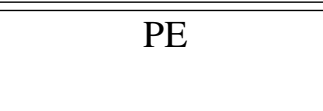 }} & \multicolumn{4}{|c|}{ SP for weights $\left(w_{E}, w_{S}\right)$} \\
\hline & & & \multicolumn{2}{|c|}{$(0.1,0.1)$} & \multicolumn{2}{|c|}{$(0.2,0.0)$} \\
\hline & No $O E$ & $O E$ & No $O E$ & $O E$ & No $O E$ & $O E$ \\
\hline$I$ & 0.862 & 0.834 & 0.873 & 0.829 & 0.906 & 0.908 \\
\hline$L_{1}$ & 0.052 & 0.131 & 0.060 & 0.168 & 0.000 & 0.020 \\
\hline$D$ & 0.875 & 0.908 & 0.894 & 0.937 & 0.867 & 0.875 \\
\hline E & 0.038 & 0.041 & 0.039 & 0.043 & 0.038 & 0.039 \\
\hline$O$ & 0.000 & 0.002 & 0.000 & 0.002 & 0.000 & 0.001 \\
\hline$E /(E+O)$ & 1.000 & 0.962 & 1.000 & 0.959 & 1.000 & 0.965 \\
\hline$R_{I}$ & 4.097 & 4.142 & 4.089 & 4.154 & 4.042 & 4.047 \\
\hline$R_{D}$ & 1.717 & 1.697 & 1.767 & 1.748 & 1.758 & 1.727 \\
\hline$P$ & - & 9.902 & - & 9.378 & - & 10.502 \\
\hline$q$ & 0.407 & 0.394 & 0.403 & 0.386 & 0.408 & 0.401 \\
\hline$\ell$ & 0.057 & 0.136 & 0.065 & 0.169 & 0.000 & 0.022 \\
\hline$k$ & 0.042 & 0.059 & 0.042 & 0.060 & 0.042 & 0.058 \\
\hline$R_{I}-(1-\delta) R_{D}$ & 3.239 & 3.294 & 3.205 & 3.280 & 3.163 & 3.183 \\
\hline$I+L$ & 0.914 & 0.965 & 0.933 & 0.997 & 0.906 & 0.928 \\
\hline$I-E-P O$ & 0.824 & 0.777 & 0.834 & 0.769 & 0.867 & 0.855 \\
\hline$E(D i v)$ & 0.745 & 0.789 & 0.747 & 0.796 & 0.743 & 0.779 \\
\hline$\Delta \mathbb{U}_{E}$ & - & - & $0.33 \%$ & $-0.62 \%$ & $1.19 \%$ & $1.93 \%$ \\
\hline$\Delta \mathbb{U}_{S}$ & - & - & $0.71 \%$ & $2.55 \%$ & $-0.30 \%$ & $-1.34 \%$ \\
\hline$\Delta \mathbb{U}_{B}$ & - & - & $-0.05 \%$ & $0.59 \%$ & $-0.09 \%$ & $-0.19 \%$ \\
\hline$\Delta \mathbb{U}_{O}$ & - & - & - & $0.36 \%$ & - & $-0.05 \%$ \\
\hline$\Delta \mathbb{U}_{s p}$ & & & $0.05 \%$ & $0.06 \%$ & $0.14 \%$ & $0.19 \%$ \\
\hline
\end{tabular}

Table D.7: Privately versus Socially optimal solutions when the bank issues outside equity $(O E)$ and $w_{O}=0$. The welfare changes are computed over the levels of welfare in the respective private equilibrium, which are normalized to one. We have set $e_{O}=0.09$ such that outside investors are willing to buy equity at the price offered by the bank, which is true as long as $e_{O}>0.062$.

\section{D.8 Distorted Deposit Supply and Loan Demand Schedules}

This section extends the analysis in section 3 by allowing the planner to use tools to distort the deposit supply and loan demand schedules of savers and entrepreneurs. We consider generic tools, $\tau_{D S}$ for the deposit supply schedule and $\tau_{L D}$ for the loan demand schedule, and discuss how they can be implemented in practice.

The deposit supply schedule (2) that the planner faces becomes:

$$
\begin{aligned}
U^{\prime}\left(e_{S}-D-L_{S}\right) & =\left[\beta \delta+\beta^{2}(1-\delta)\right] r_{D} \int_{\underline{\xi}}^{\xi^{*}} \theta(\xi, 1) \frac{d \xi}{\Delta_{\xi}} \\
& +\left[\beta_{D}+\beta^{2}(1-\delta)_{D}+V^{\prime}\left(D r_{D}\right) r_{D}\right](1-q)+\tau_{D S}
\end{aligned}
$$

The planner can distort the willingness of savers to hold deposits at given deposit rates by 


\begin{tabular}{lrrrrr}
\hline \hline & PE & \multicolumn{4}{c}{ SP for weights $\left(w_{E}, w_{S}, w_{O}\right)$} \\
$I$ & & $(0.1,0.1,0.0)$ & $(0.2,0.0,0.0)$ & $(0.1,0.1,0.1)$ & $(0.2,0.0,0.1)$ \\
\cline { 2 - 4 } \cline { 5 - 6 }$L_{1}$ & 0.834 & 0.829 & 0.908 & 0.824 & 0.907 \\
$D$ & 0.131 & 0.168 & 0.020 & 0.179 & 0.023 \\
$E$ & 0.908 & 0.937 & 0.875 & 0.942 & 0.876 \\
$O$ & 0.041 & 0.043 & 0.039 & 0.043 & 0.039 \\
$E /(E+O)$ & 0.002 & 0.002 & 0.001 & 0.002 & 0.001 \\
$R_{I}$ & 0.962 & 0.959 & 0.965 & 0.956 & 0.963 \\
$R_{D}$ & 4.142 & 4.154 & 4.047 & 4.161 & 4.048 \\
$P$ & 1.697 & 1.748 & 1.727 & 1.749 & 1.726 \\
$q$ & 9.902 & 9.378 & 10.502 & 9.200 & 10.392 \\
$\ell$ & 0.394 & 0.386 & 0.401 & 0.385 & 0.401 \\
$k$ & 0.136 & 0.169 & 0.022 & 0.178 & 0.024 \\
$R_{I}-(1-\delta) R_{D}$ & 0.059 & 0.060 & 0.058 & 0.061 & 0.058 \\
$I+L$ & 3.294 & 3.280 & 3.183 & 3.287 & 3.185 \\
$I-E-P O$ & 0.965 & 0.997 & 0.928 & 1.003 & 0.930 \\
$E($ Div $)$ & 0.777 & 0.769 & 0.855 & 0.763 & 0.853 \\
$\Delta \mathbb{U}_{E}$ & 0.789 & 0.796 & 0.779 & 0.800 & 0.781 \\
$\Delta \mathbb{U}_{S}$ & - & $-0.06 \%$ & $1.93 \%$ & $-0.17 \%$ & $1.91 \%$ \\
$\Delta \mathbb{U}_{B}$ & - & $1.22 \%$ & $-1.34 \%$ & $1.41 \%$ & $-1.30 \%$ \\
$\Delta \mathbb{U}_{O}$ & - & $-0.06 \%$ & $-0.19 \%$ & $-0.07 \%$ & $-0.19 \%$ \\
\hline \hline & - & $0.05 \%$ & $-0.05 \%$ & $0.10 \%$ & $-0.02 \%$ \\
\hline
\end{tabular}

Table D.8: Privately versus Socially optimal solutions when the bank issues outside equity. The welfare changes are computed over the levels of welfare in the private equilibrium, which are normalized to one. We have set $e_{O}=0.09$ such that outside investors are willing to buy equity at the price offered by the bank, which is true as long as $e_{O}>0.062$.

varying the level of the distortionary tool $\tau_{D S}$. In other words, the planner can set $\tau_{D S}$, which implies that (D.37) stops being a constraint in her optimization problem defined in Definition 2 and, thus, $\zeta_{D S}=0$ in (43). The intervention can be implemented, for example, either as a tax on the supply of deposits at $t=1$ or as a tax on the interest income accruing to late depositors at $t=3$ when the bank is solvent. In the first case, the tax can be computed as $-\tau_{D S} / U^{\prime}\left(e_{R}-D-L_{S}\right)$, while in the second, as $-\tau_{D S} /\left(\beta^{2}(1-\delta) \omega R_{D}(1-q)\right)$. If $\tau_{D S}<0$, then a tax is levied, while $\tau_{D S}>0$ implies a subsidy. We assume that the planner rebates the tax proceeds back to the same agents in the same period in a lump-sum fashion in order to neutralize any income effects.

Similarly, the loan demand schedule (7) becomes:

$$
\int_{\xi^{*}}^{\bar{\xi}}\left\{\omega\left[A-R_{I}\right](1-y(\xi, \delta))-c^{\prime}(I)\right\} \frac{d \xi}{\Delta_{\xi}}+\tau_{L D}=0 .
$$

The planner can distort the willingness of entrepreneurs to borrow by varying the level of the distortionary tool $\tau_{L D}$, such that, if $\tau_{L D} \neq 0$, then $\zeta_{L D}=0$ in (43). The intervention can be imple- 
mented with a tax on loan repayment in the good state of the world, which can be computed as $-\tau_{L D} /\left(\int_{\xi^{*}}^{\bar{\xi}}(1-y(\xi, \delta)) R_{I} d \xi / \Delta_{\xi}\right)$. If $\tau_{L D}<0$, then a tax is levied, while $\tau_{L D}>0$ implies a subsidy.

Given that the planner may choose to distort the deposit supply and loan demand schedules, we cannot use the indirect utilities (4) and (8), which imply the social welfare function (38). Instead, the planner maximizes the more elaborate social welfare function (37), which considers the direct utilities (1) and (5).

Table D.9 reports the planning equilibria under two sets of weights and three configurations: the benchmark one where the planner respects the deposit supply and loan demand schedule, a second where she distorts the deposit supply, and a third where she distorts the loan demand. When the planner can distort the deposit supply schedule, she can convince savers to supply deposits even if this is not optimal for them. For example, a distortionary subsidy on deposits makes savers want to supply more deposits and accept lower deposit rates, which is beneficial for the banker and entrepreneurs: lower deposit rates increase the profit margin of the banker, who is then willing to extend more loans at a lower loan rate. The planner goes all the way down to extracting all the surplus from depositors and pushing them to their participation constraint, i.e., their utility in autarky. ${ }^{10}$ The opposite is true when the planner distorts the loan demand schedule, i.e., $\tau_{L D} \neq 0$. A distortionary subsidy on investment/borrowing to $E$ allows the planner to increase lending without having to attract $E$ by offering a lower loan rate. Higher lending requires more deposits, which pushes deposit rates up and enhances transaction services. Yet, the planner can compensate the banker with a higher loan rate, which provides incentives for injecting more equity. The planner goes all the way to extracting all the surplus from entrepreneurs and pushing them to their participation constraint.

The higher the weight on entrepreneurs is, the higher is their utility under a positive $\tau_{D S}$, which distorts the deposit supply schedule urging savers to supply more deposits for lower deposit rates. Similarly, the higher the weight on savers is, the higher is their utility under a positive $\tau_{L D}$, which distorts the loan demand schedule urging entrepreneurs to borrow more for higher loan rates. In all cases, the planner still cares about the banker and can transfer some of the surplus back to her. As the weight on other agents becomes higher, this transfer will become smaller. Note that this was not possible in our benchmark analysis because both the banker and the planner had to respect the deposit supply and loan demand schedules. Hence, the banker was always losing from the planner's interventions.

\footnotetext{
${ }^{10}$ Given that the planner distorts the deposit supply schedule (2), savers' welfare in equilibrium is not given by (4) but rather (1). Hence, there is no guarantee that savers are strictly better off in the banking equilibrium compared to autarky (see footnote 4$)$.
} 


\begin{tabular}{|c|c|c|c|c|c|c|c|}
\hline & \multirow[t]{2}{*}{$\mathrm{PE}$} & \multicolumn{3}{|c|}{ SP for $\left(w_{E}, w_{S}\right)=(0.05,0.15)$} & \multicolumn{3}{|c|}{ SP for $\left(w_{E}, w_{S}\right)=(0.15,0.05)$} \\
\hline & & No tools & $\tau_{D S} \neq 0$ & $\tau_{L D} \neq 0$ & No tools & $\tau_{D S} \neq 0$ & $\tau_{L D} \neq 0$ \\
\hline$I$ & 0.862 & 0.841 & 1.190 & 1.002 & 0.899 & 1.211 & 0.991 \\
\hline$L$ & 0.052 & 0.119 & 0.000 & 0.000 & 0.012 & 0.000 & 0.000 \\
\hline$D$ & 0.875 & 0.919 & 1.164 & 0.956 & 0.873 & 1.184 & 0.946 \\
\hline E & 0.038 & 0.041 & 0.026 & 0.045 & 0.038 & 0.027 & 0.045 \\
\hline$R_{I}$ & 4.097 & 4.131 & 3.706 & 4.312 & 4.051 & 3.678 & 4.316 \\
\hline$R_{D}$ & 1.717 & 1.778 & 1.668 & 2.047 & 1.761 & 1.689 & 2.008 \\
\hline$q$ & 0.407 & 0.398 & 0.422 & 0.392 & 0.407 & 0.420 & 0.393 \\
\hline$\ell$ & 0.057 & 0.124 & 0.000 & 0.000 & 0.013 & 0.000 & 0.000 \\
\hline$k$ & 0.042 & 0.043 & 0.022 & 0.045 & 0.042 & 0.022 & 0.046 \\
\hline$R_{I}-(1-\delta) R_{D}$ & 3.239 & 3.242 & 2.872 & 3.288 & 3.170 & 2.833 & 3.312 \\
\hline$I+L$ & 0.914 & 0.960 & 1.190 & 1.002 & 0.911 & 1.211 & 0.991 \\
\hline$I-E$ & 0.824 & 0.800 & 1.164 & 0.956 & 0.861 & 1.184 & 0.946 \\
\hline$E(D i v)$ & 0.745 & 0.750 & 0.858 & 0.864 & 0.743 & 0.859 & 0.864 \\
\hline$\Delta \mathbb{U}_{E}$ & 0.076 & $-0.44 \%$ & $11.87 \%$ & $-7.61 \%$ & $1.02 \%$ & $12.97 \%$ & $-7.61 \%$ \\
\hline$\Delta \mathbb{U}_{S}$ & 1.050 & $1.74 \%$ & $-4.34 \%$ & $3.35 \%$ & $-0.10 \%$ & $-4.34 \%$ & $2.87 \%$ \\
\hline$\Delta \mathbb{U}_{B}$ & 0.327 & $-0.13 \%$ & $14.13 \%$ & $10.11 \%$ & $-0.08 \%$ & $14.02 \%$ & $10.16 \%$ \\
\hline$\tau_{D S}$ & - & - & 0.164 & - & - & 0.173 & - \\
\hline$\tau_{L D}$ & - & - & - & 0.122 & - & - & 0.120 \\
\hline
\end{tabular}

Table D.9: Privately versus Socially Optimal Solutions when additional distortionary tools are available. The welfare changes are computed over the level of welfare in the private equilibrium, which is normalized to one for each agent. 